HUGO DANIEL HERNANDEZ HERRERA

NOISE AND PSRR IMPROVEMENT TECHNIQUE FOR TPC READOUT FRONT-END IN CMOS TECHNOLOGY

São Paulo 
HUGO DANIEL HERNÁNDEZ HERRERA

\section{NOISE AND PSRR IMPROVEMENT TECHNIQUE FOR TPC READOUT FRONT-END IN CMOS TECHNOLOGY}

Tese apresentada à Escola

Politécnica da Universidade de

São Paulo para obtenção do

Título de Doutor em Ciências

Área de concentração:

Microeletrônica.

Orientador:

Prof. Dr Wilhelmus A. M. Van Noije

São Paulo 
Este exemplar foi revisado e corrigido em relação à versão original, sob responsabilidade única do autor e com a anuência de seu orientador.

São Paulo, de de

Assinatura do autor:

Assinatura do orientador:

HERRERA, HUGO DANIEL

NOISE AND PSRR IMPROVEMENT TECHNIQUE FOR TPC READOUT FRONT-END IN CMOS TECHNOLOGY / H. D. HERRERA -- versão corr. -São Paulo, 2016.

$101 \mathrm{p}$.

Tese (Doutorado) - Escola Politécnica da Universidade de São Paulo. Departamento de Engenharia de Sistemas Eletrônicos.

1.Readout front-end 2.Time Projection Chamber 3.Integrated Circuits 4.CMOS I.Universidade de São Paulo. Escola Politécnica. Departamento de Engenharia de Sistemas Eletrônicos II.t. 
A presente tese é dedicada a Deus por me dar saúde e força para realizar este trabalho.

A meu amado filho Juan Daniel

e a minha adorada esposa Elisa, vôces são minha luz. A mi amada madre, que tanto me estimuló desde niño para ser el hombre que soy hoy.

A toda mi familia. 


\section{AGRADECIMENTOS}

Agradeço a meu orientador, Dr. Wilhelmus A. M. Van Noije, pelos seus valiosos conselhos e pela oportunidade de desenvolver este trabalho na Universidade de São Paulo. Também ao professores Dr. Marcelo Munhoz e Dr. Marco Bregant por me dar a oportunidade de participar no projeto SAMPA e me apoiar em todo momento.

À os pesquisadores do CERN Alex Kluge, Marek Idzik, Hans Kristian Soltveit, Britton Jr Charles L and Marek Idzik, obrigado pelas tantas sugestões para este trabalho.

À fundação de Amparo e Pesquisa do Estado de São Paulo (FAPESP) que me outorgou a bolsa de estudo e financiou a fabricação dos circuitos integrados para a relização deste trabalho.

Ao Laboratório de Sistemas Integráveis (LSI) por facilitar suas infra-estruturas físicas.

Ao pessoal do DMPSV, colombianos e brasileiros por sua ajuda neste trabalho. 


\section{RESUMO}

ALICE é um dos quatro grandes experimentos do acelerador de partículas LHC (Large Hadron Collider) instalado no laboratório europeu CERN. Um programa de atualizações desse experimento acaba de ser aprovado pelo comitê gestor do acelerador LHC. Dentro das atualizações planejadas para os próximos anos do experimento ALICE, está melhorar a resolução e eficiência de rastreamento de partículas produzidas em colisões entre íons pesados, mantendo a excelente capacidade de identificação de partículas para uma taxa de leitura de eventos significativamente maior da atual. Para se alcançar esse objetivo, entre outras ações, é preciso atualizar os detectores Time Projection Chamber (TPC), modificando a eletrônica de leitura de eventos, a qual não é adequada para esta migração.

Para superar esta limitação tem sido proposto o projeto, simulação, fabricação, teste experimental e validação de um ASIC protótipo de aquisição de sinais e de processamento digital chamado SAMPA, que possa ser usado na eletrônica de detecção dos sinais no cátodo do TPC, que suporte polaridades negativas de tensão de entrada e leitura continua de dados, com 32 canais por chip, com menor consumo de potência comparado com a versão anterior do chip.

Este trabalho tem como objetivo o projeto, fabricação, e teste experimental de um readout front-end em tecnologia CMOS $130 \mathrm{~nm}$, com polaridade configurable (positiva/negativa), peaking time e sensibilidade, de forma que o novo SAMPA ASIC possa ser usada em ambos detectores. Para obter um ASIC integrando 32 canais por chip, o projeto do front-end proposto precisa ter baixa área e baixo consumo de potência, mas ao mesmo tempo requer baixo ruido. Neste sentido, uma nova técnica para melhorar a especificação de ruido e o PSRR (Power Supply Rejection Ratio) sem impacto no consumo de área e potência é proposta neste trabalho. A análise e as equações do circuito proposto são apresentadas as quais foram validadas por simulação e teste experimental de um circuito integrado com 5 canais do front-end projetado. O Equivalent Noise Charge medido foi $<550$ e para uma capacitance do detector de $18.5 \mathrm{pF}$. A área total do front-end foi de $2300 \mu m \times 150 \mu m$, e o consumo total de potencia medido foi de $9.1 \mathrm{~mW}$ por canal. 


\begin{abstract}
ALICE is one of four major experiments of particle accelerator LHC installed in the European laboratory CERN. The management committee of the LHC accelerator has just approved a program update for this experiment. Among the upgrades planned for the coming years of the ALICE experiment is to improve the resolution and tracking efficiency maintaining the excellent particles identification ability, and to increase the read-out event rate to $100 \mathrm{KHz}$. In order to achieve this, it is necessary to update the Time Projection Chamber detector (TPC) and Muon tracking (MCH) detector modifying the read-out electronics, which is not suitable for this migration.

To overcome this limitation the design, fabrication and experimental test of new ASIC named SAMPA has been proposed. This ASIC will support both positive and negative polarities, with 32 channels per chip and continuous data readout with smaller power consumption than the previous versions.

This work aims to design, fabrication and experimental test of a readout front-end in 130nm CMOS technology with configurable polarity (positive/negative), peaking time and sensitivity. The new SAMPA ASIC can be used in both chambers (TPC and MCH). The proposed front-end is composed of a Charge Sensitive Amplifier (CSA) and a Semi-Gaussian shaper. In order to obtain an ASIC integrating 32 channels per chip, the design of the proposed front-end requires small area and low power consumption, but at the same time requires low noise. In this sense, a new Noise and PSRR (Power Supply Rejection Ratio) improvement technique for the CSA design without power and area impact is proposed in this work. The analysis and equations of the proposed circuit are presented which were verified by electrical simulations and experimental test of a produced chip with 5 channels of the designed front-end. The measured equivalent noise charge was $<550$ e for $30 \mathrm{mV} / \mathrm{fC}$ of sensitivity at a input capacitance of $18.5 \mathrm{pF}$. The total core area of the front-end was $2300 \mu \mathrm{m} \times 150 \mu \mathrm{m}$, and the measured total power consumption was $9.1 \mathrm{~mW}$ per channel.
\end{abstract}




\section{Contents}

1 Introduction 1

1.1 ALICE TPC . . . . . . . . . . . . . . . . . . 3

1.2 TPC signal amplification . . . . . . . . . . . . . . . 4

$1.3 \mathrm{TPC}$ read-out electronic . . . . . . . . . . . . . . . 5

1.4 MWPC limitations . . . . . . . . . . . . . . . . . 6

1.5 ALICE update . . . . . . . . . . . . . . . . . 7

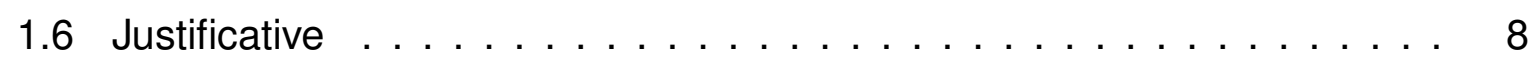

1.7 Objectives . . . . . . . . . . . . . . . . . 10

1.8 Contributions of this thesis $\ldots \ldots \ldots \ldots \ldots$

1.9 Thesis summary $\ldots \ldots \ldots \ldots \ldots$. . . . . . . . . . . . . . . . . . . . . .

2 Readout front-end for gaseous detectors 13

2.1 Readout front-end specifications . . . . . . . . . . . . . . 15

2.1 .1 Sensitivity . . . . . . . . . . . . . . . . 15

2.1 .2 Pulse time specifications . . . . . . . . . . . 16

2.1 .3 Crosstalk . . . . . . . . . . . . . . . . 16

2.1 .4 Equivalent Noise Charge . . . . . . . . . . . . . . . . . . 17

2.1 .5 Input dynamic range . . . . . . . . . . . . . . . 17

2.1 .6 Non-Linearity . . . . . . . . . . . . . . . . . . . . 18

2.1.7 Pole-zero cancellation . . . . . . . . . . . . . . . . . . . . 18

2.2 Read-out front-end of the ALICE TPC . . . . . . . . . . . . . . . . 20

2.2 .1 PASA ASIC . . . . . . . . . . . . . . . . . . 20

2.2 .2 ALTRO ASIC . . . . . . . . . . . . . . . . . . . . . 21

2.3 New front-end ASIC: SAMPA . . . . . . . . . . . . . . . . . . . 23

2.3.1 SAMPA building blocks . . . . . . . . . . . . . 24 
3 Charge Sensitive Amplifier 26

3.1 CSA architecture . . . . . . . . . . . . . . . . 26

3.2 Design requirements of the CSA . . . . . . . . . . . . 27

3.2 .1 Open loop gain . . . . . . . . . . . . . . . . . . 27

3.2 .2 Residual charge . . . . . . . . . . . . . . . 28

3.2.3 Input impedance . . . . . . . . . . . . . . . . . . . 28

3.2 .4 Frequency response . . . . . . . . . . . . . . . . . . . . . . . 29

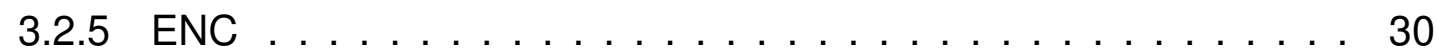

3.2.6 Rise time of the impulse response . . . . . . . . . . . . . 36

3.3 CSA Design . . . . . . . . . . . . . . . . . . 37

3.3.1 Feedback capacitance selection . . . . . . . . . . . . . . . 37

3.3 .2 GBW selection . . . . . . . . . . . . . . . . . . 38

3.3.3 Input transistor design . . . . . . . . . . . . . . . . . 39

3.3.4 Self-adaptive bias circuit . . . . . . . . . . . . . . . 45

3.3.5 Schematic of the CSA . . . . . . . . . . . . . . 47

3.4 Semi-Gaussian pulse shaper . . . . . . . . . . . . . . . . . . 49

4 Noise and PSRR improvement technique 54

4.1 PMOS-NMOS input transistor topology . . . . . . . . . . . . . . 56

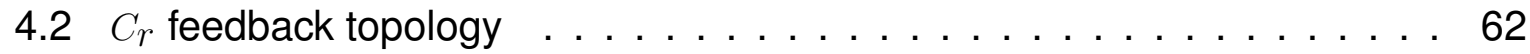

4.3 Front-end PSRR improvement . . . . . . . . . . . . . 65

4.3.1 Front-end PSRR . . . . . . . . . . . . . . . . . . . . . 65

4.3.2 PSRR of the CSA original topology . . . . . . . . . . . . . 66

4.3.3 PSRR of the PMOS-NMOS input transistor topology . . . . . . . . 71

4.3.4 PSRR of the $C_{r}$ feedback topology . . . . . . . . . . . . . . 74

5 Experimental results $\quad 78$

5.1 Description of the SAMPA chip01 . . . . . . . . . . . . . 79

5.2 Experimental Set-up of the SAMPA chip01 test . . . . . . . . . . . 81

5.2.1 DC Polarization and Power consumption . . . . . . . . . . . . . 83

5.2 .2 Output pulse specifications . . . . . . . . . . . . . . . 83

5.2 .3 Noise $($ ENC) $\ldots \ldots \ldots$. . . . . . . . . . . . . . . . . . . 91

5.2 .4 Cross-talk . . . . . . . . . . . . . . . . . . 92 
5.2 .5 PSRR of the Front-end . . . . . . . . . . . . . . . . . . . 94

6 Conclusions and Future Work 96 


\section{Nomenclature}

$\beta \quad$ MOS transistor gain factor

$\mu_{n} \quad$ Carrier mobility

$\phi_{t} \quad$ Thermal voltage

$\tau_{0} \quad$ time constant of the differentiator and integrator

$\tau_{s} \quad$ Peaking Time

$C_{f} \quad$ Feedback Capacitance of the CSA

$C_{\text {diff }}$ Capacitor of the differentiator circuit

$C_{g b} \quad$ Gate-bulk Capacitance

$C_{g s} \quad$ Gate-source capacitance

$C_{O X} \quad$ Gate oxide capacitance

ENC Equivalent Noise charge

f $\quad$ Frequency

$g_{m} \quad$ Transconductance

$G_{O} \quad$ Output conductance

$I_{d} \quad$ Drain current

Iz $\quad$ Characteristic current

$K_{f} \quad$ Flicker noise coefficient

$n \quad$ Number of integrators of the Semi-gaussiam Shaper 
$n_{s} \quad$ Number ofthe single poly-stripe

$P S R R$ Power supply rejection ratio

$R_{f} \quad$ Feedback Resistance of the CSA

$R_{g} \quad$ Gate resistance

$R_{i} \quad$ Resistance of the single poly-stripe

$R_{O} \quad$ Output Resistance

$R_{p z} \quad$ Resistor of the pole-zero cancellation network

$S_{f} \quad$ Power spectral densities of the flicker noise

$S_{i} \quad$ Power spectral densities of the channel thermal noise

$t_{p z} \quad$ Time constant of the pole-zero cancellation network

$V_{t} \quad$ Threshold voltage

$V_{g s} \quad$ Gate-source voltage

$V_{\text {out }}$ Output Voltage of the CSA

$Z_{i} \quad$ Input Impedance

A DC gain of the CSA

ALICE A Large Ion Collider Experiment

ASIC Applications Specific Integrated Circuit

CERN European Organization for Nuclear Research

CSA Charge Sensitive Amplifier

DAQ Data AcQuisition

DCS Detector Control System

ESD Electrostatic discharge

$\mathrm{f}(\mathrm{u})$ Interpolation function 
FECs Front-End Cards

FWHM Full Width at Half Maximum

GaAS Gallium Arsenide

GBW Gain-bandwidth product

GEM Gas Electron Multiplier

INL Integrated non-Linearity

INL Integrated non-Linearity

LHC Large Hadron Collider

MPGD Micro-Pattern Gas Detector

MWPC Multi-Wire Proportional Chambers

Q Input charge injected on the CSA input

$\mathrm{R}_{\text {esd }}$ Resistence of the ESD protection

RCU Readout Control Unit

TPC Time Projection Chamber

u Inversion coefficient 


\section{List of Figures}

1.1 Overview of $\mathrm{LHC}$ and its experiments $\ldots \ldots \ldots \ldots \ldots \ldots$

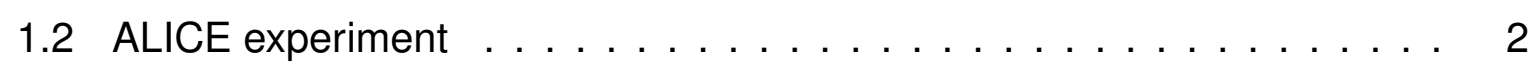

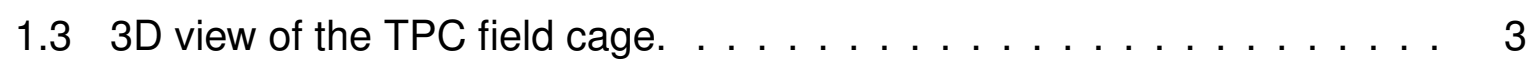

1.4 Schematic drawing for a simplistic gaseous radiation detector . . . . . . 4

1.5 MWPC schematic technology of the ALICE TPC . . . . . . . 5

1.6 An overview of the ALICE TPC front end electronics . . . . . . . 6

1.7 GEM structure diagram taken from CERN Gas Detector Development group $[$ CERN... $] \ldots \ldots \ldots \ldots \ldots \ldots$

2.1 Typical block diagram of the electronic read-out system of a gaseous

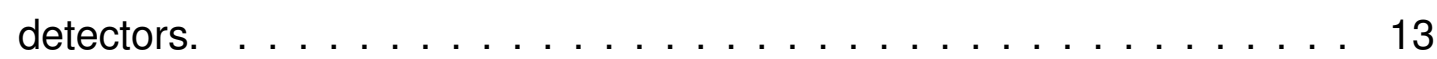

2.2 (a) A semi-Gaussian pulse waveforms with normalized amplitude for 160ns of peaking time and $n=2,4,6$; (b) pulse waveform variation for 40ns, $80 \mathrm{~ns}$ and $120 \mathrm{~ns}$ of peaking time $(n=6)$ with normalized amplitude. 15

2.3 FWHN, shaping time and peaking time specifications of a semi-Gaussian

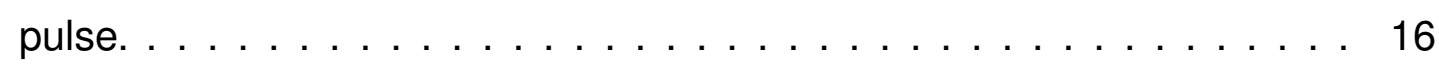

2.4 Pulse coupling between adjacent channels for a 5 channels front-end

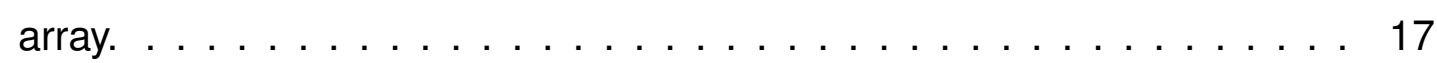

2.5 (a) Input dynamic range definition; (b) Integrated non-Linearity (INL)

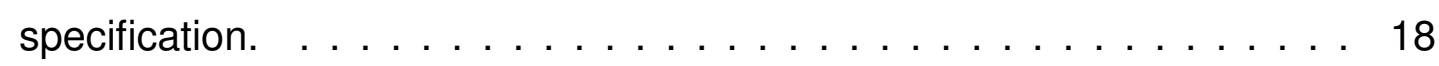

2.6 Diagram of the pole-zero cancellation circuit. . . . . . . . . . . 19

2.7 Simplified block diagram of the signal processing chain of the PASA channel. Figure taken from [SOLTVEIT et al., 2012] . . . . . . . . 21

2.8 Block diagram of the ALTRO chip. . . . . . . . . . . . . . . 22

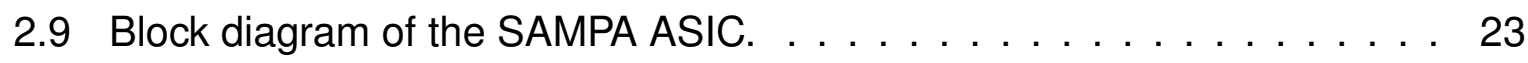


3.1 Selected topology for the amplifier of the CSA based on a single-ended folded cascode.

3.2 (a) Simple model of the CSA for the CSA open loop gain estimation; (b) Dominant pole model of the CSA; (c) Circuit equivalent to estimate the crosstalk between adjacent channels.

3.3 Crosstalk versus frequency obtained from equation 3.9 for $A=40 \mathrm{~dB}, \mathrm{GBW}=2 \mathrm{GHz}$,

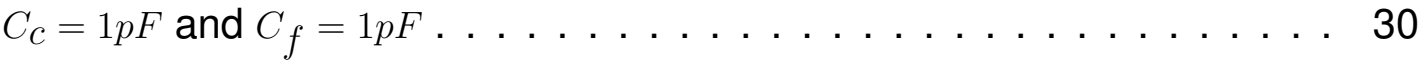

3.4 Front-end noise model for a Semi-Gaussian shaper. . . . . . . . . . 31

3.5 Equivalent circuit for noise analysis of the bias current mirror. . . . . . 34

3.6 Block schematic of the CSA transient model . . . . . . . . . . 36

3.7 Small signal model of the cascode amplifier for the open loop gain esti-

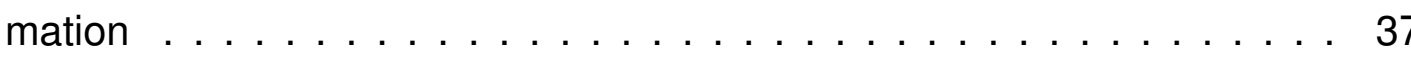

3.8 Theoretical ENC components versus the channel width (W) of the CSA input transistor for $I_{d}=1 \mathrm{~mA}, L=130 \mathrm{~nm}$ and (a) $t_{s}=160 \mathrm{~ns}, C_{d}=18.5 \mathrm{pF}$; (b) $t_{s}=80 \mathrm{~ns}, C_{d}=18.5 \mathrm{pF}$; (c) $t_{s}=300 \mathrm{~ns}, C_{d}=40 \mathrm{pF} \ldots \ldots \ldots \ldots \ldots$

3.9 Theoretical $E N C_{t}$ versus W for $I_{d}=250 u A, I_{d}=2 m A, I_{d}=3 m A$ and (a) $t_{s}=160 \mathrm{~ns}, C_{d}=18.5 p F$; (b) $t_{s}=80 \mathrm{~ns}, C_{d}=18.5 \mathrm{pF}$; (c) $t_{s}=300 \mathrm{~ns}$, $C_{d}=40 p F \ldots \ldots \ldots \ldots \ldots \ldots \ldots \ldots \ldots \ldots \ldots \ldots \ldots \ldots \ldots \ldots$

3.10 Theoretical $E N C_{t}$ versus $I_{d}$ for $t_{s}=160 n s, C_{d}=18.5 p F ; t_{s}=80 n s, C_{d}=$ $18.5 p F ; t_{s}=300 n s, C_{d}=40 p F \ldots \ldots \ldots \ldots \ldots \ldots \ldots$

3.11 Inversion factor $I_{d} / I_{z}$ as a function of $W\left(\mathrm{~L}=L_{\min }\right)$ and $I_{d}=2 \mathrm{~mA} . \ldots .44$

3.12 Transistor level schematic of the adaptive bias circuit. . . . . . . . 46

3.13 Transistor level schematic of the CSA . . . . . . . . . . . . . . 48

3.14 Layout of the designed CSA. . . . . . . . . . . . . . . 48

3.15 Block diagram including the CSA and the shaper first stage. . . . . . . 49

3.16 Transistor level schematic of: (a)Pulse shaper first stage; (b) Pulse shaper second stage $\ldots \ldots \ldots \ldots \ldots \ldots$

3.17 Transistor level schematic of: (a) Bias current generator; (b) Non-inverting

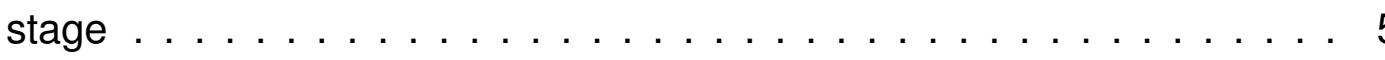

4.1 $\mathrm{ENC}_{M B 0}$ component in terms of the bias decoupling capacitance $\left(C_{B P}\right)$ simulation of the original NMOS input transistor topology with $C_{d}=$ $18.5 p F, t_{s}=160 \mathrm{~ns}, S e n=30 \mathrm{mV} / \mathrm{fC}$ and $R_{S}=0 \Omega . \ldots \ldots \ldots \ldots$ 
4.2 Schematic of the CSA with the proposed PMOs input transistor . . . . . . 56

4.3 Small signal equivalent circuit of the proposed topology adding a PMOS input transistor $\left(M_{2}\right) \ldots \ldots \ldots \ldots \ldots$

4.4 ENC components of $M_{B 0}, M_{B 2}$ and $M_{B 3}$ in relation to the detector capacitance for $t_{s}=160 \mathrm{~ns}, \operatorname{Sen}=30 \mathrm{mV} / \mathrm{fC}$ and $R_{S}=0 \Omega$ : (a) NMOS input transistor topology (b)PMOS-NMOS input transistor topology .

4.5 Comparison between the total ENC obtained with the original NMOS input transistor topology $\left(E N C_{N M O S}\right)$ and with the proposed PMOSNMOS input transistor topology $\left(E N C_{P N M O S}\right)$ in term of the detector capacitance for $C_{B P}=0 \mathrm{pF}, t_{s}=160 \mathrm{~ns}, S e n=30 \mathrm{mV} / \mathrm{fC}$ and $R_{s}=0 \Omega \ldots$. .

4.6 Total ENC in terms of the bias decoupling capacitance $\left(C_{B P}\right)$ of the original NMOS input transistor topology and with the proposed PMOS-NMOS input transistor topology $\left(E N C_{P N M O S}\right)$ for $C_{d}=18.5 p F, t_{s}=160 \mathrm{~ns}$, $S e n=30 \mathrm{mV} / \mathrm{fC}$ and $R_{s}=0 \Omega \ldots \ldots \ldots \ldots \ldots \ldots$. . . . . . . . . . . . . .

4.7 Total ENC generated by the designed bias current for $C_{d}=18.5 p F$, $t_{s}=160 \mathrm{~ns}$, Sen $=30 \mathrm{mV} / \mathrm{fC}$ and $R_{S}=0 \Omega$.

4.8 ENC simulation corners for the proposed topology (PNMOS) and the NMOS input transistor topology for $C_{d}=18.5 p F, t_{s}=160 \mathrm{~ns}, S e n=30 \mathrm{mV} / \mathrm{fC}$

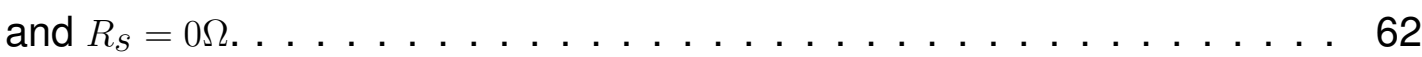

4.9 Schematic of the proposed $C_{r}$ feedback topology. . . . . . . . . . . . 62

4.10 Small signal equivalent circuit of the $C_{r}$ feedback topology. . . . . . . . 63

4.11 ENC components of $M_{B 0}, M_{B 2}$ and $M_{B 3}$ with $C_{r}$ feedback topology in relation to the detector capacitance for $t_{s}=160 \mathrm{~ns}, S e n=30 \mathrm{mV} / \mathrm{fC}$ and

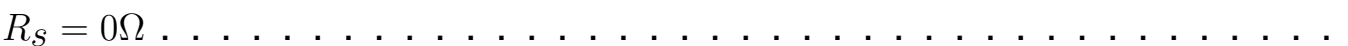

4.12 ENC simulation corners for the proposed $C_{r}$ feedback topology and the NMOS input transistor topology for $C_{d}=18.5 p F, t_{s}=160 \mathrm{~ns}, S e n=30 \mathrm{mV} / \mathrm{fC}$

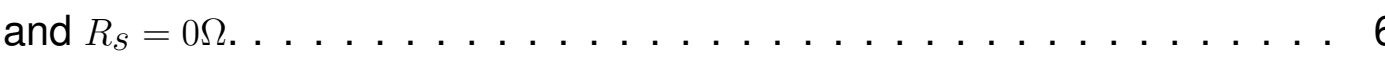

4.13 Simplified small signal model for the PSRR study if the CSA with the

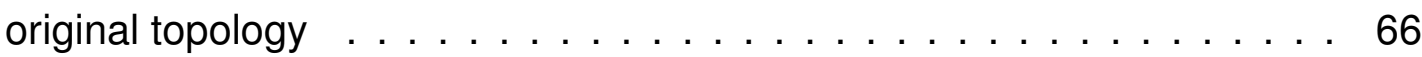

4.14 Theoretical PSRR from equation (4.24). . . . . . . . . . . . . . . . 68 
4.15 $\operatorname{PSRR}_{C S A}$ simulation of the designed CSA with the NMOS input transistor topology for $C_{d}=0 \mathrm{pF}-20 \mathrm{pF}-40 \mathrm{pF}, C_{f}=1.6 \mathrm{pF}, G_{f}=1 / 3 \mathrm{M} \Omega, g_{m 1}=$ $44 \mathrm{mS}, G_{0 B 2}=119 \mu S$ and $G_{0 B 2}=17 \mu S \ldots \ldots \ldots \ldots \ldots$

4.16 $P S R R_{C S A}$ at $2 \mathrm{MHz}$, simulation and the theoretical value versus the detector capacitance.

4.17 Simulation of the $P S R R_{F E}$ with the NMOS input transistor topology for $C_{d}=18.5 \mathrm{pF}, t_{s}=160 \mathrm{~ns}, S e n=30 \mathrm{mV} / \mathrm{fC}$ and $36 \mathrm{~dB}, 32.5 \mathrm{~dB}$ and $34 \mathrm{~dB}$ of front-end gain $\ldots \ldots \ldots \ldots \ldots \ldots \ldots \ldots \ldots \ldots$

4.18 Small signal model shown for the PSRR analysis of the PMOS-NMOS input transistor topology.

4.19 PSRR simulation of the designed CSA with PMOS-NMOS input transistor topology for $C_{d}=0 \mathrm{pF}-20 \mathrm{pF}-40 \mathrm{pF}, C_{f}=1.6 \mathrm{pF}, G_{f}=1 / 3 \mathrm{M} \Omega, g_{m 1}=$ $44 \mathrm{mS}, G_{0 B 2}=119 \mu S$ and $G_{0 B 2}=17 \mu S \ldots \ldots \ldots \ldots \ldots$

4.20 PSRR simulation and the theoretical PSRR at $2 \mathrm{MHz}$ versus the detector capacitance for the designed CSA with PMOS-NMOS input transistor topology.

4.21 Small signal model shown for the PSRR analysis of the $C_{r}$ feedback topology.

4.22 PSRR simulation of the designed CSA with $C_{r}$ feedback topology for $C_{d}=0 \mathrm{pF}-20 \mathrm{pF}-40 \mathrm{pF}, C_{f}=1.6 \mathrm{pF}, G_{f}=1 / 3 \mathrm{M} \Omega, g_{m 1}=44 \mathrm{mS}, G_{0 B 2}=119 \mu S$ and $G_{0 B 2}=17 \mu S \ldots \ldots \ldots \ldots \ldots \ldots \ldots \ldots \ldots \ldots \ldots \ldots \ldots \ldots$

4.23 Comparison of the PSRR at $2 \mathrm{MHz}$ of the designed CSA with the NMOS input transistor topology $\left(P S R R_{N M O S}\right)$, with the PMOS-NMOS input transistor topology and with the $C_{r}$ feedback topology for $t_{s}=160 \mathrm{~ns}$ and Sen $=30 \mathrm{mV} / \mathrm{fC}$. . . . . . . . . . . . . . . . . . . . . 77

5.1 Block diagram of the 5 front-end channels chip. . . . . . . . . . . 79

5.2 Layout of the 5 front-end channels chip. . . . . . . . . . . . . 79

5.3 Micro-photograph of the fabricated chip with 5 channels of the designed front-end in 130nm TSMC CMOS technlogy. . . . . . . . . . . . . 79

5.4 Photographic of the test board used for experimental testing of the packaged SAMPA chip $01 \ldots \ldots \ldots \ldots \ldots$. . . . . . . . . . 81

5.5 Test system set-up for the peaking time and sensitivity measurements. . 82 
5.6 Schematic of the resistive trimmable circuit used to generate the frontend voltage references. . . . . . . . . . . . . . . 83

5.7 Measured output pulses for different SAMPA chip01 configurations . . . 86

5.8 Chip01 measured analog output signals for a range of detector capacitances.

5.9 Amplifier output peak voltage $($ Vout $)$, linear fit $\left(\right.$ Vout $\left._{\text {fit }}\right)$ and $\mathrm{INL}[\%]$ as function of the input charge for the 3 sensitivity cases. . . . . . . . . 88

5.10 (a) Circuit sensitivity and (b) peaking time as a function of the detector capacitance for measurements (points) and simulation (straight line) for $4 \mathrm{mV} / \mathrm{fC}$ configuration.

5.11 (a) Circuit sensitivity and (b) peaking time as a function of the detector capacitance for measurements (points) and simulation (straight line) for $20 \mathrm{mV} / \mathrm{fC}$ configuration. . . . . . . . . . . . . . . . . . .

5.12 (a) Circuit sensitivity and (b) peaking time as a function of the detector capacitance for measurements (points) and simulation (straight line) for $30 \mathrm{mV} / \mathrm{fC}$ configuration. . . . . . . . . . . . . . . . .

5.13 (a) Circuit sensitivity and (b) peaking time measures as a function of the input charge for the $4 \mathrm{mV} / \mathrm{fC}$ configuration. . . . . . . . . . . . . 90

5.14 (a) Circuit sensitivity and (b) peaking time measures as a function of the input charge for the $20 \mathrm{mV} / \mathrm{fC}$ configuration. . . . . . . . . . . . . 90

5.15 (a) Circuit sensitivity and (b) peaking time measures as a function of the input charge for the $30 \mathrm{mV} / \mathrm{fC}$ configuration. . . . . . . . . . . . 9

5.16 Measured equivalent noise charge of the SAMPA chip01. The points show the measurements done for various chips and straight line the expected value from simulation for the $4 \mathrm{mV} / \mathrm{fC}$ configuration.

5.17 Measured equivalent noise charge of the SAMPA chip01. The points show the measurements done for various chips and straight line the expected value from simulation for the $20 \mathrm{mV} / \mathrm{fC}$ configuration. . . . . . . . . 92

5.18 Measured equivalent noise charge of the SAMPA chip01. The points show the measurements done for various chips and straight line the expected value from simulation for $30 \mathrm{mV} / \mathrm{fC}$ configuration. . . . . . . . . 9 
5.19 Experimental output parasitic pulse detected on channel 5 when channel

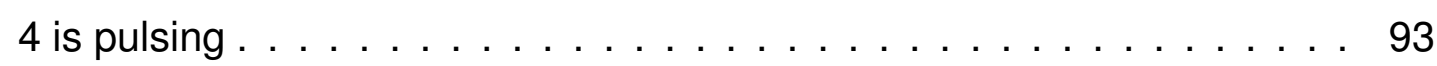

5.20 Experimental crosstalk specification on channel 5 and 3 in terms of the input charge when channel 4 is pulsing, for the Sen=30mV/fC@ $t_{s}=160 \mathrm{~ns}$ (a) and Sen=4mV/fC@ $t_{s}=300 n s$ (b) cases. . . . . . . . . . . . . 93

5.21 PSRR measurements for $20 \mathrm{mV} / \mathrm{fC}$ and $30 \mathrm{mV} / \mathrm{fC}$ configurations compared with the simulations results. . . . . . . . . . . . . . . . 95 


\section{List of Tables}

2.1 Specifications of the new front-end ASIC (SAMPA) . . . . . . . 25

3.1 Summary of the obtained GBW values according to the input impedance and rise time requirements . . . . . . . . . . . . 39

3.2 Continuous equations (model) valid in all regions of operation taken from [TSIVIDIS, 1987] . . . . . . . . . . . . . . . . . . . 40

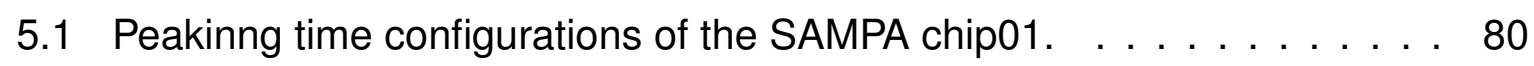

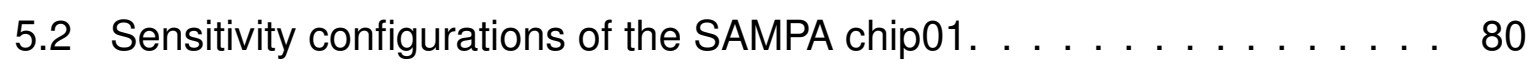

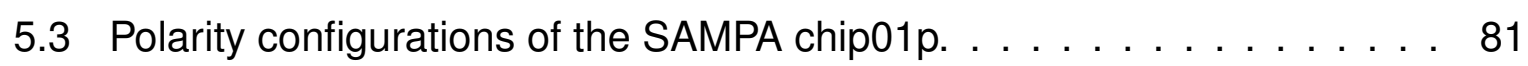

5.4 Technical characteristics of the equipments used in the experimental

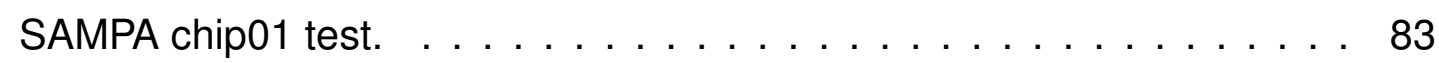




\section{Chapter 1}

\section{Introduction}

In a competitive and globalized world, the role of the basic sciences to social and technological development of nations have become increasingly important. Disciplines that seek to deepen the understanding of the nature and the universe, motivated by curiosity and the human need to understand the world around us, intensely stimulate the creation of new technologies. The investigation of fundamental nature aspects creates challenges that can only be overcome through the development of technology. An example of this symbiosis between basic science and technological innovation is the European laboratory CERN (European Organization for Nuclear Research). The CERN is a laboratory manged by 20 European nations with a mission to develop research in basic sciences, especially particle physics, technological applications, stimulate collaboration among scientists and create educational programs. With under 2.400 full-time employees and 1.500 part-time employees, the laboratoryhosts about 10.000 visiting scientists and engineers, representing 608 universities.

Most of the activities at CERN are currently directed towards operating the Large Hadron Collider (LHC). The LHC represents a large-scale, worldwide scientific cooperation project. The LHC is the world's largest and most powerful particle accelerator installed in the European laboratory CERN (European Organization for Nuclear Research). The LHC consists of a $27 \mathrm{Km}$ circular particle accelerator based on superconducting magnets with two opposite direction beams. It is located in Switzerland and France in a tunnel that is approximately $100 \mathrm{~m}$ deep underground. Four main experiments exploit the high-energy beams provided by the LHC, these are the ATLAS, CMS, ALICE and LHCb, as shown in the Figure1.1. 
The ALICE experiment (A Large Ion Collider Experiment) is a general-purpose heavy-ion experiment focused in studying the physics of strong interaction matter and the quark-luon plasma (QGP) in lead-lead collisions at the LHC. ALICE experiment is located at one of the collision points along the LHC, and targets primarily $\mathrm{Pb}-\mathrm{Pb}$ collisions. The ALICE is composed by almost two dozens of complementary detectors that allow the study of the properties of the particles that are created in the collisions. A system diagram of the ALICE experiment is shown in Figure 1.2. It has two distinct parts: the central barrel and the forward muon spectrometer. The ALICE Time Projection Chamber (TPC) is the main tracking detector of the central barrel [COLLABORATION et al., 2004]. The TPC is based on gaseous getectors principle: "When any type of radiation that is energetic enough to ionize the gas confined between the electrodes of different polarity passes through, emergent electrons and ions are drifted towards

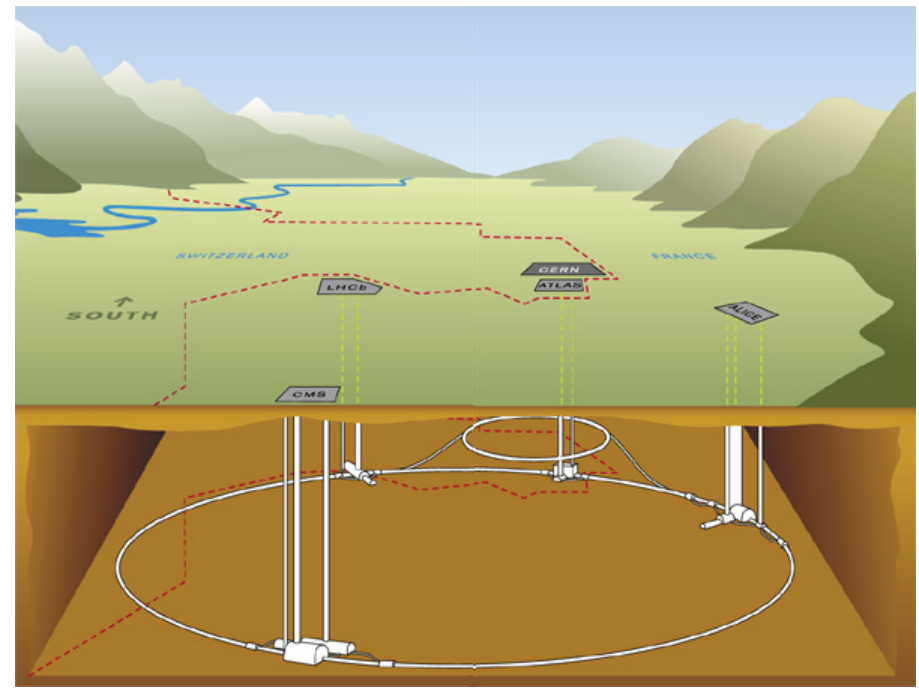

Figure 1.1: Overview of LHC and its experiments

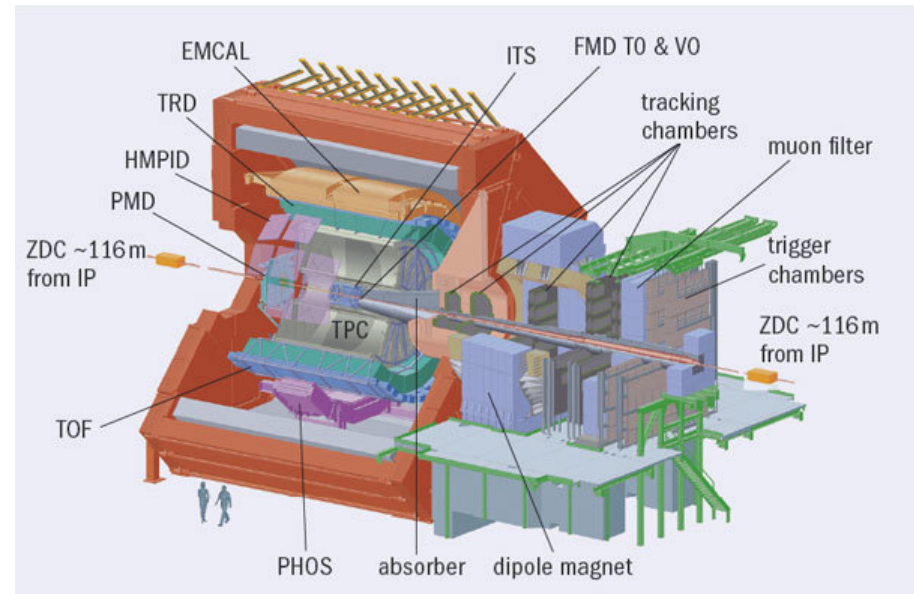

Figure 1.2: ALICE experiment 


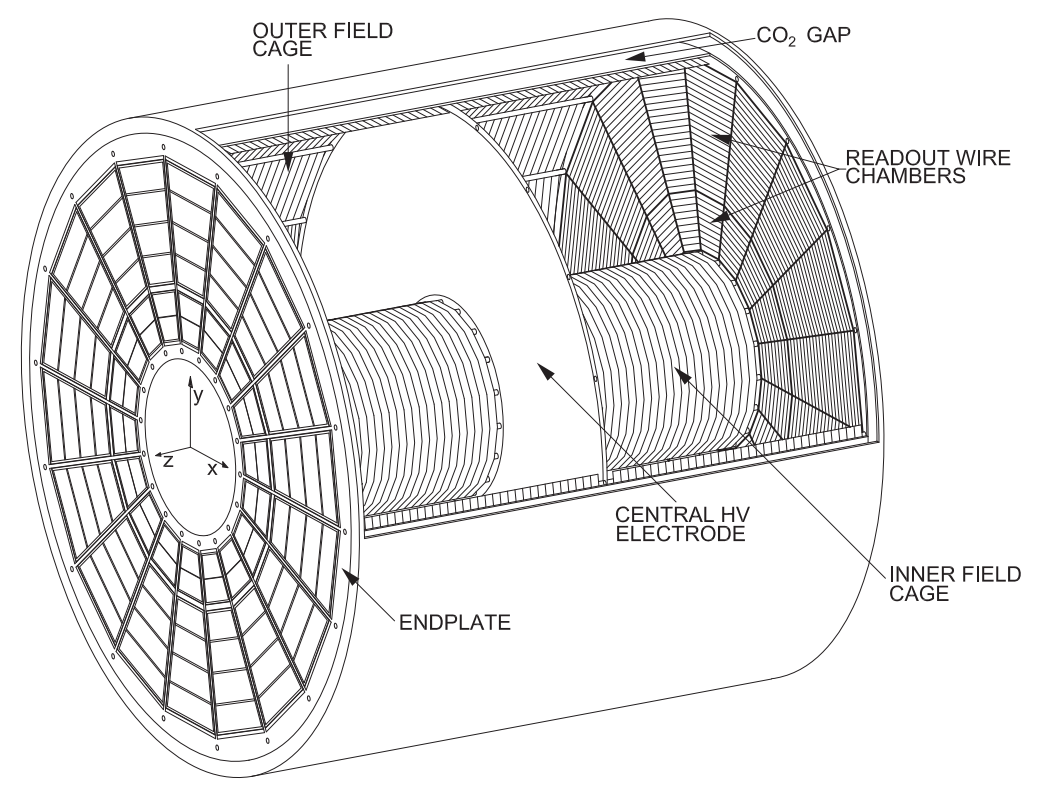

Figure 1.3: 3D view of the TPC field cage.

the anode and cathode respectively hence produce an electrical current" [BLUM et al., 2008]. Schematic drawing for a simplistic gaseous radiation detector is given in Figure 1.4 .

\subsection{ALICE TPC}

The ALICE TPC consists of a $90 \mathrm{~m}^{3}$ gas-filled $\left(\mathrm{Ne}-\mathrm{CO}_{2}\right)$ detection chamber in an electric field with a position-sensitive electron collection system. Along its length, the chamber is divided into halves by means of a central high-voltage electrode disc, which establishes an electric field between the center and the end plates. On passing through the detector gas, a particle will produce primary ionization along its track. The ionization trail that a crossing particle leaves on a gas can be used to detect its trajectory. If an electric field is applied to the gas volume, most of the free ions and electrons will not recombine but rather drift in opposite directions towards the high voltage and the endplates, where they can be sensed by the electronics after an amplification. The interpretation of the position in the plane where the electrons arrive gives a twodimensional track information of the crossing particle. Since the drift velocity of the electrons and ions is on average constant in the medium, the arrival time gives information about the distance between the ionization trail and the endplates. Therefore, a three dimensional trajectory reconstruction is possible with a two dimensional readout surface and the timing information [BLUM et al., 2008] [ALME et al., 2010]. 


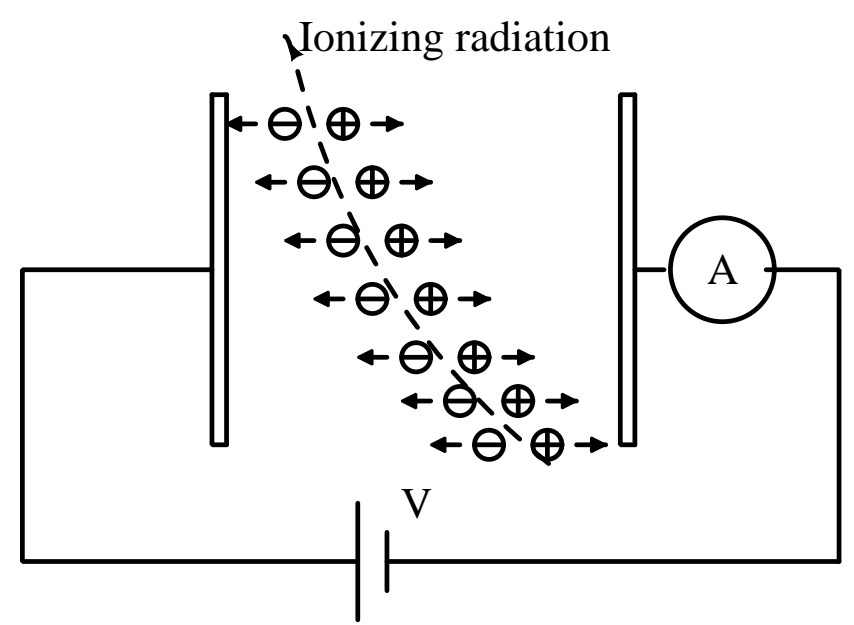

Figure 1.4: Schematic drawing for a simplistic gaseous radiation detector

\subsection{TPC signal amplification}

The number of electrons generated by the passage of the particles through the gas is too small to be sensed and acquired by the electronics. the ALICE TPC multiply the number of free electrons by about four orders of magnitude using the Multi-Wire Proportional Chambers (MWPC) technology. A MWPC schematic is ilustred in Figure 1.5. On the endplate inside the gas filled chamber a set of 3 staggered levels of wire grid named gating grid, cathode grid (or shielding grid) and anode grid (or sensing grid) generate the gas amplification. It presents two regions: the drift region or the effective volume where the trajectories of the crossing particles can be detected, and the amplification region where the ionization is amplified. A $100 \mathrm{kV}$ voltage between the anode (positive) and the catode grid (negative) is applied to produce the avalanche process that occurs at the vicinity of the anode wire grid. The anode grid accelerates the primary electrons generated from the crossing particle ionization with enough energy to produce multiple secondary gas ionizations. The read-out pads and the cathode grid collect the ions generated during the avalanche process but only the charge signal read-out pads is used in the trajectory reconstruction. A fraction of the generated ions in the avalanche can travel back towards the drift volume. The function of the gating grid is to collect these free ions avoiding that them enters into the drift volume and distorting the uniformity of the electric field [ALME et al., 2010]. 


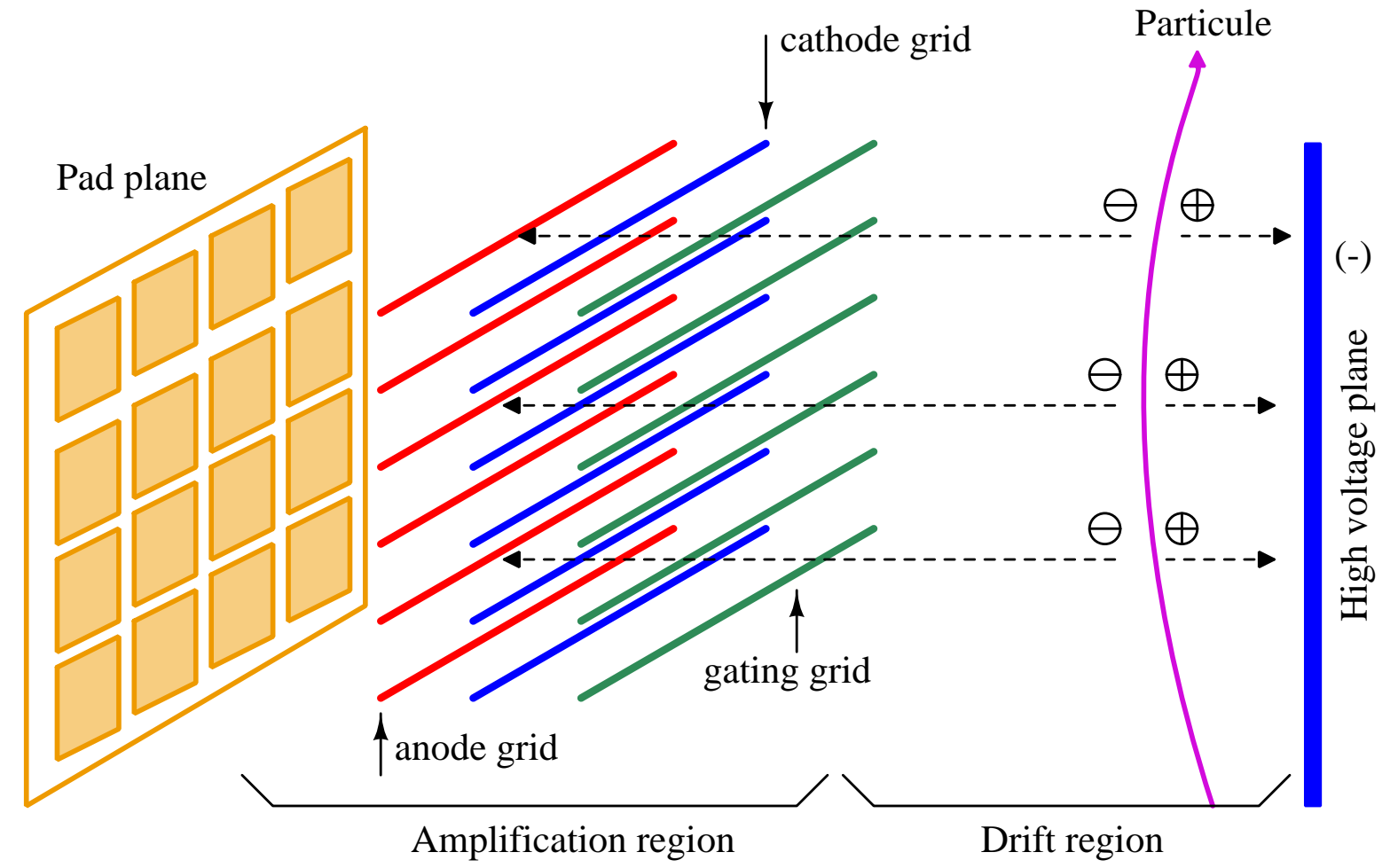

Figure 1.5: MWPC schematic technology of the ALICE TPC

\subsection{TPC read-out electronic}

A TPC read-out system diagram is ilustred in Figure 1.6 (taken from [MUSA, 2007]). Charged particles traversing the TPC volume ionize the gas along their path, liberating electrons that drift towards the endplate of the chamber. The signal amplification is provided through avalanche effect in the vicinity of the anode wires of the readout chambers. The electrons and positive ions created in the avalanche, which move, respectively, towards the anode wire and the surrounding electrodes, induce a positive current signal on the pad plane. The current signal of a single avalanche, which is characterized by a fast rise time $<1 \mathrm{~ns}$ ) and a long tail (of the order of $50 \mathrm{~ms}$ ), carries a charge that, in the ALICE TPC, can be as low as a few $\mathrm{fC}$. It is then delivered on the detector impedance which, to a very good approximation, is a pure capacitance of the order of a few pF. The shape of the signal tail, which is due to the motion of the positive ions, is rather complex and depends on the details of the chamber and pad geometry. This tail, causing pile-up effects, sets the main limitation to the maximum track density at which a MWPC can be operated [ALME et al., 2010].

The readout of the signal is done by 557568 pads that form the cathode pad plane of the readout chambers. The signals from the pads are passed to four 356 Front-End 


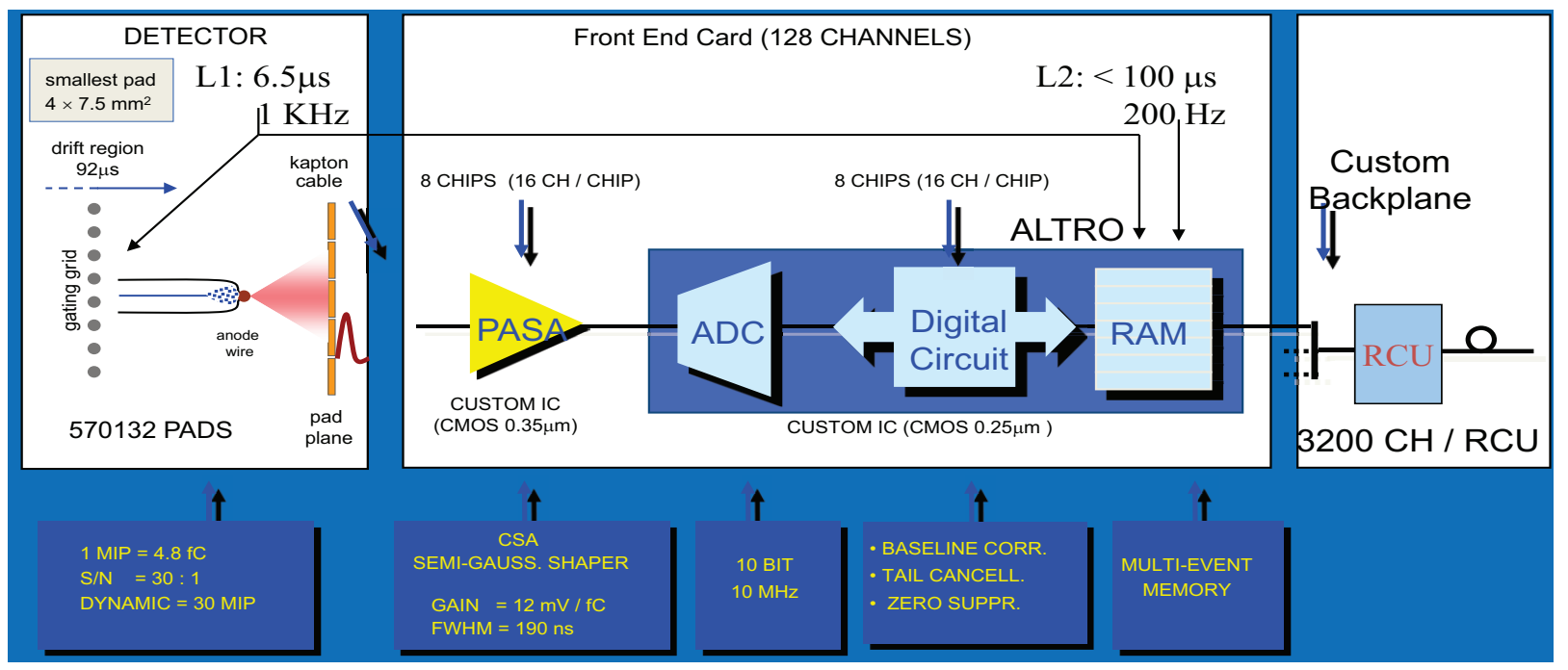

Figure 1.6: An overview of the ALICE TPC front end electronics

Cards (FECs), located 7cm away from the pad plane, via flexible Kapton cables. In the FECs a custom ASIC (Applications Specific Integrated Circuit) with 16 channels of charge sensitive amplifiers, named PASA [SOLTVEIT et al., 2012], transforms the charge signal induced in the pads into a differential semi-Gaussian voltage signal that is fed to the input of the ALTRO ASIC [MUSA, 2003]. The circuit has a conversion gain of $12 \mathrm{mV} / \mathrm{fC}$, an output dynamic range of $2 \mathrm{~V}$, and produces a differential semiGaussian pulse with a shaping time (FWHM) of 190ns. Each ALTRO contains 16 channels operating concurrently that digitize and process the input signals. The ALTRO chip contains 16 times the TSA 1001 ADC from ST Microelectronics in the form of 8 ADC IP blocks. This ADC has differential inputs and work up to a clock frequency of $10 \mathrm{MHz}$ with a resolution of 10 bits, while consuming only $12 \mathrm{~mW}$. The digitized signal is then processed by a set of circuits that perform the baseline subtraction, tail cancellation, zero-suppression, formatting and buffering into the ALTRO ASIC. The readout can take place at any time at a speed of up to $200 \mathrm{MByte} / \mathrm{s}$ through a 40 bit-wide backplane bus linking the FECs to the Readout Control Unit (RCU), which interfaces them to the Data AcQuisition (DAQ), the Trigger and the Detector Control System (DCS) [ALME et al., 2010].

\subsection{MWPC limitations}

The MWPC technology of the ALICE TPC has several operational limitations described bellow [ALICE, 2013]:

- The maximum drift time of ions from the amplification region to the gating grid of 
the TPC MWPC is about $180 \mu \mathrm{s}$. In order to provide efficient ion gating, the gating grid must be closed for $180 \mu \mathrm{s}$ after the end of the electron drift. Additionally, a $100 \mu \mathrm{s}$ past protection is applied to avoid event pile-up. This sets a first limitation of about $3.5 \mathrm{kHz}$ to the maximum readout rate of the present TPC.

- The anticipated particle rates at the wire chambers would reach $100 \mathrm{kHz} / \mathrm{cm}^{2}$, for which space charge effects in the amplification region result in a few percent gain drop, thus deteriorating the $\mathrm{dE} / \mathrm{dx}$ performance of the detector.

- Operating the TPC in ungated mode, i.e. leaving the gating grid continuously open, would result in a severe buildup of space charge in the drift volume due to back drifting ions.

To overcome the limitations of the MWPCs the Gas Electron Multiplier (GEM) was introduced by Fabio Sauli in 1997 [SAULI, 1997]. The GEM is a micro-pattern gas detector (MPGD) and consists of a thin, metal-clad polymer foil, chemically pierced by a high density of holes (typically 50 to 100 per $\mathrm{mm}^{2}$ ). On application of a difference of potential between the two electrodes, electrons released by radiation in the gas on one side of the structure drift into the holes, multiply and transfer to a collection region. Each hole acts as an individual proportional amplifier. The multiplier can be used as detector on its own, or as a preamplifier in a multiple structure; in this case, it permits to reach large overall gains in harsh radiation environment. The GEM structure diagram taken from CERN Gas Detector Development group is shown in the Figure 1.7. In the GEMs, most of the ions are collected rapidly in the upper side of the GEM foil and the signal induced in the pads is created by the movement of electrons between the foil and the pads. Therefore the induced signal has a negative polarity [KARLEN et al., 2001].

\subsection{ALICE update}

The management committee of the LHC accelerator has just approved a program of updates for the ALICE experiment [ALICE, 2013]. Among the upgrades planned for the coming years of the ALICE experiment is to improve the resolution and tracking efficiency maintaining the excellent particles identification ability, to increase the readout event rate to $50 \mathrm{KHz}$. To this end, the present MWPC based readout chambers will 


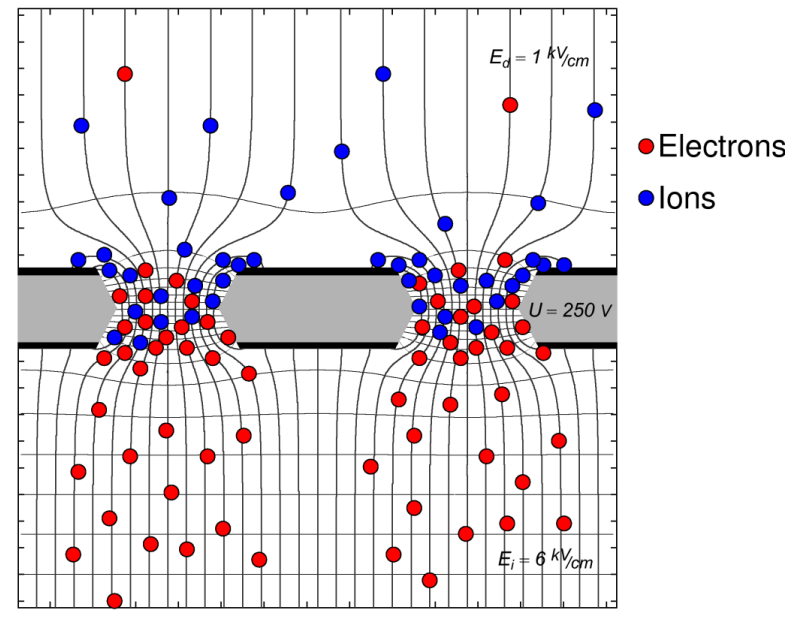

Figure 1.7: GEM structure diagram taken from CERN Gas Detector Development group [CERN..., ]

be replaced by GEM detectors which feature intrinsic ion blocking without additional gating and exhibit excellent rate capabilities. This implies also the replacement of the existing front-end electronics to accommodate the GEM new requiriments: to support inverted signal polarity and to provide the full flexibility of a continuous readout. The existing field cage as well as most of the services of the present TPC will remain. The main limitations of the PASA and ALTRO ASICs used in the read-out electronic are [ALICE, 2013]:

- PASA shaping-amplifier reads signals with a positive polarity, while its dynamic range for negative polarity, as those produced by GEM detectors, is limited to about 100 times the noise and, therefore, not adequate to accommodate the TPC signals (dynamic range 900:1).

- ALTRO does not support continuous read-out: TPC signals sampling and data storage on memory cannot happen simultaneously. Continuous read-out is a requirement for TPC upgrade.

- The read-out unit requires increasing the number of channel per chip from 16 (currently used) to 32 .

\subsection{Justificative}

In the context of the R\&D for the Linear Collider TPC, a new chip has been developed, named Super-ALTRO that overcomes the first limitation. The Super-ALTRO is a further evolution of the ALTRO chip. It combines in a single silicon die the PASA and 
the ALTRO functionality, includes more processing functions and can operate with both positive and negative polarities. The new readout system for the upgraded TPC can be built on the basis of the current TPC readout and the Super-ALTRO chip. However, a new version of the Super-ALTRO chip, that supports a continuous readout scheme, is needed. In this concern we would like to mention that the continuous readout might become a limitation for the integration of the PASA and digital chip in the same substrate [ASPELL et al., 2013].

High power consumption of the ADC analog blocks and the main amplifier of PASA are the main limitations for full integration of 32 channels. The power consumption of super ALTRO is $47 \mathrm{~mW}$ per channel. A chip with 32 super-ALTRO channels would dissipate about 1.5W. High power dissipation complicates the integration of noise sensitive analog circuits and digital circuits, because noise coupling to sensitive analog nodes is critical. The PASA chip needs $90 \mathrm{~dB}$ on the input of isolation considering $20 \mathrm{uV}$ of input signal amplitude and $1.5 \mathrm{~V}$ of voltage supply. The area consumption of the superALTRO channel is another limitation for full integration of 32 channels. The current area per channel is $4 \mathrm{~mm} 2$ and for 32 channels then the total chip area will be $128 \mathrm{~mm}^{2}$ [ASPELL et al., 2013]. For large size $\left(>100 \mathrm{~mm}^{2}\right)$ chips the yield and number of chip per wafer fall dramatically. The ALICE experiment needs about 1.5 million of channels about 57.000 chips.

To overcome these limitations has been proposed the design, fabrication and experimental test of new ASIC named SAMPA, which can be used for signals detection in the TPC. The circuits will support both positive and negative polarities, with 32 channels per chip and continuous data readout with smaller power consumption than the previous versions. The SAMPA ASIC is being designed by the VLSI design group of the Polytechnic School of the University of São Paulo.

The MCH (Muon Chambers) system is other important detector of the ALICE experiment which request a read-out electronic upgrade [COLLABORATION, 2013]. The SAMPA chip will be a common readout front-end chip for the TPC and $\mathrm{MCH}$. It will reduce the implementation cost of the electronic upgrade project. Because TPC and $\mathrm{MCH}$ have different specifications of detector capacitance, noise, sensitivity and peaking time, the readout front-end should be configurable for both detectors. 
The CMOS technology offers more advantages than other technologies as Bipolar or GaAS (Gallium Arsenide) to implement read-out frontend ASICs, among which are highlighted: integration of analog and digital circuits in the same die and Lower power dissipation with high integration levels. Furthermore, CMOS devices enables to package more functions using the same manufacturing technology in a single die, thus reducing the manufacturing cost and working at faster speed.

This work aims to design, fabrication and experimental test of an read front-end in CMOS technology which will be used in the new SAMPA ASIC. The proposed frontend is composed of: Charge Sensitive Amplifier, Semi-Gaussian shaper and Analogto-digital converter. The designed circuit must achieve the specifications requested by the Time Projection Chamber and the Muon Tracking Chamber of the ALICE experiment with. In order to obtain an ASIC integrating 32 channels per chip, a low area low power consumption front-end with configurable polarity (positive/negative), input dynamic range, peaking time and sensitivity so the new ASIC can be used in both chambers.

\subsection{Objectives}

1. Design, fabricate and test a Charge Sensitive Amplifier achieving the specification requested by the TPC and MCH of the ALICE experiment with configurable polarity (positive/negative) and input dynamic range in 130nm CMOS technology.

2. Develop new techniques for noise and PSRR improvement of the proposed frontend.

3. Design, fabricate and test a Semi-Gaussian shaper fulfilling the specification requested by the TPC and $\mathrm{MCH}$ of the ALICE experiment with configurable peaking time and sensitivity in 130nm CMOS technology.

4. Integrate and fabricate the complete front-end composed of Charge Sensitive Amplifier and Semi-Gaussian shaper.

5. Perform the experimental characterization of each function block that constitute the front-end.

6. Perform the experimental characterization of the designed front-end. 


\subsection{Contributions of this thesis}

The work developed in this thesis introduces the following contributions:

- A new analysis and equations of the noise contribution of each transistor of CSA traditional cascode topology are presented in Chapter 3 . In several works only the noise components generated by the CSA input transistor (flicker and channel thermal noise) are studied, but the noise components from others transistors of the CSA are disconsidered [GASPARI, 2012][SOLTVEIT et al., 2012][PROSPECTS. .., 2002]. The presented analysis shows which are the more important noise components and which are the components with low impact on the total noise. This study shown that the noise from the bias current mirror has a significant contribution on the total noise, so it can not be ignored. Based on the introduced equations, the design considerations for minimizing the total noise specification are discussed.

- Two new techniques for the bias noise cancellation are presented in Chapter 4 which are based on the traditional CSA cascode topology. The small signal model of the proposed topologies are studied, and the equation that describes the conditions for the cancellation effect are given. The theoretical equations are validated by simulation results of the designed CSA and experimental test of 3 samples of a produced chip.

- A new analysis of the CSA PSRR degradation and its dependency with the detector capacitance of the traditional CSA topology is presented in Chapter 4. In previous works the PSRR is measured for a specific values of detector capacitance[GASPARI, 2012], but PSRR degradation in term of the detector capacitance is ignored. This work illustrates the PSRR degradation with a theoretical analysis and equation which were validated by electrical simulations.

- The analysis of the PSRR improvement of the CSA and of the front-end with the proposed topologies for bias noise cancellation is discussed in chapter 4 . This study demonstrates that the proposed topologies besides noise canceling they also allow to increase the CSA PSRR and as a consequence the front-end PSRR. 


\subsection{Thesis summary}

This thesis is organized in 6 chapters, where the introduction was presented in this chapter. The theoretical aspects of the readout front-end for gaseous detectors are presented in chapter 2, and the specifications of pulse timing, noise, crosstalk, sensitivity, input dynamic range and non-linearity are also illustrated. Furthermore, the challenges and requirements for the proposed front-end design from the system point of view are discussed in chapter 2 .

Chapter 3 presents the selected front-end architecture and the considerations and requirements for the circuit design. It lists the theoretical equations to model the electrical characteristics of the CSA circuit as cross-talk, noise, open-loop gain, residual gain, input impedance, frequency response and rise time. A noise analysis including all CSA circuit transistors, not only the noise generated by the input CSA transistor, are also discussed. The flow used for the CSA and Semi-gaussian shaper design and some simulations results are presented from the second half of chapter 3.

The proposed techniques for the bias noise cancellation are presented in Chapter 4. The small signal model of the proposed topologies are studied, and the equations that describe them electrical characteristics are given. The analysis of the PSRR improvement of the CSA and of the front-end with the proposed topologies for bias noise cancellation are also discussed in chapter 4 .

Chapter 5 shows the experimental results obtained from a produced chip with 5 channel of the designed front-end, which are compared with the simulations. Finally, the conclusion and future works are presented in chapter 6 . 


\section{Chapter 2}

\section{Readout front-end for gaseous}

\section{detectors}

In the introduction section, the motivation and justification of the design, fabrication and experimental test of the new SAMPA ASIC to be used in the TPC and $\mathrm{MCH}$ readout electronic of the ALICE experiment has been presented. In this section, the block diagram of a read-out front-end are presented as well as its electrical specifications and output signal characteristics. The charge signal generated on the pad plane can be modeled as the parallel of a short pulse current source (typically 1 ns of duration) and a capacitance for detector capacitance modeling[SPIELER, 2005]. Read-out electronic for fast low amplitude pulse signal processing is very complex, and an analog pre-processing should be done before the analog-to-digital conversion and the digital processing. An analog read-out front-end is connected to each detector pad directly. It is composed of a Charge sensitive Amplifier (CSA), a Semi-Gaussian pulse shaper and an analog-to-digital converter. A typical block diagram of the electronic read-out

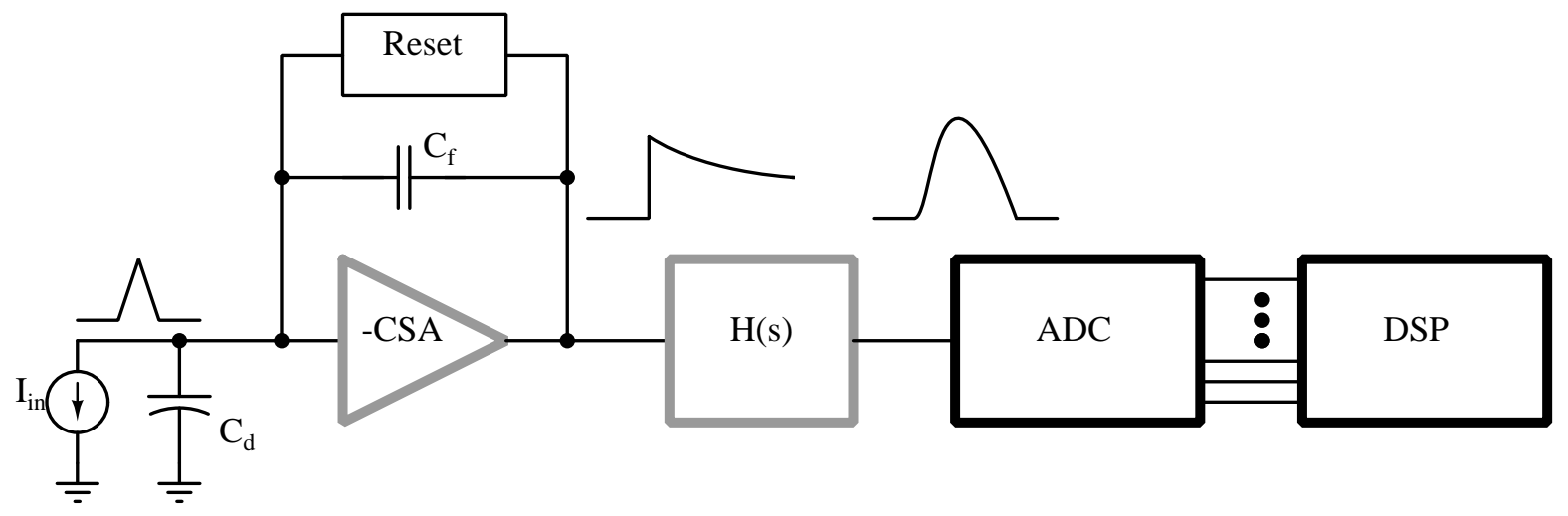

Figure 2.1: Typical block diagram of the electronic read-out system of a gaseous detectors. 
system of a gaseous detectors is shown in Figure 2.1. The generated fast charge signal $(Q)$ on the pad plate is integrated on a feedback capacitance $C_{f}$ of the CSA which produces a step voltage signal at the output of the CSA with amplitude equal to $Q / C_{f}$. The step signal is fed to a band-pass filter $\mathrm{H}(\mathrm{s})$ constituted by a first order high-pass filter (differentiator) and a $n$ order low pass filter (integrator). $\mathrm{H}(\mathrm{s})$ generates a SemiGaussian pulse with amplitude proportional to the input charge in response to the step input signal produced on the CSA output. A reset strategy to avoid saturation of the input signal integration is used on the CSA. There are 3 options to do the reset function [SPIELER, 2005][SPIELER, 2003]: (1) a discharge resistor, (2) a switch activated after a event, (3) a current source to discharge the feedback capacitor continuously. The output voltage in frequency domain of the Semi-Gaussian shaper is given by[SANSEN; CHANG, 1990b]:

$$
V_{\text {out }}(s)=\left[\frac{s \tau_{0}}{1+s \tau_{0}}\right]\left[\frac{A}{1+s \tau_{0}}\right]^{n} \cdot \frac{Q}{s C_{f}}
$$

where $\tau_{0}$ is the time constant of the differentiator and integrator, and $A$ is the dc gain of the integrator. The number of integrators $(n)$ is called the order of the semi-Gaussian shaper. In the frequency domain, the output signal of the pulse shaper is given by the product of the transfer function $\mathrm{H}(\mathrm{s})$ and the Laplace transform of the step signal that is equal to $Q / s C_{f}$. By taking the inverse Laplace transform of the product, the output signal in the time domain is obtained[SANSEN; CHANG, 1990b]:

$$
V_{\text {out }}=\frac{Q A^{n} n^{n}}{C_{f} n !}\left(\frac{t}{\tau_{s}}\right)^{n} e^{-n t / \tau_{s}}
$$

where $\tau_{S}$, called peaking time, is defined by $\tau_{S}=n \tau_{0}$. It can be verified that the peak amplitude is reached at the peaking time $\tau_{s}$. The peak amplitude of $V_{\text {out }}$ is given by:

$$
V_{\text {out }}^{\max }=\frac{Q A^{n} n^{n}}{C_{f} n ! e^{n}}
$$

A semi-Gaussian pulse waveforms with normalized amplitude and peaking time in 160 ns and $n=2,4,6$ are shown in Figure 2.2a. This figure shows the reduction of the return to baseline time when the low pass filter order is increased. Figure 2.2b illustrates the pulse waveform variation for $40 \mathrm{~ns}, 80 \mathrm{~ns}$ and $120 \mathrm{~ns}$ of peaking time $(n=6)$ with normalized amplitude. The pulse waveforms presented in Figure 2.2 were gener- 


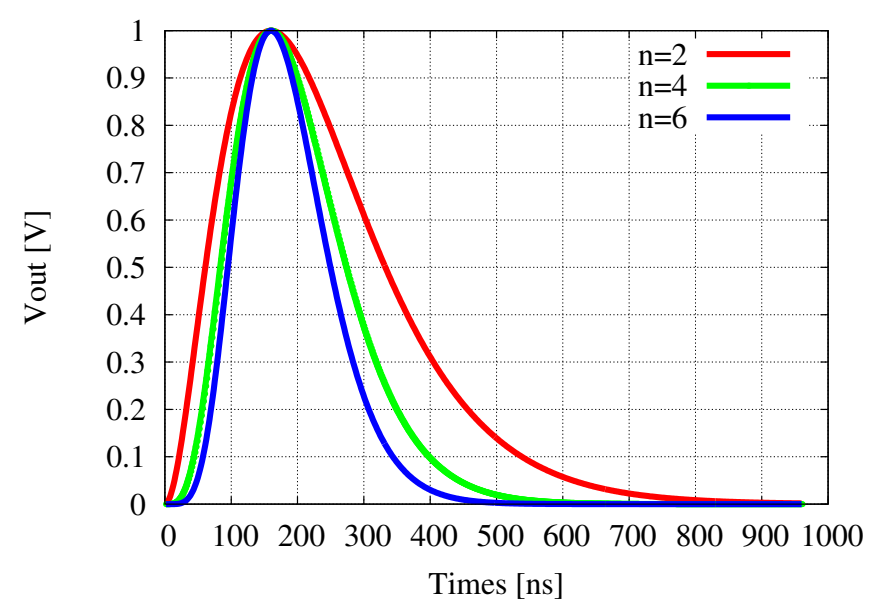

(a)

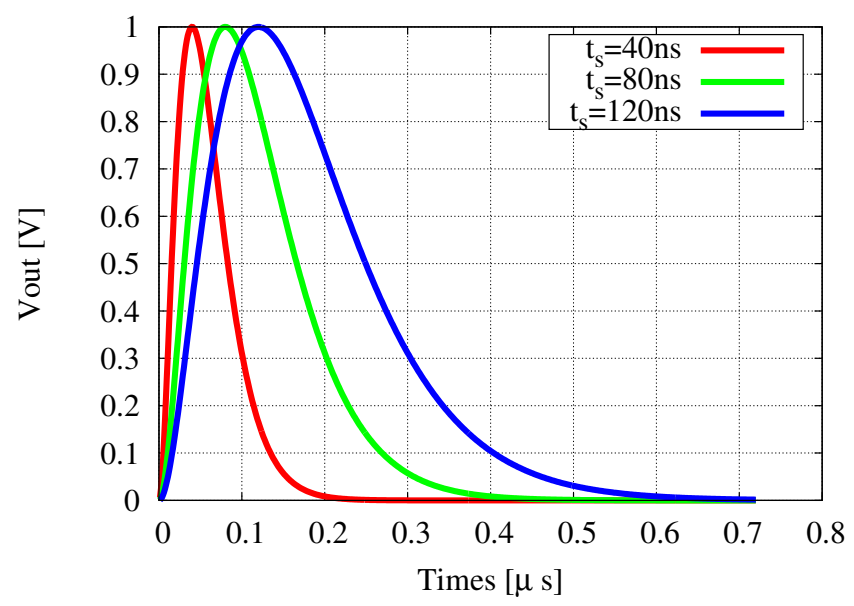

(b)

Figure 2.2: (a) A semi-Gaussian pulse waveforms with normalized amplitude for 160ns of peaking time and $n=2,4,6$; (b) pulse waveform variation for $40 n s$, $80 n$ s and 120 ns of peaking time $(n=6)$ with normalized amplitude.

ated from the equation (2.2). The main electrical specifications of an analog front-end for gaseous detectors readout are defined below.

\subsection{Readout front-end specifications}

\subsubsection{Sensitivity}

The front-end sensitivity is the amplitude of the output pulse (Volts [V]) generated by a determined input charge (Coulomb [C]) and it is given by:

$$
\text { Sensitivity }=\frac{\text { Pulse Amplitude }}{\text { Input Charge }}\left[\frac{V}{C}\right]
$$

Generally, the sensitivity specification is defined in $[\mathrm{mV} / \mathrm{fC}]$. 


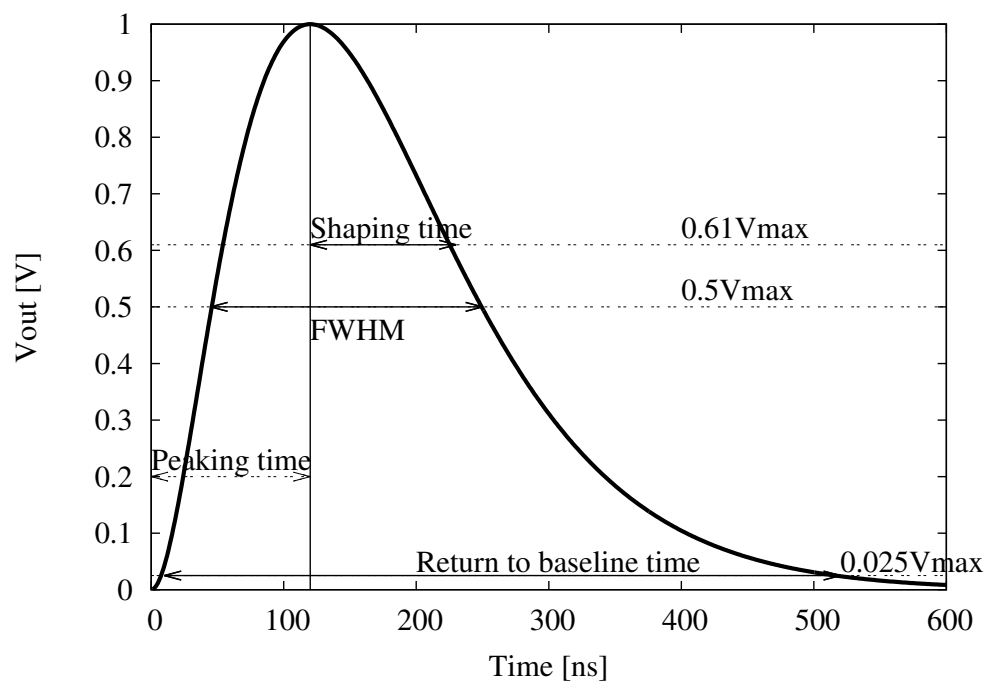

Figure 2.3: FWHN, shaping time and peaking time specifications of a semi-Gaussian pulse.

\subsubsection{Pulse time specifications}

The full width at half maximum (FWHM) value of the semi-Gaussian pulse time distribution is often used as a measure of the overall timing uncertainty in the measurement system and is called the time resolution. The time required for the shaped pulse to reach its maximum amplitude is often called the peaking time. Shaping time can be defined at $61 \%$ of the peak value. FWHN, shaping time and peaking time of a semiGaussian pulse are indicated in Figure 2.3[INIEWSKI, 2010][BLUM; ROLANDI, 2008]. The return-to-baseline time is defined as the difference between the times at which the absolute value of the linear channel output first crosses $2.5 \%$ of its maximum and when it again crosses $2.5 \%$ of maximum as it decays to baseline[LOINAZ; WOOLEY, 1995].

\subsubsection{Crosstalk}

Signal coupling between adjacent channels due mainly to parasitic capacitances is presented in analog front-ends for gaseous detectors. If a determined charged is applied on channel 2 of a 5 adjacent front-end channels, semi-Gaussian pulses with different energies will be detected on each channel output. This effect is illustrated in Figure 3.9 for a 5 front-end channels array. The crosstalk specifications between channel 2 and 3 is described by the equation bellow:

$$
\text { CrossTalk }_{2-3}=\frac{A_{3}}{A_{2}} \times 100[\%]
$$

where $A_{2}$ and $A_{3}$ are the output pulse amplitude peak of the channel 2 and 3 respec- 


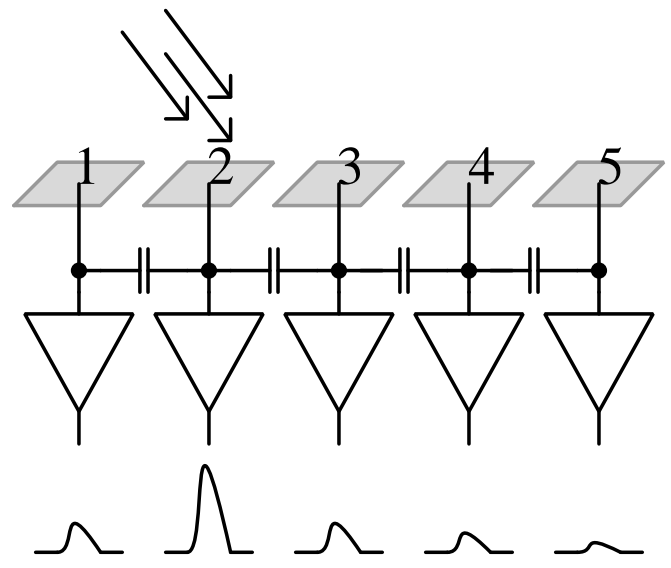

Figure 2.4: Pulse coupling between adjacent channels for a 5 channels front-end array. tively. The cross-talk is measured in \% or $\mathrm{dB}$.

\subsubsection{Equivalent Noise Charge}

The noise performance of a detector readout system is expressed by the Equivalent Noise Charge (ENC). The ENC is defined as the ratio of the total integrated RMS noise $\left(V_{R M S}\right)$ at the pulse shaper output to the signal amplitude due to a one electron charge $q$. The output amplitude due to one electronic charge is given by $V_{\text {out }} / \mathrm{Q}$ where $\mathrm{Q}$ is a determined input charge and $V_{\text {out }}$ is the output pulse amplitude. The ENC in electrons unit $[e]$ can be described by [CHONG; SANSEN, 1991]

$$
E N C=\frac{V_{R M S} \cdot Q}{q \cdot V_{\text {out }}}[\mathrm{e}]
$$

As definition implies, the ENC depends on the characteristics of both the CSA and of the pulse shaper.

\subsubsection{Input dynamic range}

The signal-conditioning chain, or generally any amplifier, can only be used within a defined signal-amplitude range [INIEWSKI, 2010]. The lower limit is the noise level, and signals below the noise level are undetectable. The upper hard limit is the maximum output voltage. As the output signal of the amplifier increases, the output stages start to compress the signal before reaching the maximum output voltage level. The effect is a decreased gain for higher signal amplitudes, causing signal distortion and, in the worst case, signal clipping and linearity errors. The input dynamic range of a detector readout front-end describes the input charge range supported without signal saturation (non- 


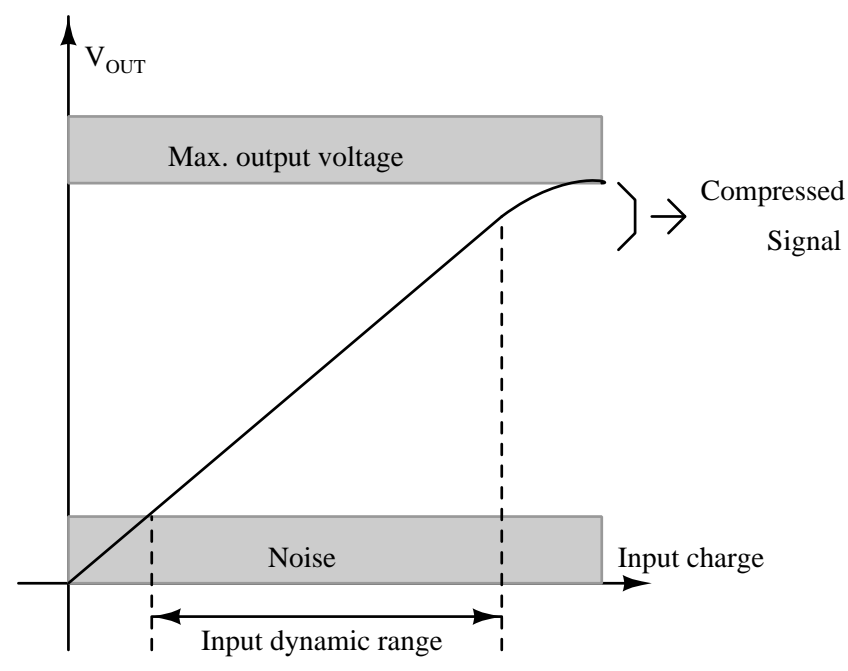

(a)

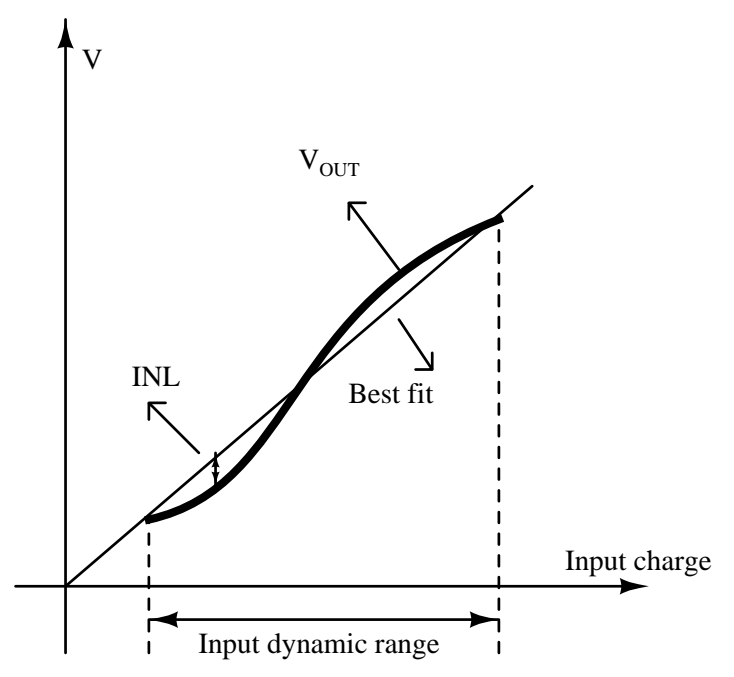

(b)

Figure 2.5: (a) Input dynamic range definition; (b) Integrated non-Linearity (INL) specification. linear circuits operation) or signal limitation due to the noise floor. The input dynamic range definition is illustrated in Figure 2.5a.

\subsubsection{Non-Linearity}

The transfer function of the front-end output pulse amplitude $\left(V_{\text {out }}\right)$ vs input charge (Q) is ideally linear. The non-linear effects of the transistor based circuits and signal saturation are estimated by the Integrated non-Linearity (INL) specification. The INL is the ratio of the maximum deviation of the real output function from an ideal straight line (best fit straight line for the taken samples) to the input dynamic range (Figure 2.5b). The INL is expressed by:

$$
I N L=\frac{\text { Maximum Deviation }}{\text { Input dynamic range }} \times 100[\%]
$$

\subsubsection{Pole-zero cancellation}

The Semi-Gaussian output pulse is obtained under the assumption that the output signal of the CSA is an ideal voltage step. However, the output step signal return to its original level very slowly with the long time constant of the CSA. This slow decay of the step signal will results in a negative overshoot or an undershoot in the Semi-Gaussian pulse due to the differentiation function of the shaper. The undershoot phenomenon will strongly limit the counting rate behavior[CHONG; SANSEN, 1991]. At medium to 


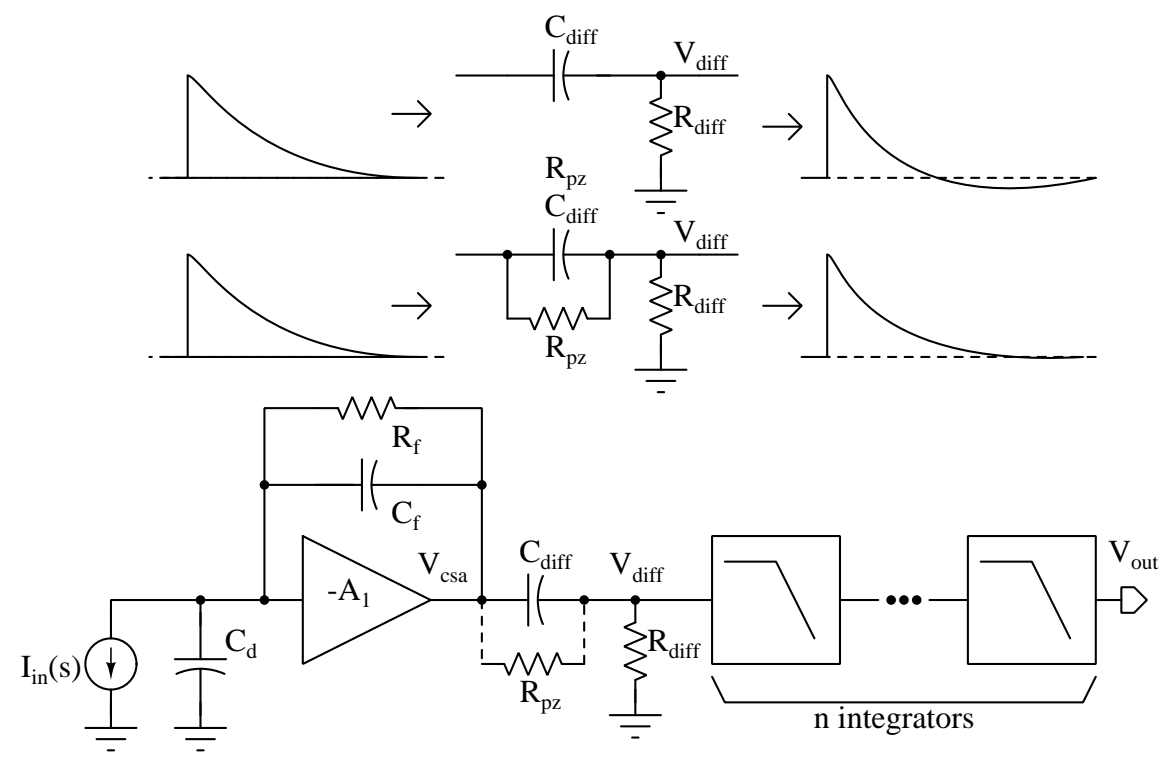

Figure 2.6: Diagram of the pole-zero cancellation circuit.

high counting rates, a substantial fraction of the amplifier output pulses will ride on the undershoot from a previous pulse. The apparent pulse amplitudes measured for these pulses will be significantly lower, which deteriorates the energy resolution.

Most shaping amplifiers incorporate a pole-zero cancellation circuit to eliminate this undershoot. The benefit of pole-zero cancellation is improved peak shapes and resolution in the energy spectrum at high counting rates. Figure 2.6 illustrates the pole-zero cancellation circuit, and its effect. The CSA output signal is applied to the input of the $\mathrm{CR}$ differentiator circuit. The output pulse from the differentiator exhibits the undesirable undershoot. To cancel the undershoot, a resistor $R_{p z}$ is added in parallel with the capacitor $C_{d i f f}$ with the requirement $t_{p z}=C_{f} R_{f}=C_{d i f f} R_{p z}$. The result is an output pulse exhibiting a simple exponential decay to baseline with the desired differentiator time constant. Exact pole-zero adjustment is critical for good energy resolution. The output voltage of the CSA is now modified to be:

$$
V_{\text {out }}(s)=\frac{\left(s+1 / \tau_{p z}\right)}{\left(s+1 / \tau_{o}\right)}\left[\frac{A}{1+s \tau_{0}}\right]^{n} \frac{Q}{C_{f}\left(s+1 / \tau_{1}\right)}
$$

where the last product factor represents the exponential decaying step signal of the CSA with decay time constant $\tau_{1}=R_{f} C_{f}$. By choosing the time constant $\tau_{p z}=\tau_{1}$, the above equation reduces to the equation (2.1) 


\subsection{Read-out front-end of the ALICE TPC}

As mentioned in the introduction chapter, the ALICE TPC readout of the signal is done by pads that form the cathode pad plane of the readout chambers via the Front-End Cards (FECs). In the FECs the PASA ASIC transforms the charge signal induced in the pads into a differential semi-Gaussian voltage signal that is fed to the input of the ALTRO ASIC. The digitized signal is then processed and the readout can take place at any time at a speed of up to $200 \mathrm{MByte} / \mathrm{s}$ through a 40 -bit-wide backplane bus linking the FECs to the Readout Control Unit (RCU). The architecture and specifications of the ALTRO and PASA ASIC are presented below.

\subsubsection{PASA ASIC}

The PASA ASIC integrates 16 identical Charge Sensitive Amplifiers (CSAs) followed by a pole-zero cancellation network and a shaping amplifier. A simplified block diagram of the signal processing chain is shown in Figure 2.7. The positive polarity CSA, with a capacitive and resistive feedback connected in parallel, is followed by a pole-zero cancellation network with a self-adaptive bias network, a CR-high-pass filter, two $(\mathrm{RC})^{2}$-bridged-T low pass filter filters, a common-mode feed-back network and two quasi-differential gain-2 amplifiers. The circuit is optimized for a detector capacitance of 18-25pF [SOLTVEIT et al., 2012]. The amplifier topology is based on a single-ended folded cascode amplifier followed by a source follower. As seen in Figure 2.7, an NMOS transistor, which is operated in the subthreshold region to implement a large resistor, is connected in parallel to the feedback capacitor $C_{f}$. The purpose of this resistor is to avoid saturation of the CSA by continuously discharging the feedback capacitance. Still, the relatively long discharge time constant of the CSA may cause signal pile-up. For this reason, the CSA is followed by a pole-zero cancellation network $\left(\mathrm{M}_{p z}\right.$ and $\left.C_{d i f f}\right)$, which is combined with the $C_{d i f f} R_{d i f f}$-filter stage. The signal is then amplified and further shaped by two second order bridged-T filters to optimize the signal-to-noise ratio and to limit the signal bandwidth. In the last PASA stage the signal levels are adjusted to match the input of the ALTRO chip. The chip was manufactured in the C35B3C1 $0.35 \mu \mathrm{m}$ CMOS technology featured by Austriamicrosystems. It has a total an area of $18 \mathrm{~mm}^{2}$ 


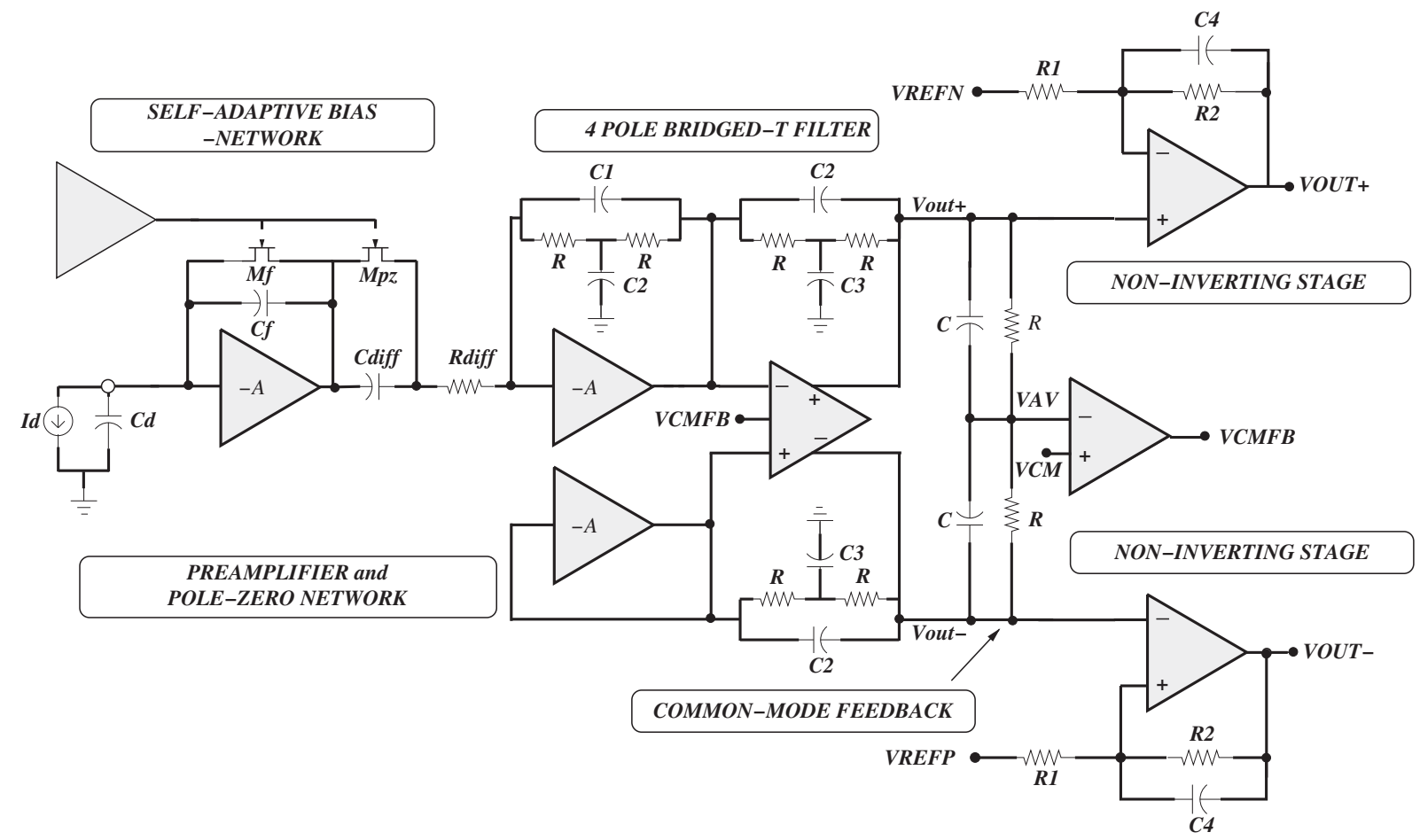

Figure 2.7: Simplified block diagram of the signal processing chain of the PASA channel. Figure taken from [SOLTVEIT et al., 2012]

\subsubsection{ALTRO ASIC}

ALTRO is a mixed-signal custom integrated circuit containing 16 channels operating concurrently and continuously on the analog signals coming from 16 independent inputs. It is designed to process a train of pulses sitting on a common baseline. When a Level-1 (L1) trigger is received, a predefined number of samples is processed and temporarily stored in a data memory (acquisition). This acquisition is frozen if a Level-2 (L2) trigger is received; otherwise, it is overwritten by t e next Level-1 arrives. There is the possibility of having pre-trigger samples or to delay the acquisition by a programmable number of cycles[BOSCH et al., 2002]. As shown in Figure 2.8, the ALTRO chip contains 16 acquisition channels each comprised of three main units: an ADC, a Digital Processor and a Data Memory. A short description of these three blocks is given hereafter. The ADC is the 10-bit 25-MSPS TSA1001 from ST Microelectronics. The TSA 1001 offers $25 \mathrm{~mW}$ of power consumption at 25-MSPS and $2.5 \mathrm{~V}$ supply. A specific power-adaptive feature allows reducing this figure, when the ADC is operating at lower sampling rates, by means of an external polarisation current. The ADC has differential inputs with $1 \mathrm{Vpp}$ range and it is based on a pipelined architecture with 1.5bit/stage[BOSCH et al., 2002]. After digitalization, a Baseline Correction Unit is able 


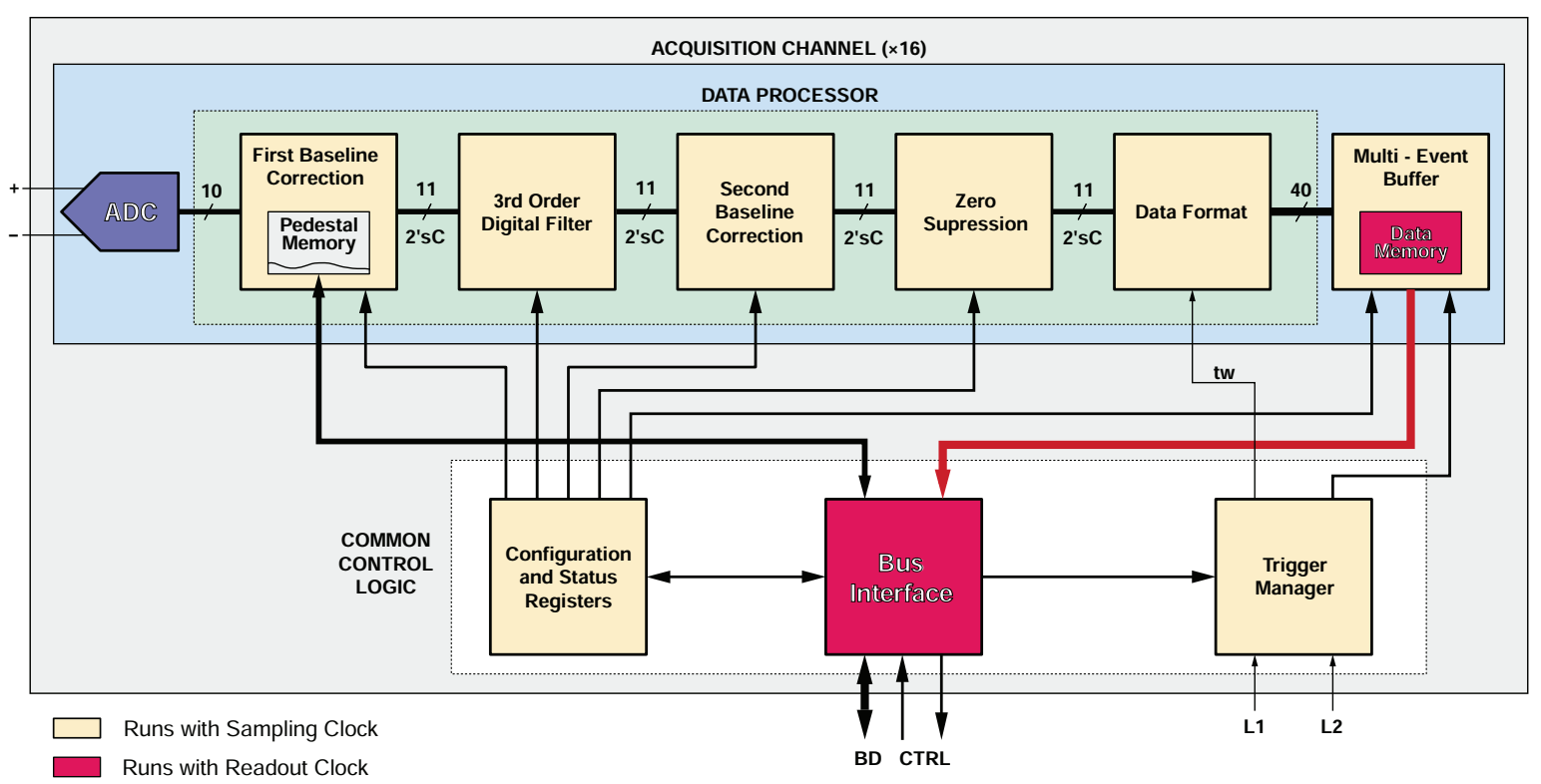

Figure 2.8: Block diagram of the ALTRO chip.

to perform channel-to-channel gain equalization and to correct for signal non-linearity and baseline drift due to temperature variations. It is also able to adjust DC levels and to remove systematic spurious signals by subtracting a pattern stored in a dedicated memory (Pedestal Memory). The next processing block is a 18-bit, fixed point, 3rd order IIR Digital Filter acting in the time-domain and whose function is described in [8]. A second correction of the baseline, based on a moving average filter removes nonsystematic perturbations of the baseline that are superimposed to the signal. Eventually, a Zero Suppression module removes all data that is below a certain threshold, except for a specified number of pre-samples and post-samples around each pulse. When the pulses are closer than a given distance, they are grouped in the same data packet. This produces a certain number of non-zero data packets, thus, reducing the overall data volume. Each data packet is formatted with its time stamp and size information, so that the original data can be reconstructed afterwards. The output of the Data Processor is stored in the Data Memory, which has a capacity of 5-Kbyte and is able to store 8 full acquisitions. The data readout is performed at $60 \mathrm{MHz}$ through a 40-bit wide bus, yielding a total bandwidth of 300-Mbyte/s. The ALTRO chip has two clock domains: 1) Sampling and Data Processing domain; 2) Data Memory readout and bus interface domain. The ALTRO chip is implemented in the ST $0.25 \mu \mathrm{m} \mathrm{HCMOS-}$ 7 process, has an area of $64 \mathrm{~mm}^{2}$ and a power consumption of $320 \mathrm{~mW}$ when the 16 channels are acquiring at a $10 \mathrm{MHz}$ rate. The measurements show a resolution better 


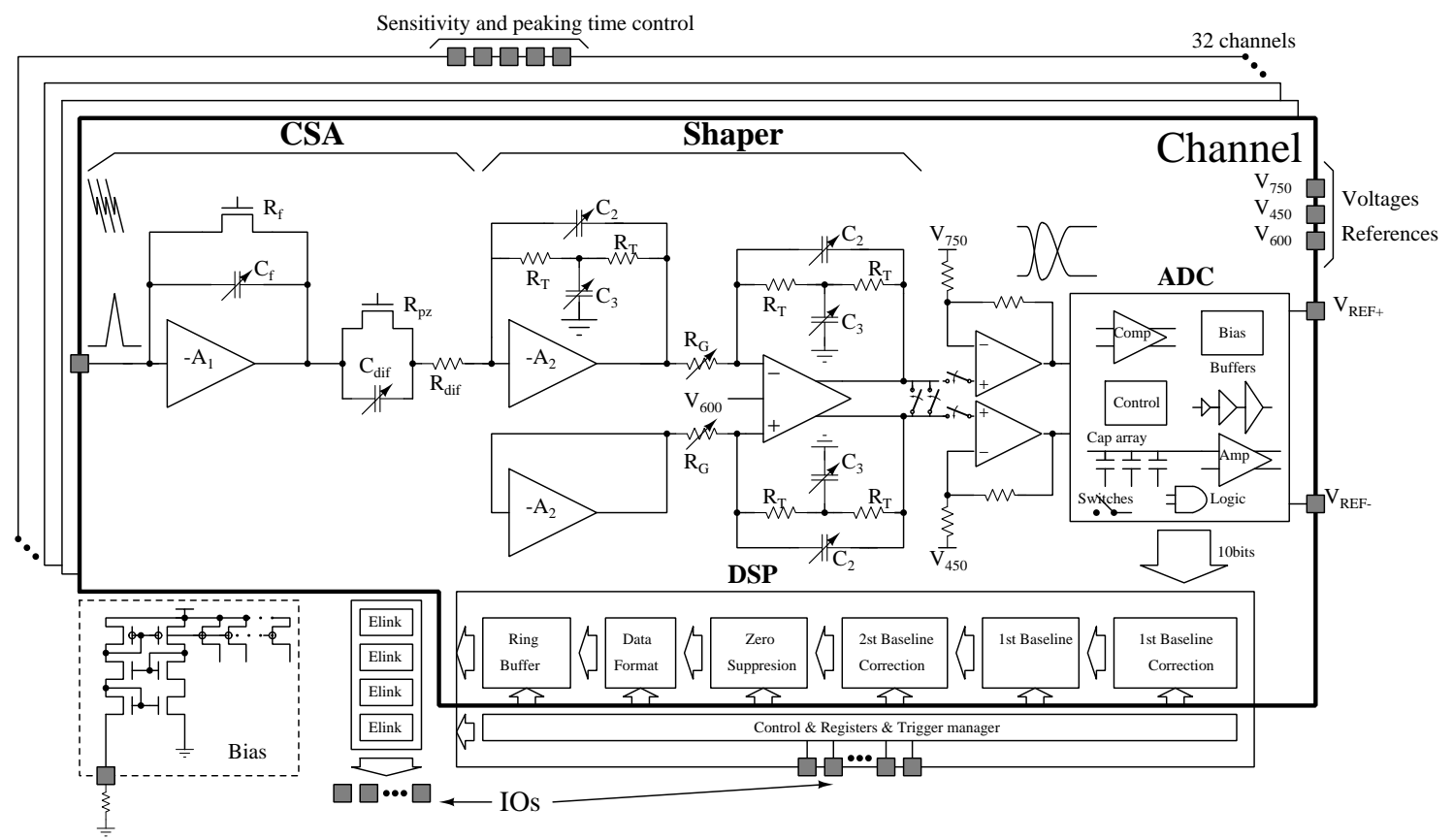

Figure 2.9: Block diagram of the SAMPA ASIC.

than 9.5 ENOB[BOSCH et al., 2002].

\subsection{New front-end ASIC: SAMPA}

In SAMPA contains positive/negative polarity Charge Sensitive Amplifiers (CSA), which transform the charge signal into a differential semi-Gaussian voltage signal, that is digitized by a 10-bit 10 Msamples/s ADC. After the ADC a digital signal processor eliminates signal perturbations, distortion of the pulse shape, offset and signal variation due to temperature variations. SAMPA contains 32 channels per chip that concurrently digitizes and processes the input signals as shown in Figure 2.9. The data read-out takes place continuously at a speed of up to $1.28 \mathrm{Gbps}$ by four $320 \mathrm{Mb} / \mathrm{s}$ serializations links (e-links). The data read-out can be performed in continuous mode or triggered mode. In continuous mode the read-out of a programmable number of samples is performed trigger-less if the input signal exceeds the programmable threshold value. For the TPC application a design rate of $50 \mathrm{kHz}$ with $30 \%$ occupancy is assumed. For the $\mathrm{MCH}$ the design rate is $100 \mathrm{kHz}$ and $10 \%$ occupancy. Software triggers are accepted in continuous mode for calibration and synchronization purposes. In triggered mode data read-out of programmable number of samples is performed only upon reception of an external trigger with a maximum latency $<9.6 \mu \mathrm{s}$. Triggers arriving during an active read-out will be accepted. In that case the active read-out will be extended by the new 
arriving trigger for the programmable number of samples and status information is sent to acknowledge the read-out extension. Optionally a programmable number of pre/post samples before/after the input signal crossed the threshold in continuous mode or the external trigger mode arrived can be read-out2.9.

\subsubsection{SAMPA building blocks}

The SAMPA ASIC is composed by a positive/negative polarity Charge Sensitive Amplifier (CSA) with a capacitive feedback $C_{f}$ and a resistive feedback $R_{f}$ connected in parallel, a Pole-Zero Cancellation (PZC) network, a high pass filter, two bridged-T second order low pass filters, a non-inverting stage, a 10 Msamples/s 10-bit SAR ADC and a Digital Signal Processor(DSP) block, as shown in Figure 2.9. The first shaper is a scaled-down version of the CSA and generates the first two poles and one zero. A copy of the first shaper connected in unity gain configuration is implemented in order to provide a differential mode input to the next stage. The second stage of the shaper is a fully differential second order bridged-T filter and it includes a Common-Mode Feed-Back network (CMFB). The non-inverting stage adapts the DC voltage level of the shaper output to use the full dynamic range of the ADC. It consists of a parallel connection of two equally designed Miller compensated amplifiers. The ADC is a differential 10-bit 10 Msamples/s SAR (successive approximation) ADC implemented with a low power switching technique. The DSP part is composed by digital filters, a data format unit, a ring buffer, a trigger manager block, a configuration register bank, a control state machine, and four $320 \mathrm{Mb} / \mathrm{s}$ e-links. The chip will be fabricated in 0.13 $\mu \mathrm{m}$ CMOS technology. The design specifications of the SAMPA ASIC are summarized in Table 2.1. 
Table 2.1: Specifications of the new front-end ASIC (SAMPA).

\begin{tabular}{|c|c|c|}
\hline Specification & TPC & MCH \\
\hline Voltage supply & $1.25 \mathrm{~V}$ & $1.25 \mathrm{~V}$ \\
\hline Polarity & Positive/Negative & Positive/Negative \\
\hline Detector capacitance (Cd) & $18.5 p F$ & 40pF - 80pF \\
\hline Peaking time (ts) & $80 \mathrm{~ns}$ or $160 \mathrm{~ns}$ & 300ns \\
\hline Shaping order & 4th & 4th \\
\hline Equivalent Noise Charge (ENC) & $\begin{array}{c}<600 \mathrm{e} @ \mathrm{ts}=80 \mathrm{~ns}^{*} \\
\text { or }<600 \mathrm{e} @ \mathrm{ts}=160 \mathrm{~ns}^{*}\end{array}$ & $\begin{array}{l}<950 \text { e @ Cd=40p* } \\
<1600 \text { e @ Cd=80p* }\end{array}$ \\
\hline Maximum input charge & $100 \mathrm{fC}$ or $67 \mathrm{fC}$ & $500 \mathrm{fC}$ \\
\hline Sensitivity & $20 \mathrm{mV} / \mathrm{fC}$ or $30 \mathrm{mV} / \mathrm{fC}$ & $4 \mathrm{mV} / \mathrm{fC}$ \\
\hline Non-Linearity (CSA + Shaper) & $<1 \%$ & $<1 \%$ \\
\hline Crosstalk & $\begin{array}{c}<0.3 \% @ \mathrm{ts}=80 \mathrm{~ns} \\
\text { or }<0.2 \% @ \mathrm{ts}=160 \mathrm{~ns}\end{array}$ & $<0.2 \% @ \mathrm{ts}=300 \mathrm{~ns}$ \\
\hline ADC effective input range & 2Vpp & 2Vpp \\
\hline ADC resolution & 10-bit & 10-bit \\
\hline Sampling Frequency & 10Msamples/s & 10Msamples/s \\
\hline INL (ADC) & $<0.65$ LSB & $<0.65$ LSB \\
\hline DNL (ADC) & $<0.6$ LSB & $<0.6$ LSB \\
\hline ENOB $(\mathrm{ADC})^{\star \star *}$ & $>9.2$-bit & $>9.2$-bit \\
\hline $\begin{array}{l}\text { Power consumption (per channel) } \\
\text { CSA + Shaper + ADC }\end{array}$ & $<15 \mathrm{~mW}$ & $<15 \mathrm{~mW}$ \\
\hline Channels per chip & 32 & 32 \\
\hline
\end{tabular}

${ }^{*} \mathrm{R}_{e s d}=70 \Omega$

**@ 0.5MHz, 10Msamples/s 


\section{Chapter 3}

\section{Charge Sensitive Amplifier}

In this section the requirements and consideration for the CSA design are discussed and the equations used to estimate the circuit specifications are presented.

\subsection{CSA architecture}

The amplifier topology and the input transistor (PMOS or NMOS) are critical choices for the design of a low-power low-noise CSA, since they typically represent the largest contribution to the total system noise and to power consumption. The selected topology shown in Figure 3.1 is based on a single-ended folded cascode amplifier. This configuration has been widely used for the core amplifier, since it can be easily frequency compensated and the dominant pole is determinate by the cutoff frequency of transistors in the level shifter stage and as a result a large GBW can be obtained [CHONG; SANSEN, 1991]. A NMOS voltage follower as buffer reduce the output resistance to avoid that the pole generated by the load capacitance and the output resistance does not limit the rise/fall time of the impulsive response of the CSA. A NMOS transistor input transistor was chosen because it offers more advantages than a PMOS transistor. The GBW of the amplifier must be high enough to achieve an output rise time of the order of $n s$ as response to a impulsive charge. It requires large input transistor transconductance $g_{m 1}$. NMOS transistor offers higher $g_{m 1}$ than PMOS for the same drain current and W/L proportion. NMOS transistors ensure lower values of channel thermal noise than PMOS, but has higher flicker noise [JOHNS; MARTIN, 2008]. Channel thermal noise is the major contributor to the overall system noise [CHONG; SANSEN, 1991]. If $\mathrm{SiO}_{2}$ is used for the gate oxide, PMOS transistors will have about one order of magni- 


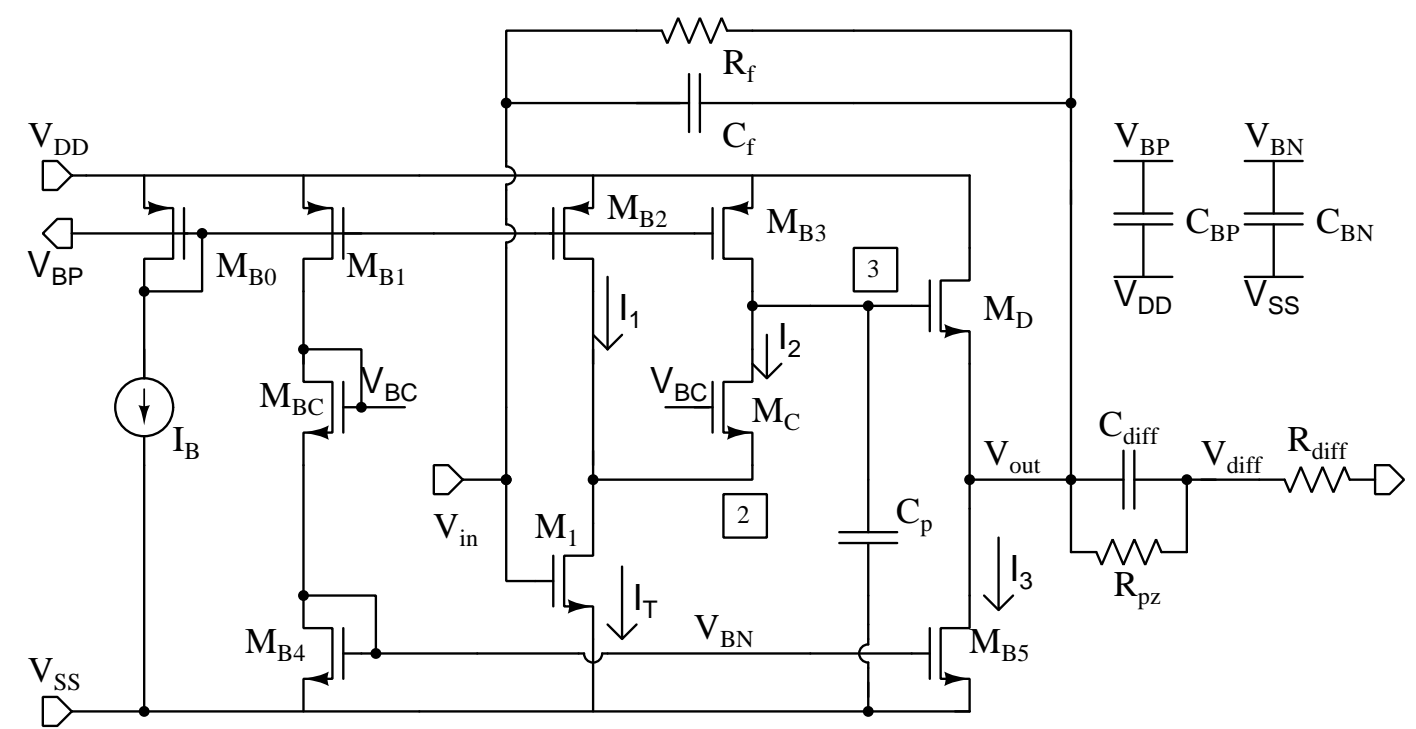

Figure 3.1: Selected topology for the amplifier of the CSA based on a single-ended folded cascode.

tude smaller gate leakage than NMOS transistors at ultra-think $t_{O x}$ [LEE et al., 2004]. The frequency compensation of the amplifier is performed by the $C_{p}$ capacitor. The capacitances $C_{B P}$ and $C_{B B}$ on the gate of the current mirror transistor were included to decouple the $A C$ components on the bias voltage.

\subsection{Design requirements of the CSA}

\subsubsection{Open loop gain}

A simple model of the CSA composed of a inverting amplifier with open loop gain $A$, a feedback integration capacitance $\left(C_{f}\right)$ and a detector capacitance $\left(C_{d}\right)$ is illustrated in Figure 3.2a. The feedback discharge resistance is neglected in this mode. The voltage

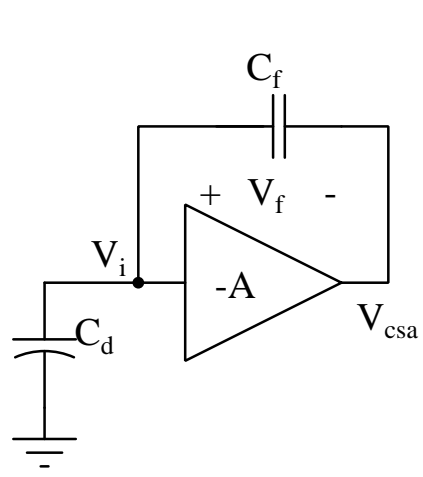

(a)

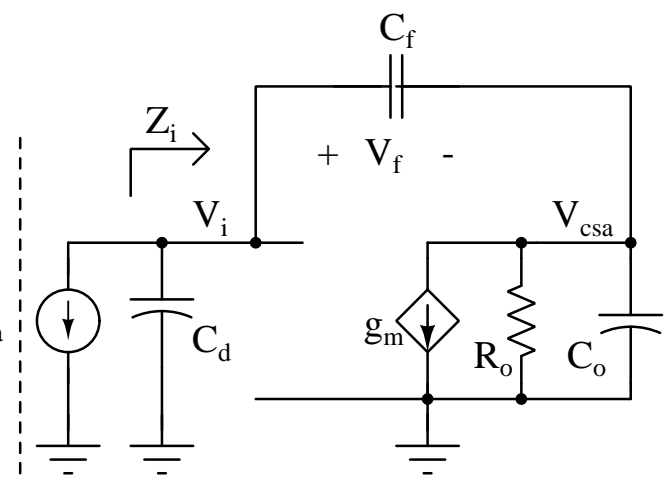

(b)

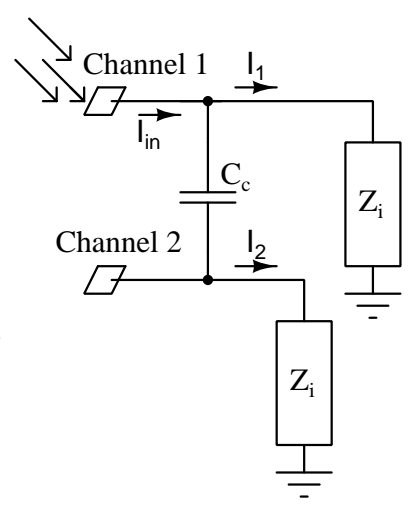

(c)

Figure 3.2: (a) Simple model of the CSA for the CSA open loop gain estimation; (b) Dominant pole model of the CSA; (c) Circuit equivalent to estimate the crosstalk between adjacent channels. 
difference across $C_{f}$ is given by $V_{f}=V_{i}-(-\mathrm{A}) V_{i}=(\mathrm{A}+1) V_{i}$ where $V_{i}$ is the voltage at the CSA input node. Considering the input charge $\left(Q_{i}\right)$ is completely transferred to the $C_{f}$ capacitor $\left(Q_{i}=Q_{f}\right)$, the effective input capacitance is given by [SPIELER, 2003]:

$$
C_{i}=\frac{Q_{i}}{V_{i}}=C_{f}(1+A) \text { then } V_{i}=\frac{Q_{i}}{C_{f}(1+A)}
$$

The CSA gain $\left(A_{Q}\right)$ is defined by:

$$
A_{Q}=\frac{d V_{c s a}}{d V_{i}}=\frac{A \cdot V_{i}}{C_{i} \cdot V_{i}}=\frac{A}{C_{i}}=\frac{A}{(1+A) C_{f}}
$$

if $A \gg 1$ then:

$$
A_{Q}=\frac{A}{(1+A) C f} \approx \frac{1}{C f}
$$

if $\mathrm{A}$ is high enough the CSA gain is controlled by the $C_{f}$ capacitance. $60 \mathrm{~dB}$ of open loop gain for the CSA design will set $0.1 \%$ of error on the calculated value with the equation 3.3.

\subsubsection{Residual charge}

The CSA gain was calculated considering that the input charge $Q_{i}$ is completely transferred to the $C_{f}$ integrator capacitor. However, a charge component remains on the detector capacitor $C_{d}\left(Q_{d}\right)$. The transferred charge percentage can be calculated by [SPIELER, 2003]:

$$
\frac{Q_{i}}{Q_{d}+Q_{i}}=\frac{C_{i} V_{i}}{Q_{d}+Q_{i}}=\frac{1}{1+\frac{C_{d}}{C_{i}}}
$$

The input capacitance should be much greater than $C_{d}$ for high charge transfer efficiency.

\subsubsection{Input impedance}

To estimate the input impedance requirement of the CSA, the dominant pole model shown in Figure 3.2b will be used instead of the simple open loop amplifier model shown in Figure 3.2a. This model assumes that the amplifier is an ideal operation transconductance amplifier (OTA). The open loop gain $\left(A_{v}\right)$ is expressed by:

$$
A_{v}=\frac{A}{1+\frac{j \cdot \omega}{\omega_{0}}}
$$


where $A=g_{m 1} \cdot R_{O}$ is the DC gain, $\omega_{O}=\frac{1}{R_{O} C_{O}}, R_{O}$ is the dominant pole resistance, $C_{O}$ is the dominant pole capacitance. In this model, the Gain-Bandwidth product (GBW) is given by [JOHNS; MARTIN, 2008]:

$$
G B W=A \cdot \omega_{0}
$$

The input impedance $\left(Z_{i}\right)$ is given by:

$$
Z_{i}=\frac{Z_{f}}{A_{v}+1}=\approx \frac{Z_{f}}{A_{v}}
$$

where $Z_{f}$ is the feedback impedance. For low frequency $A_{v}=A$ is constant then $Z i=\frac{-j}{w \cdot C_{f} \cdot A}$ (purely reactive). For high frequency $\left(w \gg \omega_{0}\right) A_{v}=-j \frac{A \cdot \omega_{0}}{w}$ then:

$$
Z_{i}=\frac{-j}{\omega C_{f}} \frac{1}{-j \frac{A \cdot \omega_{0}}{\omega}}=\frac{1}{A \cdot \omega_{o} C_{f}}=\frac{1}{G B W \cdot C_{f}}
$$

The amplifier operates in 90 degrees shift regime $\left(A_{v}=-j \frac{A \cdot \omega_{0}}{\omega}\right)$ during the CSA operation where the input impedance is resistive according to the equation (3.8). Low input impedance is necessary for low crosstalk between channels. The crosstalk between adjacent channels can be estimated by the equivalent circuit presented in Figure 3.2c. A coupling capacitance $\left(C_{c}\right)$ connected between the channels input is modeled as a impedance $Z_{c}=\frac{-j}{\omega C_{c}}$. The crosstalk value is calculated from the definition and it is given by:

$$
\text { Crosstalk }=20 \cdot \log _{10}\left(\frac{I_{2}}{I_{1}}\right)=20 \cdot \log _{10}\left(\frac{Z_{i}}{Z_{c}+Z_{i}}\right)
$$

Figure 3.3 presents crosstalk versus frequency obtained from equation 3.9 for $A=40 \mathrm{~dB}$, $\mathrm{GBW}=2 \mathrm{GHz}, C_{c}=1 p F$ and $C_{f}=1 p F$. According this results, a readout front-end composed of a $160 \mathrm{~ns}$ 4th order pulse shaper $\left(w \approx 1 / \tau_{0} \cdot \sqrt{2^{1 / n}-1}\right.$ is the cut-off frequency for cascaded $n$-stages low pass-filter) and a CSA with the above specifications will present $-52 \mathrm{~dB}(0.2 \%)$ of crosstalk according to equation 3.9.

\subsubsection{Frequency response}

The first step of the design is to study the simplified small signal model of the amplifier which is shown in Figure 3.1, where $r_{o 1}, r_{M B 2}, r_{o C}, r_{O M B 3}, r_{O M B 5}$ represent the output resistance of the transistors $M_{1}, M_{B 2}, M_{C}, M_{B 3}$ and $M_{B 5}$, respectively, $g_{m C}$ 


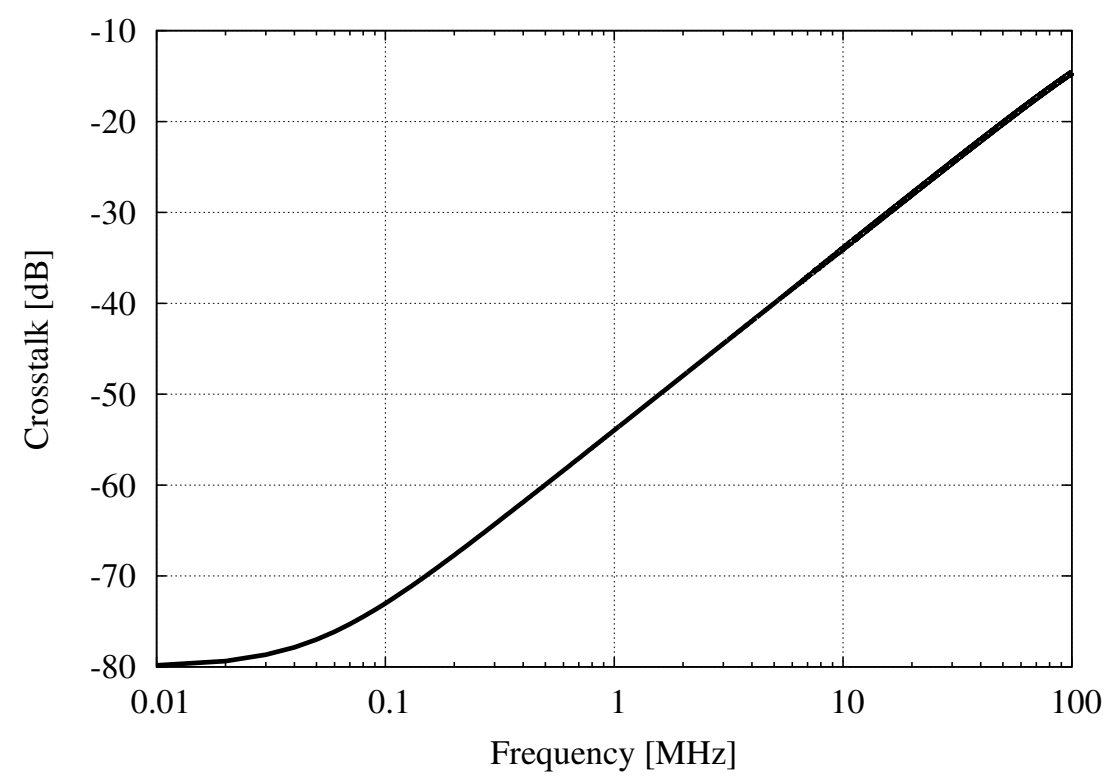

Figure 3.3: Crosstalk versus frequency obtained from equation 3.9 for $A=40 \mathrm{~dB}, \mathrm{GBW}=2 \mathrm{GHz}$, $C_{c}=1 p F$ and $C_{f}=1 p F$

is the transconductance of the transistor $M_{C}$ and $C_{2}$ and $C_{3}$ are the total capacitance associated to the node 2 and 3 , respectively. The transconductance of the amplifier is given by $g_{m 1}$ of the input transistor $M_{1}$. The open loop gain of the amplifier $(A)$ from the small signal model can be approximated by:

$$
A \approx-g_{m 1}\left(r_{o 1} \| r_{o M B 2}\right) g_{m C}\left(r_{o C} \| r_{o M B 3}\right)
$$

Commonly, the position of the dominant pole $\left(p_{1}\right)$ is determinate by $\left(C_{3} r_{O M B 3} \| r_{O C}\right)^{-1}$. The non-dominant pole is given by $\left(C_{2} r_{o 1} \| R_{i n 2}\right)^{-1}\left(p_{2}\right)$ in which $R_{i n 2}$ is the input resistance on the $M_{C}$ source. $C_{2}$ is composed by the $C_{g s C}$ capacitances of $M_{C}, C_{g d M B 2}$ of $M_{B 2}$ and $C_{d b 1}$ and $C_{g d 1}$ capacitances of $M_{1}$. However, in order to obtain a low noise design the dimensions of the input transistor can be quite large. As a results, the pole generated by the drain-bulk capacitance $C_{d b 1}$ will be close to the dominant pole. A compensation capacitor $C_{c}$ connected between nodes 2 and 3 is included to compensate the lower frequency pole $p_{2}$ by generating a zero in the transfer function [SANSEN; CHANG, 1990a].

\subsubsection{ENC}

To calculate the total value of ENC of the a readout system, the front-end noise model for a Semi-Gaussian shaper shown in Figure 3.4 is used, where $v_{i a}^{2}$ represents the input-refered voltage noise of the amplfier. The main $v_{i a}^{2}$ noise contributions are the 


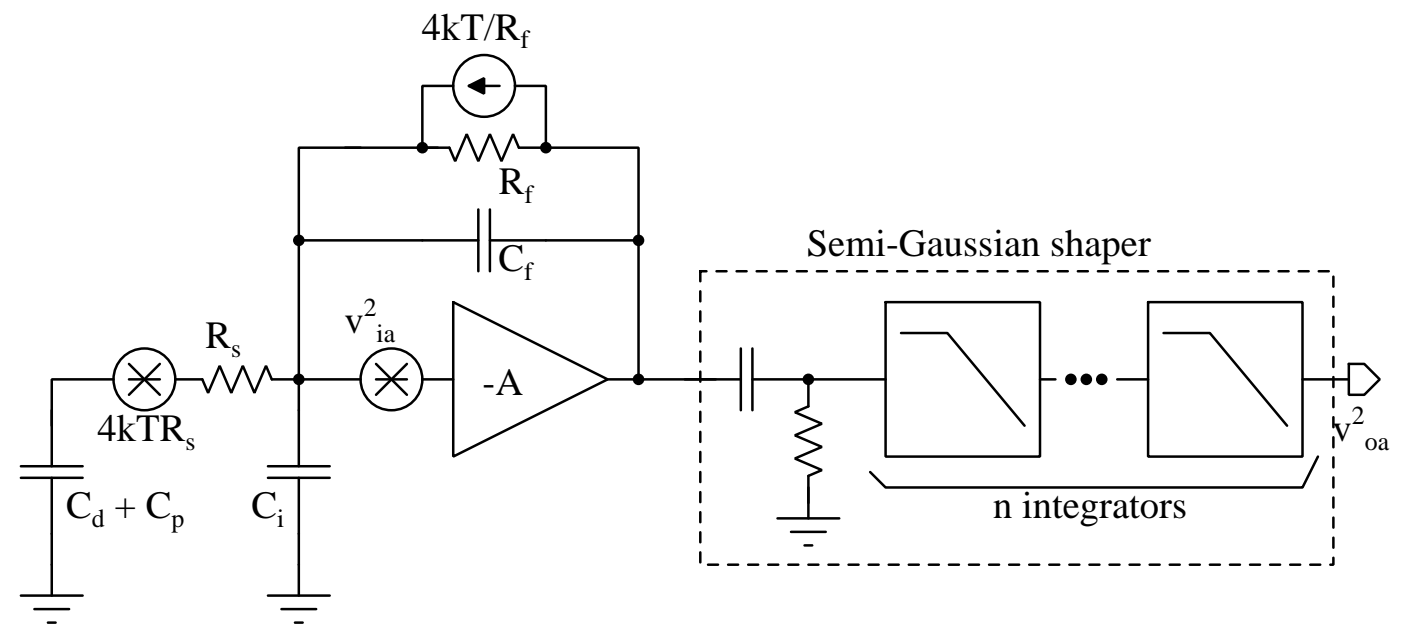

Figure 3.4: Front-end noise model for a Semi-Gaussian shaper.

channel thermal noise and the flicker noise of the amplifier input transisor $M_{1}$. However, the noise generated by the bias current mirror can have significant contribution specially when the supply voltage is low. In this model the detector is represented by the capacitance $C_{d}$, the $R_{f}$ is the feedback resistance of the CSA and $C_{i}$ is the input capacitance of the CSA. $C_{p}$ and $R_{s}$ represent the parasitic capacitance and the series resistance of the interconnection between the detector and the amplifier input respectively. It can be easily shown that the total noise power spectrum at the output of the CSA from circuit analyzing is given by [CHONG; SANSEN, 1991]:

$$
v_{o a}^{2}(s)=\left|\frac{C_{d}+C_{p}+C_{f}+C_{i}}{C_{f}}\right|^{2} v_{i a}^{2}
$$

In order to calculate the ENC, the total RMS noise at the shaper output must be calculated. The transfer function of the Semi-Gaussian shaper is given by equation 2.1 which is construed by one RC differentiator and $n$ integrators. The output noise spectrum is weighted by the transfer function of the shaper and then the total integrated RMS noise is thus given by:

$$
v_{\text {tot }}^{2}=\int_{0}^{\infty}\left|v_{o a}(j 2 \pi f)\right|^{2}|H(j 2 \pi f)|^{2} d f
$$

Its is important to note that the total noise power spectrum $v_{o a}^{2}(s)$ in equation (3.12) comprises several independent noise components. Therefore, the above integral can be calculated for each component separately. 


\section{ENC due to $M_{1}$ channel thermal noise:}

The noise component due to the channel thermal noise of the CSA input transistor is given by [TSIVIDIS, 1987]:

$$
\left.v_{i a}^{2}\right|_{c h}=4 k T n_{s} \gamma \cdot \frac{1}{g_{m 1}}
$$

where $n_{S}$ is the sub-threshold slope factor and $\gamma$ is a constant that depend of the operation region: $2 / 3$ for strong inversion and $1 / 2$ for weak inversionn. $g_{m 1}$ is the transconductance of the CSA input transistor and it depends of the operation region.

Combining the above equation with (3.11) and the general expression (3.12), the total integrated RMS thermal noise becomes explicitly given by:

$$
\left.v_{o a}^{2}\right|_{c h}=\frac{4 k T n_{s} \gamma}{g_{m 1}} \cdot\left(\frac{C_{t}}{C_{f}}\right)^{2} \cdot \frac{A^{2 n} B\left(\frac{3}{2}, n-\frac{1}{2}\right) n}{4 \pi \tau_{s}}
$$

where $B(x, y)$ is the Beta-function, $\tau_{s}$ is the peaking time of the Semi-Gaussian pulse shaper which is defined as $\tau_{s}=n \tau_{o}$, and $C_{t}$ is the total capacitance at the input node of the CSA $\left(C_{d}+C_{p}+C_{f}+C_{i}\right)$. Dividing the above expression by the signal amplitude due to one electron charge (equation 2.2) the $\mathrm{ENC}_{c h}$ due to the channel thermal noise is obtained:

$$
E N C_{c h}^{2}=\frac{4 k T n_{s} \gamma}{g_{m 1}} \cdot \frac{C_{t}^{2} B\left(\frac{3}{2}, n-\frac{1}{2}\right) n}{q^{2} 4 \pi \tau_{s}} \cdot \frac{n !^{2} e^{2 n}}{n^{2 n}}
$$

Considering the factor $D_{c h}=4 k T n_{s} \gamma \cdot \frac{B\left(\frac{3}{2}, n-\frac{1}{2}\right) n}{q^{2} 4 \pi} \cdot \frac{n !^{2} e^{2 n}}{n^{2 n}}$ a constant for a specific shaper order, the $\mathrm{ENC}_{c h}$ expression is as follows:

$$
E N C_{c h}^{2}=\frac{D_{c h} C_{t}^{2}}{g_{m 1} \tau_{s}}
$$

From the above equation some conclusions can directly be drawn [CHONG; SANSEN, 1991]:

- CSA must be designed to have an as large as possible transconductance $g_{m 1}$ and as low as possible total input capacitance $C_{t}$.

- Large peaking time $\tau_{s}$ has the effect of limiting the thermal noise contribution.

- $E N C_{c h}$ is totally independent of the dc gain of the pulse shaper. 
ENC due to $M_{1}$ flicker $(1 / f)$ noise:

CMOS technology has a high component of $1 / f$ noise and its effect on the detector resolution must be studied. The $1 / f$ noise source of the input MOS transistor of the CSA is given by [CHONG; SANSEN, 1991]:

$$
\left.v_{i a}^{2}\right|_{1 / f}=\frac{K_{f}}{C_{o x}^{2} W L f}
$$

where $K_{f}$ is the flicker noise coefficient and $f$ is the frequency. Substituting the above equation in the equations (3.11) and (3.12) the RMS output $1 / f$ noise can be obtained:

$$
\left.v_{o a}^{2}\right|_{1 / f}=\frac{K_{f}}{C_{o x}^{2} W L} \cdot\left(\frac{C_{t}}{C_{f}}\right)^{2} \cdot \frac{A^{2 n}}{2 n}
$$

The $E N C_{1 / f}$ is calculated as the ratio of the above equation and the equation (2.2):

$$
E N C_{1 / f}^{2}=\frac{K_{f}}{C_{o x}^{2} W L} \cdot \frac{C_{t}^{2}}{q^{2} 2 n} \cdot \frac{n !^{2} e^{2 n}}{n^{2 n}}
$$

Considering the factor $D_{f}=\frac{K_{f}}{C_{o x}^{2}} \cdot \frac{1}{q^{2} 2 n} \cdot \frac{n !^{2} e^{2 n}}{n^{2 n}}$ a constant for a specific shaper order, the $E N C_{1 / f}$ expression is as follows:

$$
E N C_{1 / f}^{2}=\frac{D_{f} C_{t}^{2}}{W L}
$$

Important conclusions taken from the $E N C_{1 / f}$ equation are:

- The flicker noise contribution is totally independent of the peaking time of the shaper, but it depends slightly on the number of the integrators in the pulse shaper.

- $K_{f}, C_{O x}$ and the input transistors dimensions affect strongly the contribution of the $1 / f$ noise.

\section{ENC due to the transistors of the bias current mirror}

The noise model of the CSA with the noise contribution from each transistor of the circuit less $M_{1}$ is presented in Figure ??. Each current/voltage noise in this equivalent circuit is identify with the sub-index of the element/device that generates such noise. 


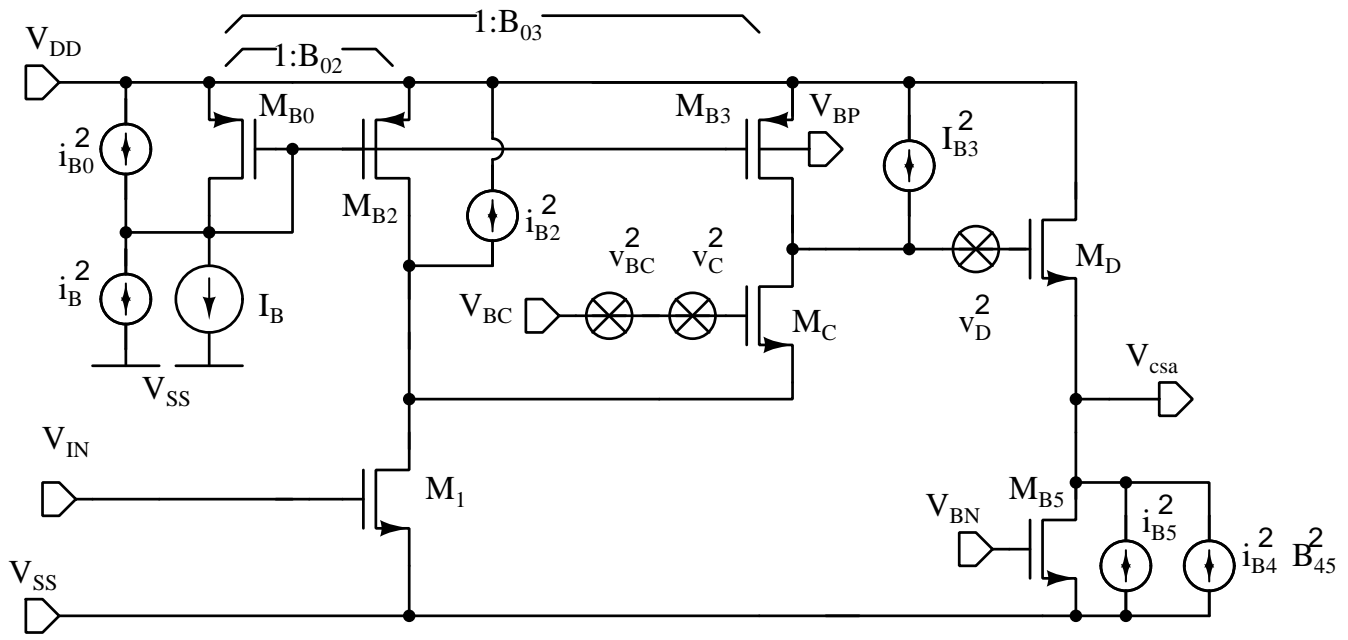

Figure 3.5: Equivalent circuit for noise analysis of the bias current mirror.

For example, $I_{B 0}^{2}$ is the current noise resulting from $M_{B 0}$ and $I_{B}^{2}$ is the current noise resulting from the bias current source $\left(I_{B}\right) . B_{02}, B_{45}$ represent the copy current factor between the current mirror transistors $M_{B 0}-M_{B 2}, M_{B 4^{-}} M_{B 5}$ and $M_{B 4}{ }^{-M_{B 5}}$ respectively. An expression of total refereed to the input noise resulting from the bias current mirror transistors $\left(v_{B}^{2}\right)$ can be determinate by circuit analysis of the presented noise model as follows:

$$
\begin{aligned}
v_{B}^{2}= & i_{B}^{2} \frac{B_{02}^{2}}{g_{m 1}^{2}}+i_{B 0}^{2} \frac{B_{02}^{2}}{g_{m 1}^{2}}+i_{B 2}^{2} \frac{B_{02}^{2}}{g_{m 1}^{2}}+i_{B 3}^{2} \frac{B_{03}^{2}}{g_{m 1}^{2}}+i_{B 4}^{2} \frac{B_{45}^{2}}{g_{m D}^{2} A_{v}^{2}}+i_{B 5}^{2} \frac{1}{g_{m D}^{2} A_{v}^{2}} \\
& +v_{B C}^{2} \frac{1}{\left(g_{m 1} r_{o 12}\right)^{2}}+v_{C}^{2} \frac{1}{\left(g_{m 1} r_{o 12}\right)^{2}}+v_{D}^{2} \frac{1}{A_{v}^{2}}+i_{B 5}^{2} \frac{1}{g_{m B 5}^{2} A_{v}^{2}}+i_{B 4}^{2} \frac{B_{45}^{2}}{g_{m B 4}^{2} A_{v}^{2}}
\end{aligned}
$$

where $r_{O 12}$ is the output resistance of the first stage. The noise components from $M_{B 5}$ and $M_{B 4}$ can be disconsidered because they are divided by the amplifier open loop gain. Similarly, other noise contributions that we can disconsider in this analysis are the $M_{D}$ and $M_{C}$ which are divided by the first stage gain. In this way, the previous equation is follows as:

$$
v_{B}^{2}=i_{B}^{2} \frac{B_{02}^{2}+B_{03}^{2}}{g_{m 1}^{2}}+i_{B 0}^{2} \frac{B_{02}^{2}+B_{03}^{2}}{g_{m 1}^{2}}+i_{B 2}^{2} \frac{1}{g_{m 1}^{2}}+i_{B 3}^{2} \frac{1}{g_{m 1}^{2}}
$$

Then we can calculate the ENC contribution of the bias current mirror in the same way as $M_{1}$ contribution was calculate:

$$
E N C_{B 0}^{2}=\left[\frac{D_{c h p} C_{t}^{2}}{g_{m B 0} \tau_{s}}+\frac{D_{f p} C_{t}^{2}}{(W L)_{B 0}}\right] \frac{B_{02}}{g_{m 1}^{2}}
$$




$$
\begin{aligned}
& E N C_{B 2}^{2}=\left[\frac{D_{c h p} C_{t}^{2}}{g_{m B 2} \tau_{s}}+\frac{D_{f p} C_{t}^{2}}{(W L)_{B 2}}\right] \frac{1}{g_{m 1}^{2}} \\
& E N C_{B 3}^{2}=\left[\frac{D_{c h p} C_{t}^{2}}{g_{m B 3} \tau_{s}}+\frac{D_{f p} C_{t}^{2}}{(W L)_{B 3}}\right] \frac{1}{g_{m 1}^{2}} \\
& E N C_{B}^{2}=\left[\frac{D_{c h n} C_{t}^{2}}{g_{m B} \tau_{s}}+\frac{D_{f n} C_{t}^{2}}{(W L)_{B}}\right] \frac{1}{g_{m 1}^{2}}
\end{aligned}
$$

where $D_{c h p}, D_{c h n}, D_{f n}$ and $D_{f p}$ are the PMOS-NMOS cases of the $D_{c h}$ and $D_{f}$ constants previously defined. $g_{m B}$ and $(W L)_{B}$ are the transconductance and dimension of the output transistor of the bias current circuit generator (NMOS). Important design recommendations taken from the ENC bias current mirror equations are:

- $g_{m 1}$ the transconductance of the bias current mirror transistors $\left(g_{m B 0}, g_{m B 2}\right.$ and $\left.g_{m B 3}\right)$ must be maximized for noise minimizing.

- The copy current factors $B_{02}$ and $B_{03}$ must be maximized for noise minimizing.

The contribution of bias current mirror noise components to the total ENC can be disconsidered if decoupling capacitance on $V_{B P}, V_{B N}$ and $V_{B C}$ are included [SOLTVEIT et al., 2012].

ENC due to $R_{s}$ and $R_{f}$ :

The ENC components due the series and the feedback resistance of the CSA can be calculated from the noise model presented in Figure 3.4 using the same proceeding used for the channel resistance and $1 / f$ ENC components. $E N C_{r s}$ and $E N C_{r f}$ are given by:

$$
\begin{gathered}
E N C_{r s}^{2}=4 k T \cdot R_{s} \cdot \frac{C_{t}^{2} B\left(\frac{3}{2}, n-\frac{1}{2}\right) n}{q^{2} 4 \pi \tau_{s}} \cdot \frac{n !^{2} e^{2 n}}{n^{2 n}} \\
E N C_{r f}^{2}=\frac{4 k T}{R_{f}} \cdot \tau_{s} \cdot \frac{B\left(\frac{1}{2}, n+\frac{1}{2}\right)}{q^{2} 4 \pi n} \cdot \frac{n !^{2} e^{2 n}}{n^{2 n}}
\end{gathered}
$$

Since the four ENCs components calculated from four independent noise sources, the total equivalent noise charge $E N C_{T}$ is simply given by the sum of squares of the four components:

$$
E N C_{T}=\sqrt{E N C_{c h}^{2}+E N C_{1 / f}^{2}+E N C_{r s}^{2}+E N C_{r f}^{2}}
$$

In the derivation of the referred input noise of the CSA only the input transistor noise is taken into account. However, extra ENC components due to the CSA current sources 


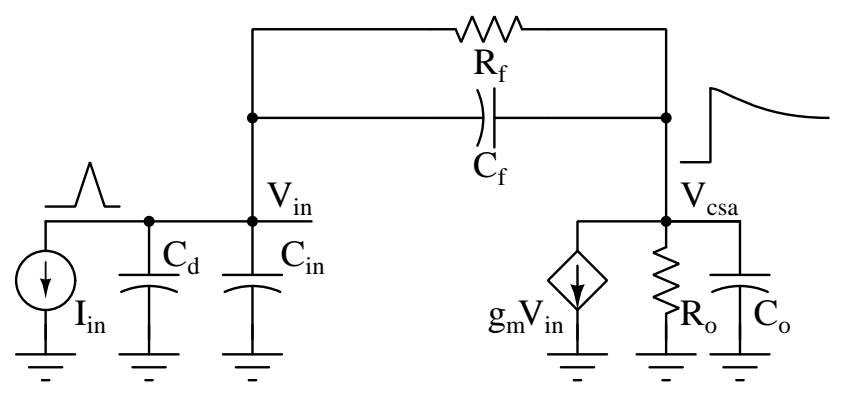

Figure 3.6: Block schematic of the CSA transient model

are present in addition to the noise components due to input transistor. The extra noise components should be kept negligibly smaller than the channel thermal noise and the flicker noise components. MOS transistors exhibit also parasitic noise due to the resistive poly gate and the distributed substrate resistance. These noise contributions can prevail over the channel thermal noise if no special caution is taken, but they can be minimize by performing an appropriate layout of the amplifier, especially of the input transistor [JOHNS; MARTIN, 2008].

\subsubsection{Rise time of the impulse response}

For the transient analysis, the block schematic of the CSA shown in Figure 3.6 is used. The resistive feedback is provided by a large-value resistor $R_{f}$ in parallel with the feedback capacitance $C_{f}$. Assume the amplifier is an OTA with one dominant pole described by equation 3.5. The transfer function is given by [CHONG; SANSEN, 1991]:

$$
\frac{V_{c s a}(s)}{I_{i n}(s)}=\frac{g_{m 1}}{g_{m 1} / R_{f}+s g_{m 1} C_{f}+s^{2} C_{t}\left(C_{f}+C_{O}\right)}
$$

where it is assume that $g_{m 1} R_{O} \gg 1$ and $g_{m 1} R_{f} \gg 1$ (the OTA the output impedance is always very high and the feedback resistor $R_{f}$ is also of a high value). Under the assumption that the two poles are widely spread, the pole position are given by:

$$
\begin{gathered}
p_{1}=\frac{1}{2 \pi R_{f} C_{f}}=\frac{1}{\tau_{1}} \\
p_{2}=\frac{g_{m 1} C_{f}}{2 \pi C_{t}\left(C_{O}+C_{f}\right)}=\frac{G B W C_{f}}{C_{t}}=\frac{1}{\tau_{2}}
\end{gathered}
$$

The first pole is due to the feedback components and the second pole is the result of the feedback capacitance. Since the input current can be approximated as a Dirac impulse with an integrated area of $\mathrm{Q}$, the time response $V_{c s a}(t)$ can be obtained by 

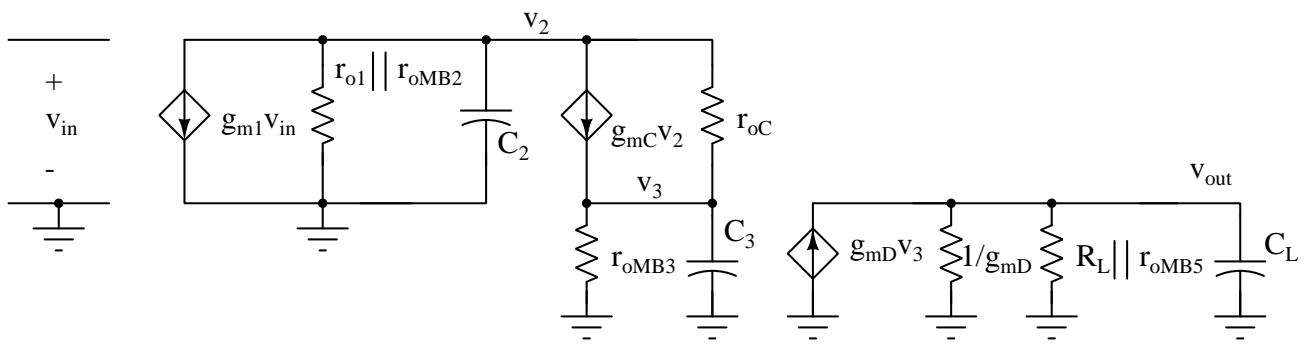

Figure 3.7: Small signal model of the cascode amplifier for the open loop gain estimation taking the inverse Laplace transformation of the transfer function (3.30). Therefore, the output signal in the time domain is given by:

$$
V_{c s a}(t)=\frac{Q \tau_{1}}{C_{f}\left(\tau_{1}-\tau_{2}\right)}\left(e^{-t / \tau_{1}}-e^{-t / \tau_{2}}\right)
$$

Since in all cases $\tau_{1} \gg \tau_{2}$, the above equation represents an exponential rising step signal governed by GBW and a slowly decaying rail governed by the feedback resistance. The rise time of the step signal $t_{r}$ is defined as the time interval between which the output signal rises from $10 \%$ to $90 \%$ of the amplitude. It is calculated by [CHONG; SANSEN, 1991]:

$$
t_{r}=2.2 \tau_{2}=2.2 \frac{C_{t}}{G B W C_{f}}
$$

It is seen from the above equation that for a given detector capacitance the rise time can be minimized by increasing the GBW of the OTA and using a large feedback capacitance.

\subsection{CSA Design}

In order to obtain a low noise CSA, reaching a GBW high enough for crosstalk lower than $0.2 \%$ and a fast impulse response lower than $1 \mathrm{~ns}$, some considerations must be taken into account for the design.

\subsubsection{Feedback capacitance selection}

An adequate value of feedback resistance should be chosen according to the input dynamic range requirement. The input dynamic range for gaseous detectors readout front-end is defined in charge unit (Coulombs). As mentioned in the introduction chapter, the dynamic range for the ALICE TPC should be configurable for $100 \mathrm{fC}$ and $67 \mathrm{fC}$ and for $\mathrm{MCH}$ the input dynamic range specification is $500 \mathrm{fC}$ which is the worst case 
$\left(Q_{\max }\right)$. The input dynamic range of the CSA is limited by the output dynamic range of the amplifier. The maximum output voltage of the amplifier is limited by the saturation condition of the $M_{B 3}$ transistor:

$$
V_{\text {out }}^{\max }=V_{D D}-V_{\text {sat }} M_{B 3}
$$

where $V_{s a t} M_{B 3}$ is the saturation voltage of the $M_{B 3}$ transistor. The minimum output voltage of the amplifier is limited by the saturation condition of the $M_{B 5}$ :

$$
V_{\text {out }}^{\text {min }}=V_{\text {sat }} M_{B 5}
$$

where $V_{\text {sat }} M_{B 5}$ is the saturation voltage of the $M_{B 5}$ transistor. The maximum amplitude of the CSA step output signal is defined by the CSA gain and the maximum input charge:

$$
\Delta V_{\text {out }}=\frac{Q_{\max }}{C_{f}}
$$

The $M_{B 1}-M_{B 5}$ transistors should operate in strong inversion to minimize them noise contribution to the total system noise [CHONG; SANSEN, 1991]. A good feedback capacitance value that provides a good trade-off between noise, sensitivity and the worst case of the input dynamic range (500fC) is $3 p F$. According to the configuration case, the CSA feedback capacitance will be adjusted for $t_{S}=160 \mathrm{~ns}$ case $\left(3 \mathrm{pF} / k_{1}\right.$ where $\left.k_{1}=300 \mathrm{~ns} / 160 \mathrm{~ns}\right)$ and $t_{s}=80 \mathrm{~ns}$ case $\left(3 p F / k_{2}\right.$ where $\left.k_{2}=300 \mathrm{~ns} / 80 \mathrm{~ns}\right)$ via transmission gates.

\subsubsection{GBW selection}

The GBW of the CSA amplifier has two selection criteria, the input impedance requirement and the rise time of the impulse response requirement. The higher value within the required for each specification should be selected for the amplifier design. The input impedance of the CSA can be calculated from equation (3.9) for $w \approx 1 / \tau_{0} \cdot \sqrt{2^{1 / n}-1}$ (central frequency of the Semi-Gaussian pulse shaper) and the crosstalk specification. The coupling capacitance between adjacent channels inputs was estimated to be $2 \mathrm{pF}$ (value provided by the TPC electronic group). It is considered in this capacitance the package and board parasitic coupling capacitances. The GBW requirement for $1 \mathrm{~ns}$ of rise time of the impulsive response can be estimated from equation (3.34). The 
Table 3.1: Summary of the obtained GBW values according to the input impedance and rise time requirements

\begin{tabular}{|c|c|c|c|}
\hline Variable & $\mathbf{T C P} @ t_{s}=80 \mathrm{~ns}$ & $\mathbf{T C P} @ t_{s}=160 \mathrm{~ns}$ & $\mathrm{MCH}$ \\
\hline$t_{0}\left(t_{S} / n\right)$ & $20 \mathrm{~ns}$ & $40 \mathrm{~ns}$ & $80 \mathrm{~ns}$ \\
\hline$C_{d}$ & $18.5 \mathrm{pF}$ & $18.5 \mathrm{pF}$ & $40 \mathrm{pF}$ \\
\hline Crosstalk & $0.3 \%$ & $0.2 \%$ & $0.2 \%$ \\
\hline$Z_{c}$ & $23 \mathrm{~K} \Omega$ & $46 K \Omega$ & $92 \mathrm{~K} \Omega$ \\
\hline$R_{s}{ }^{*}$ & $70 \Omega$ & $70 \Omega$ & $70 \Omega$ \\
\hline$Z_{i}^{* *}$ & $84 \Omega$ & $45 \Omega$ & $237 \Omega$ \\
\hline$G B W$ (imput impedance) & $4.35 \mathrm{GHz}$ & $1.2 \mathrm{GHz}$ & $210 \mathrm{MHz}$ \\
\hline$G B W$ (rise time) & $430 \mathrm{MHz}$ & $430 \mathrm{MHz}$ & $1.9 \mathrm{GHz}$ \\
\hline
\end{tabular}

obtained GBW values for TPC and MCH cases are summarized in Table 3.1.

The GBW worst case from Table 3.1 is the $t_{s}=80$ ns case $(\mathrm{GBW}=4.53 \mathrm{GHz})$. The amplifier transconductance can be calculated from the GBW and the feedback capacitance $\left(C_{f}=800 \mathrm{fF}\right.$ for the case of $80 \mathrm{~ns}$ of peaking time) by $g_{m}=\cdot G B W \cdot C_{f}=21.6 \mathrm{mS}$.

\subsubsection{Input transistor design}

The amplifier transconductance (the CSA amplifier transconductance is defined by the input transistor) required by ENC specification can be calculate from the derived equations for each noise components. From the given specification three parameters are fixed: the peaking time, the shaper order and the detector capacitance. The amplifier transconductance and the total input capacitance $\left(C_{t}\right)$ have a non-linear dependence with the input transistors dimensions. These depend on the operation region of the input transistor: weak inversion, moderate inversion, strong inversion or saturation velocity [ENZ et al., 1995]. The level 1 model equations do not consider the physics effects when the transistor operates in a different operating region of the strong inversion. A optimum value of transconductance for the input transistor can be chosen if continuous equations valid in all operating regions are used. The equations presented by Yannis Tsividis [TSIVIDIS, 1987] meet this characteristic, and therefore they were utilized. These equations and the transistor parameters of the TSMC 130nm CMOS technology used in the following study are described in Table 3.2. 
Table 3.2: Continuous equations (model) valid in all regions of operation taken from [TSIVIDIS, 1987]

\begin{tabular}{|c|c|}
\hline$\phi_{t}=\frac{k T}{q} \approx 26 m V\left(\mathrm{~T}=20^{\circ} \mathrm{C}\right)$ & Thermal voltage \\
\hline$\beta=\mu_{n} C_{O x} \cdot \frac{W}{L}$ & MOS transistor gain factor \\
\hline$I_{z}=2 n \beta \phi_{t}^{2}$ & Characteristic current \\
\hline$I_{d}=I_{z} \cdot \ln ^{2}\left[1+e^{\frac{V_{g s}-V_{t}}{2 n \phi_{t}^{2}}}\right]$ & Drain current \\
\hline $\mathrm{u}=I_{d} / I_{z}$ & Inversion coefficient \\
\hline$f(u)=\frac{1}{2}(\sqrt{1+4 u}+1)$ & Interpolation function \\
\hline$g_{m}=\frac{I_{d}}{n \phi_{t}} \frac{1}{f(u)}$ & Transconductance \\
\hline$C_{g s}=W L C_{O x}\left[\frac{3}{2}+\frac{f(u)}{u}\right]^{-1}+W \cdot C_{O v}$ & Gate-source capacitance \\
\hline$C_{g b}=W L C_{O x} \frac{n-1}{n}\left[1-\left(\frac{3}{2}+\frac{f(u)}{u}\right)^{-1}\right]$ & Gate-bulk Capacitance \\
\hline$S_{i}=4 k T\left(\frac{1}{2}+\frac{1}{9} \frac{u}{f^{2}(u)}\right)$ & $\begin{array}{l}\text { Power spectral densities of the chan- } \\
\text { nel thermal noise }\end{array}$ \\
\hline$S_{f}=\frac{K_{f}}{C_{O x} W L f}$ & $\begin{array}{l}\text { Power spectral densities of the flicker } \\
\text { noise }\end{array}$ \\
\hline \multicolumn{2}{|c|}{ Technology parameters } \\
\hline NMOS & PMOS \\
\hline$C_{o v}=0.35 \mathrm{fF} / \mu \mathrm{m}$ & $C_{O v}=0.35 f F / \mu m$ \\
\hline$C_{O x}=8.5 f F / \mu^{2} m$ & $C_{O x}=8.5 f F / \mu^{2} m$ \\
\hline$K_{f}=3.16 e^{-27} \mathrm{~J} / \mathrm{m}^{2}$ & $K_{f}=5.69 e^{-27} \mathrm{~J} / \mathrm{m}^{2}$ \\
\hline$V_{t}=0.34 \mathrm{~V}$ & $V_{t}=-0.32 \mathrm{~V}$ \\
\hline$n=1.5$ & $n=1.5$ \\
\hline$\mu C_{O x}=275 \mu A / V^{2}$ & $\mu C_{o x}=85 \mu A / V^{2}$ \\
\hline
\end{tabular}

The CSA feedback resistance should be on the order of some $\mathrm{M} \Omega$ for low noise contribution but high values limit the front-end counting rate. A $3 M \Omega$ feedback resistance 
was chosen as a trade-off between these issues. The series resistance represents the resistance of the pad ESD protection. This resistance limits the crosstalk specification because it is in series with the CSA input impedance. A $70 \Omega$ resistance was chosen as trade-off between ESD protection efficiency and crosstalk. The ENC components due to $R_{f}$ is independent of the CSA input capacitance and it is fixed to $E N C_{r f}=139 \mathrm{e}$ $\left(t_{s}=160 \mathrm{~ns}\right.$ and $\left.t_{s}=80 \mathrm{~ns}\right)$ for TPC and $E N C_{r f}=197 \mathrm{e}$ for $\mathrm{MCH}$. An important extra noise contributor is the poly-gate of the transistor. Very wide transistors will present high poly-gate resistance. Its contribution is here minimized by dividing the input transistor in several strips. The final poly-gate resistance of the transistor will be the parallel of the $n_{f}$ strips resistance and it can be calculated by[CHONG; SANSEN, 1991]:

$$
R_{g}=\frac{R_{i}}{12 n_{f}}
$$

where $R_{i}$ is the resistance of the single poly-stripe of the width of the input transistor. This equation considers that the strips are also connected at both ends.

$E N C_{c h}, E N C_{f}$ and $E N C_{r s}$ depend on the gate capacitance, the transconductance and the operation region of the input transistor, so they were modeled by the continuous equation presented in Table 3.2 as a function of $\mathrm{W}$ and L. Figure 3.8 shows the ENC components $E N C_{c h}, E N C_{r s}, E N C_{r f}, E N C_{f}$ and the total ENC $\left(E N C_{t}\right)$ versus the channel width(W) of the CSA input transistor for $t_{s}=160 n s @ C_{d}=18.5 p F, t_{s}=80 n s @ C_{d}=$ $18.5 p F, t_{s}=320 n s @ C_{d}=40 p F$ and $I_{d}=1 m A$. The $E N C_{B 0}, E N C_{B 2}$ and $E N C_{B 3}$ contributions have been disconsidered for the input transistor design and they will be studied in a next section. The minimum channel length $\left(L=L_{\min }\right)$ was chosen to minimize the input capacitance of the CSA. Figure 3.9 shows $E N C_{t}$ calculation for each peaking time case for $I_{d}=250 \mu A, I_{d}=2 m A \mu A$ and $I_{d}=3 \mu A$. From Figure 3.8 anf 3.9 is possible highlight that $E N C_{t}$ has a minimum $\left(E N C_{\text {min }}\right)$ at $W_{\text {opt }}$ and this minimum has low dependency of $I_{d}$, i.e., we can assume that $W_{\text {opt }}$ does not depend on $I_{d}$. Figure 3.10 illustrates $E N C_{\min }$ versus $I_{d}$ for each peaking time case. From this graph is clear that above $I_{d}=2 m A$ the noise improvement with the drain current increasing is low. 


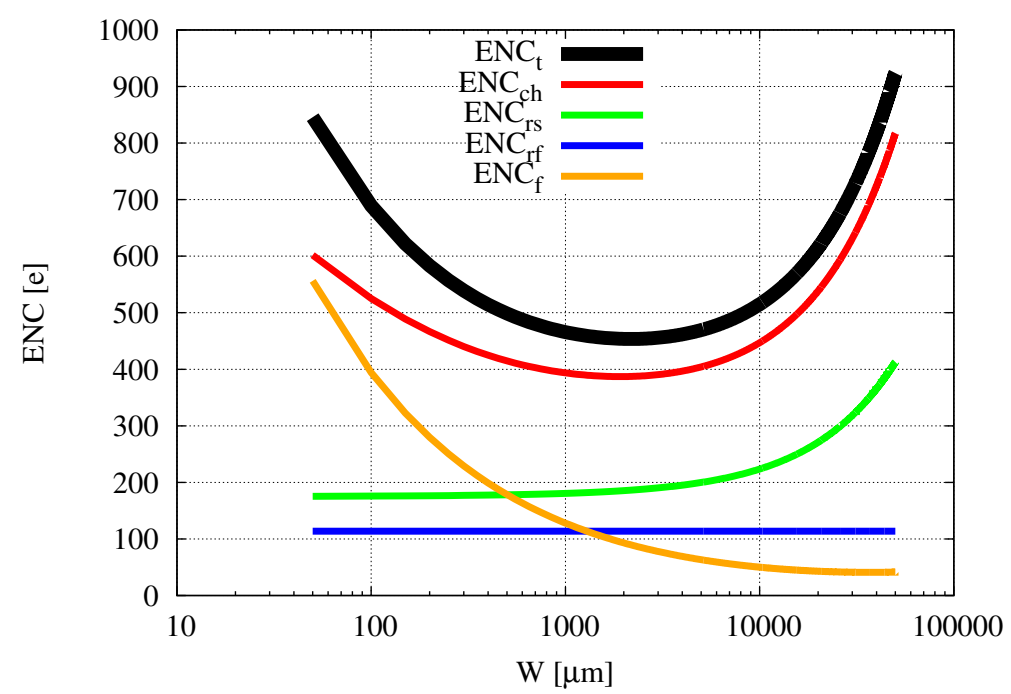

(a)

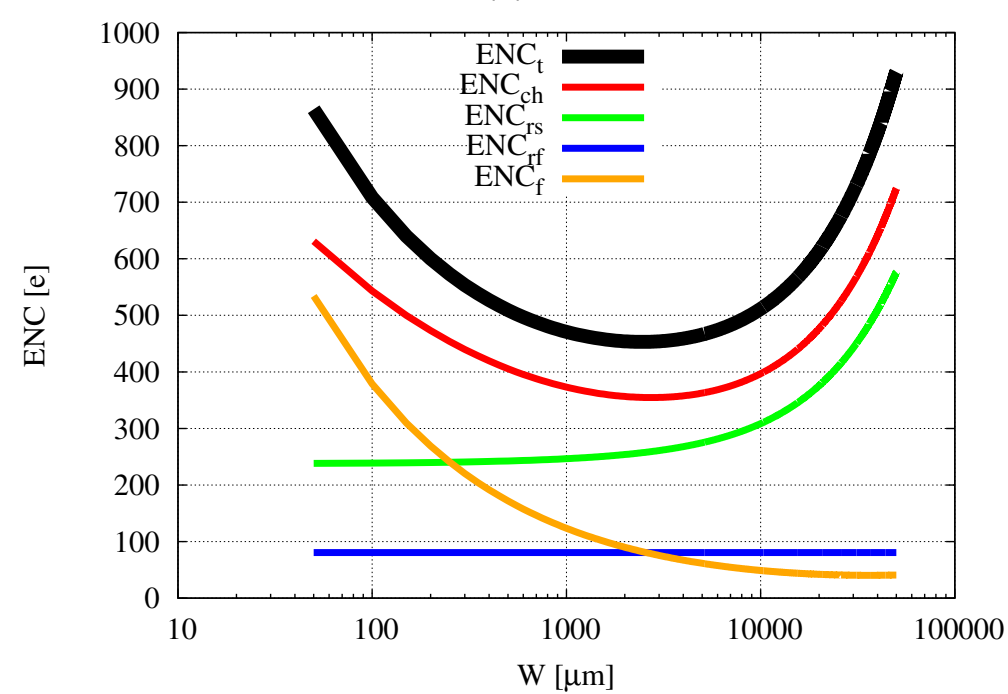

(b)

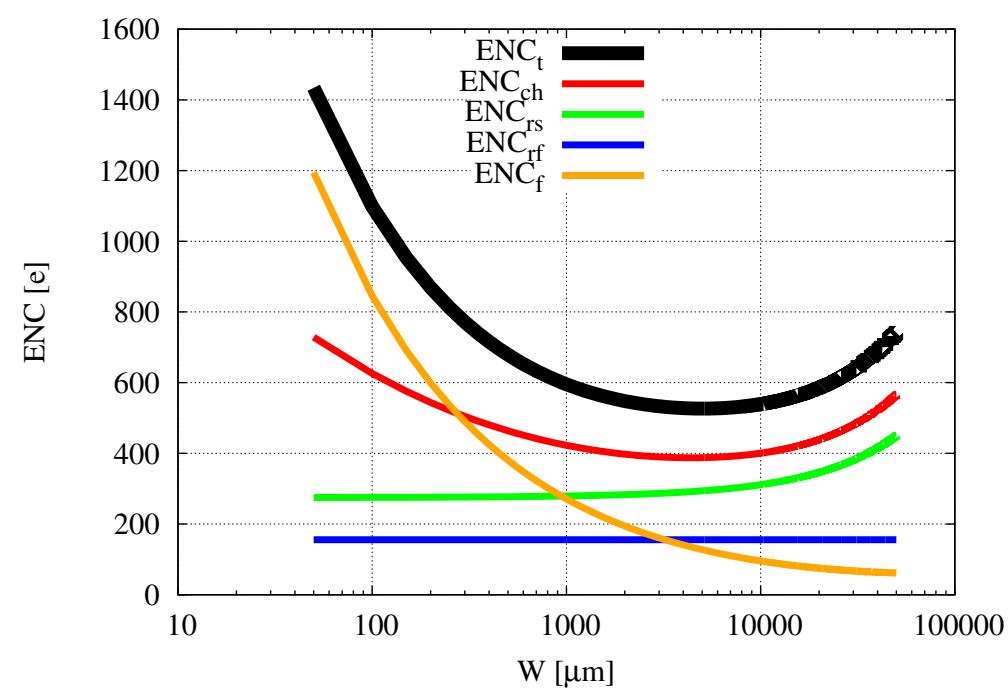

(c)

Figure 3.8: Theoretical ENC components versus the channel width (W) of the CSA input transistor for $I_{d}=1 \mathrm{~mA}, L=130 \mathrm{~nm}$ and (a) $t_{s}=160 \mathrm{~ns}, C_{d}=18.5 \mathrm{pF}$; (b) $t_{s}=80 \mathrm{~ns}, C_{d}=$ $18.5 \mathrm{pF}$; (c) $t_{s}=300 \mathrm{~ns}, C_{d}=40 \mathrm{pF}$. 


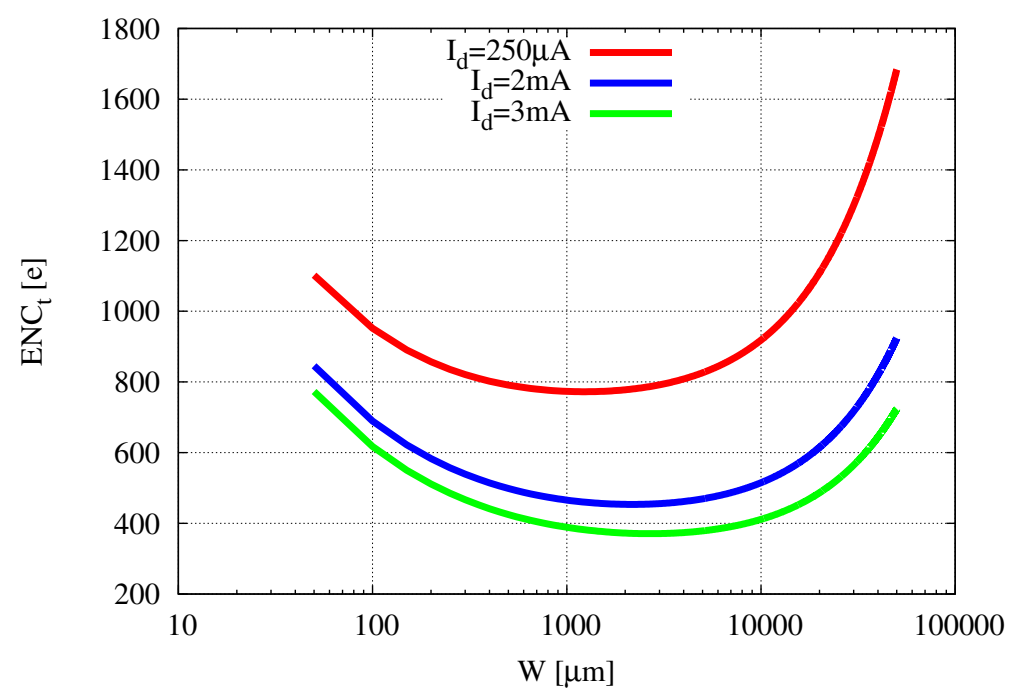

(a)

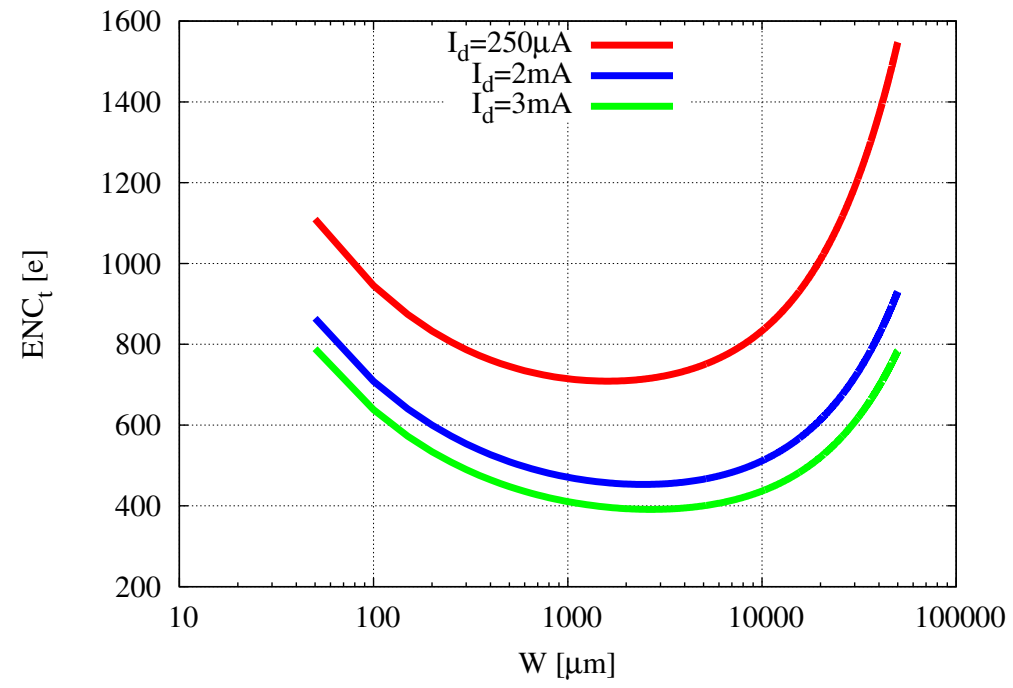

(b)

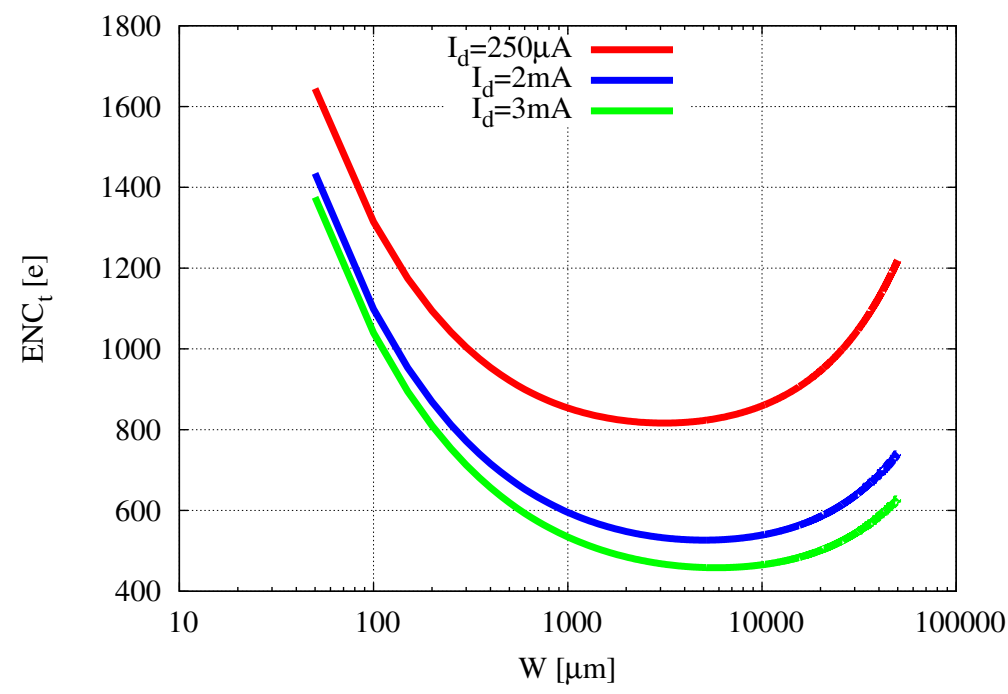

(c)

Figure 3.9: Theoretical $E N C_{t}$ versus $\mathrm{W}$ for $I_{d}=250 u A, I_{d}=2 m A, I_{d}=3 m A$ and (a) $t_{s}=$ $160 \mathrm{~ns}, C_{d}=18.5 \mathrm{pF}$; (b) $t_{s}=80 \mathrm{~ns}, C_{d}=18.5 \mathrm{pF}$; (c) $t_{s}=300 \mathrm{~ns}, C_{d}=40 \mathrm{pF}$. 


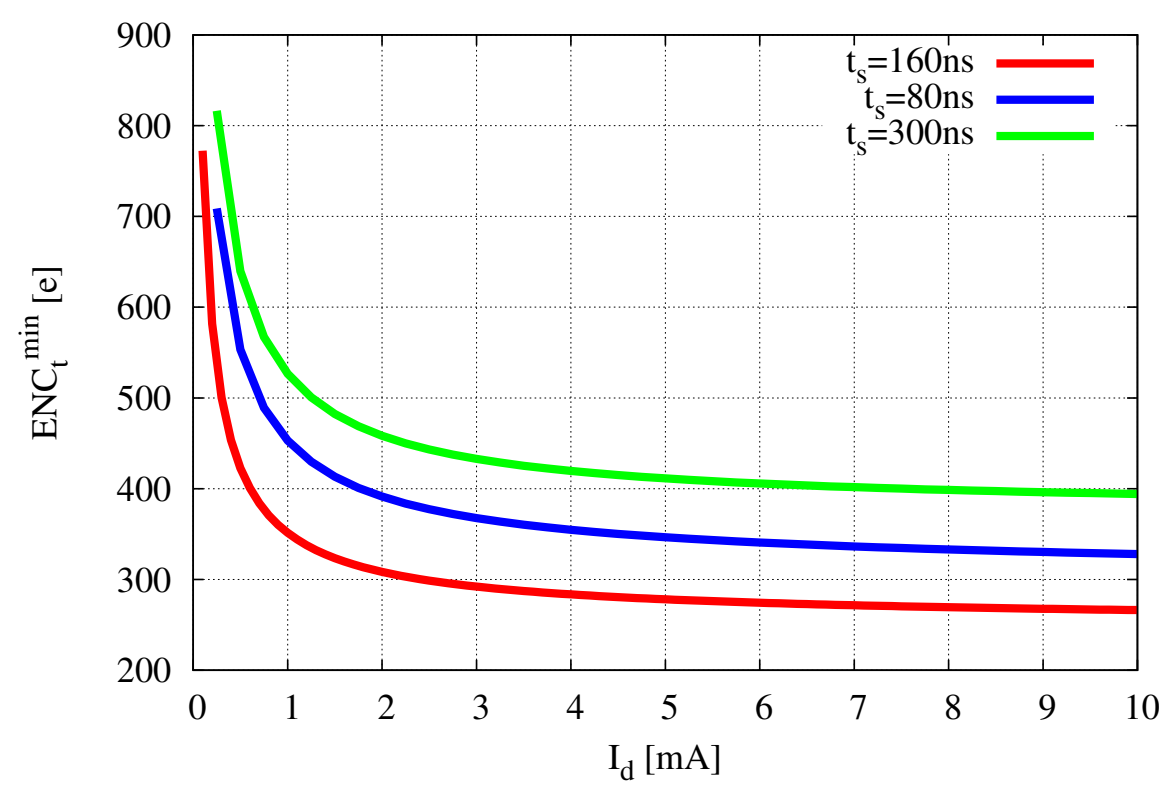

Figure 3.10: Theoretical $E N C_{t}$ versus $I_{d}$ for $t_{s}=160 \mathrm{~ns}, C_{d}=18.5 \mathrm{pF} ; t_{s}=80 \mathrm{~ns}, C_{d}=18.5 \mathrm{pF}$; $t_{s}=300 n s, C_{d}=40 p F$.

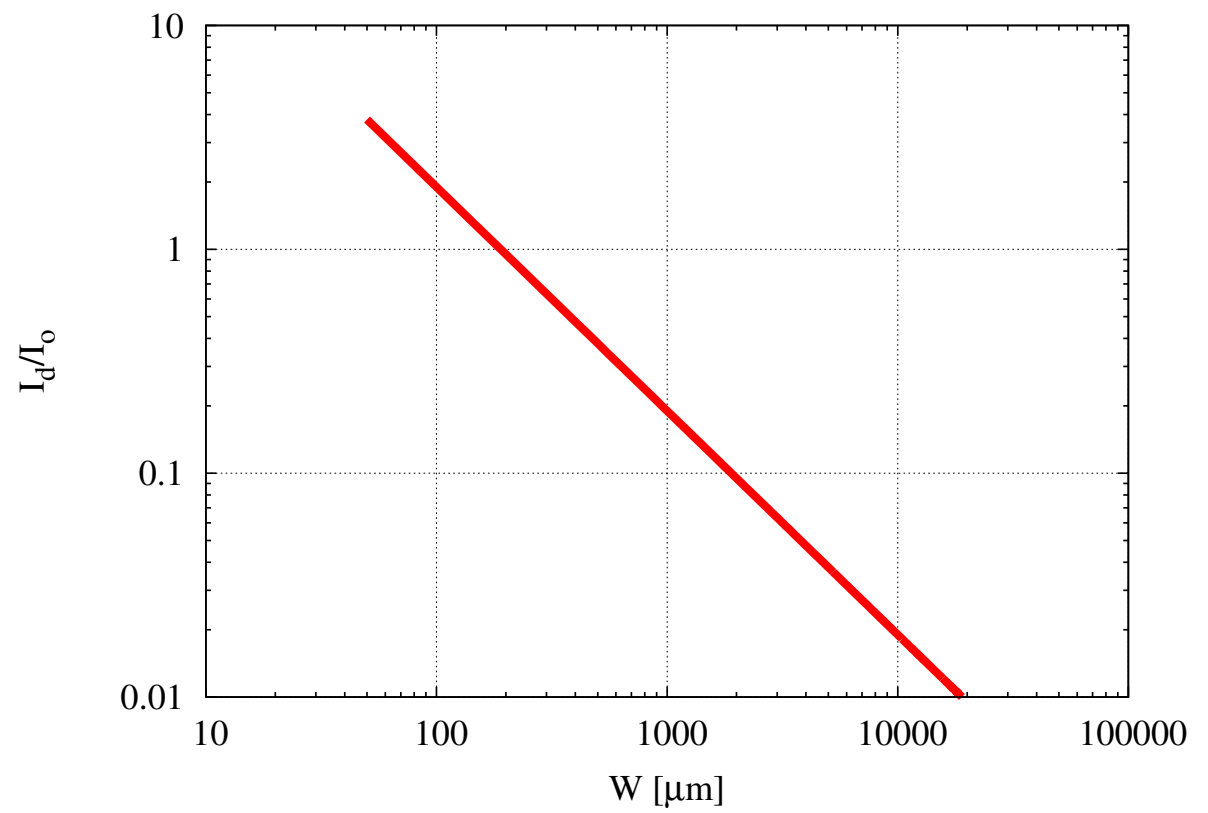

Figure 3.11: Inversion factor $I_{d} / I_{z}$ as a function of $W\left(\mathrm{~L}=L_{m i n}\right)$ and $I_{d}=2 m A$. 
The considerations taken from the ENC performance for the used technology can be utilized to choose $I_{d}$ and $\mathrm{W}$ as a trade-off between $E N C_{t}$ specification of the three peaking time cases and power consumption. The selected values were $\mathrm{W}=2000 \mu \mathrm{m}$ and $I_{d}=2 m A$ which corresponds to $g_{m}=44 \mathrm{mS}$ according to the Tsividis continuous model. Figure 3.11 illustrate the inversion factor $I_{d} / I_{z}$ as a function of $W\left(\mathrm{~L}=L_{\min }\right)$ and $I_{d}=2 m A$. The inversion boundaries in terms of the inversion factor are defined by [ENZ et al., 1995]:

$$
\begin{gathered}
I_{d} / I_{z} \ll: \text { Weak Inversion } \\
I_{d} / I_{z} \approx: \text { Moderate Inversion Inversion } \\
I_{d} / I_{z} \gg: \text { Strong Inversion }
\end{gathered}
$$

Therefore, for the selected dimensions the input transistor operates on the limit between moderate inversion and weak inversion.

\subsubsection{Self-adaptive bias circuit}

The choice of the feedback resistance is a trade-off between low parallel noise (large $R_{f}$ ) and high counting rate (smaller $R_{f}$ ) [GRAMEGNA et al., 1997]. In CMOS technology is very difficult to integrate high-value resistors because it demands high area consumption. The best option to implement high-value resistors with low area is to use a transistor operating in the triode or saturation region. Since the DC current by the feedback resistance is very low (low $V_{d s}$ ), the transistor as resistor will operate in triode, an its resistance is given by:

$$
R_{f}=\frac{1}{\mu C_{o x}(W / L)\left(V_{g s}-V_{t}\right)}
$$

The control sensitivity of this resistor is defined by[GRAMEGNA et al., 1997]:

$$
\left|\frac{d R_{f}}{R_{f}}\right|=\mu C_{O x}(W / L) R_{f}\left|d V_{T O T}\right|
$$

where $d V_{T O T}=d V_{g}+d V_{s}+d V t$ the total variation of the control voltage of the MOS resistor. The factor $\mu C_{o x}$ depend on the process parameters and it can not be controlled to reduce the resistor variations. According to equation (3.43) $L$ must be larger than $\mathrm{W}$ and $\left|d V_{T O T}\right|$ must be low, for low resistor variation. The $\mathrm{W} / \mathrm{L}$ factor can be 


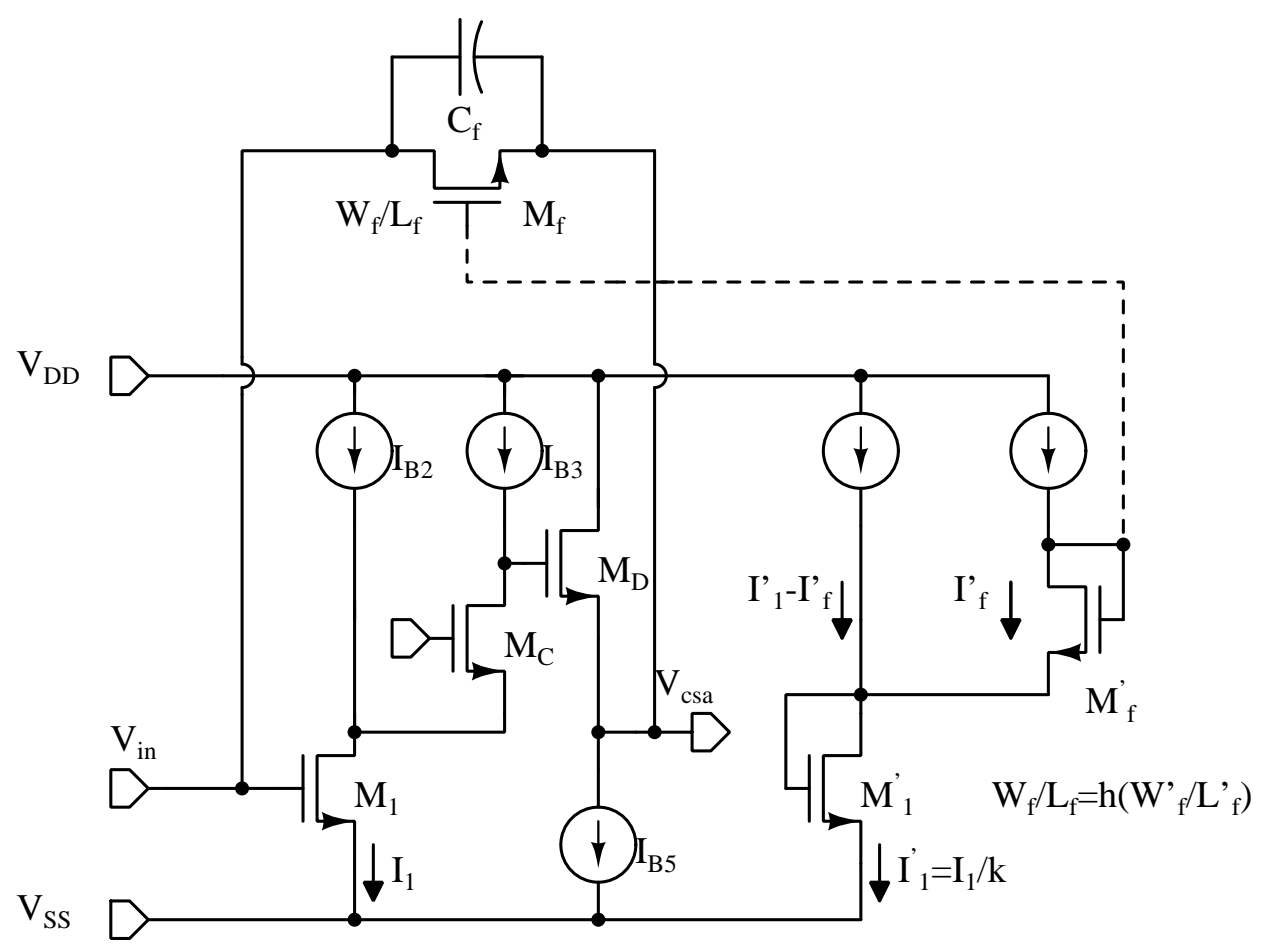

Figure 3.12: Transistor level schematic of the adaptive bias circuit.

defined during the design phase, but $\left|d V_{T O T}\right|$ depend on the supply voltage and the threshold voltage variations. To reduce $\left|d V_{T O T}\right|$ an self-adaptive bias circuit studied in [GRAMEGNA et al., 1997] was implemented. The circuit diagram is shown in Figure 3.12. In this circuit the drain current by $M_{1}^{\prime}$ is a scaled version $\left(I_{1}^{\prime}=I_{1} / k\right)$ of the drain current by $M_{1}$ with channel width of $W_{1}^{\prime}=W_{1} / k\left(L_{1}^{\prime}=L_{1}\right)$, so that the $V_{g s}$ voltage of $M_{1}^{\prime}$ and $M_{1}$ are equal. The $V_{g s}-V_{t}$ voltage of the feedback transistor $M_{f}$ is defined by $M_{f}^{\prime}$ which is a scaled copy of $M_{f}\left(W_{f}^{\prime} / L_{f}^{\prime}=h \cdot W_{f} / L_{f}\right)$. Therefore, $V_{g s}-V_{t}$ of $M_{f}$ is given by:

$$
V_{g s f}-V_{t f}=V_{g s f}^{\prime}-V_{t f}^{\prime}=\sqrt{\frac{2 I_{f}^{\prime}}{\mu C_{o x}\left(W_{f} / L_{f}\right)}}
$$

Substituting equation (3.44) into equation (3.42) we obtained $R_{f}$ of this adaptive circuit:

$$
R_{f}=\sqrt{\frac{h}{2 I_{f}^{\prime} \mu C_{o x}}\left(W_{f}^{\prime} / L_{f}^{\prime}\right)}
$$

In this work $h=48\left(W_{f}^{\prime} / L_{f}^{\prime}\right)=8$ and $I_{f}^{\prime}=500 \mathrm{nA}$ were used to set the requested value of $R_{f}=3 M \Omega$. 


\subsubsection{Schematic of the CSA}

The schematic of the designed CSA is illustrated in Figure 3.13 including the selfadaptive bias circuit studied and the configurable feedback capacitors networks. The switches $S_{1-4}$ adjust the feedback capacitance $C_{f}$ and the differentiator capacitance $C_{d i f f}$ for each peaking time case. They are controlled by 2 bits $(c t s<1: 0>$ ). The voltages generated internally to bias the CSA amplifier will be used in the next first shaper stage. A $C_{d i f f} / C_{f}=R_{f} / R_{p z}$ factor of 8 was chosen where $C_{f}=800 f F$ (value chosen according to the linear range specification). Good matching between $M_{f}-M_{p z}$ and $C_{f}{ }^{-} C_{\text {diff } f}$ for dynamic cancellation of $M_{f} \cdot C_{f}$ pole is required. The transistors that compose the PMOS current mirrors were polarized in strong inversion with $190 \mathrm{mV}$ of saturation voltage to minimize the noise contribution from these transistors. Additionally, decoupling capacitors of $30 \mathrm{pF}$ were used on $V_{B C}$ and $V_{B N}$ to reduce the noise components resulting from $M_{B C}, M_{B 4}$ and $M_{B 5}$. These capacitor was implemented with large gate PMOS/NMOS capacitors since they offer higher capacitance per area than other types of capacitor [LIN, 2011]. The input transistor $M_{1}$ was divided into 40 strips and $M_{2}$ was divided into 12 strips connected at both ends. These values were selected to minimize the poly-gate resistance noise component and to obtain a square layout. The transistors dimensions of the designed CSA are summarized in Table ??. Figure 3.14 shows the layout of the designed CSA in 130nm CMOS technology in which important devices distributions details are presented . 


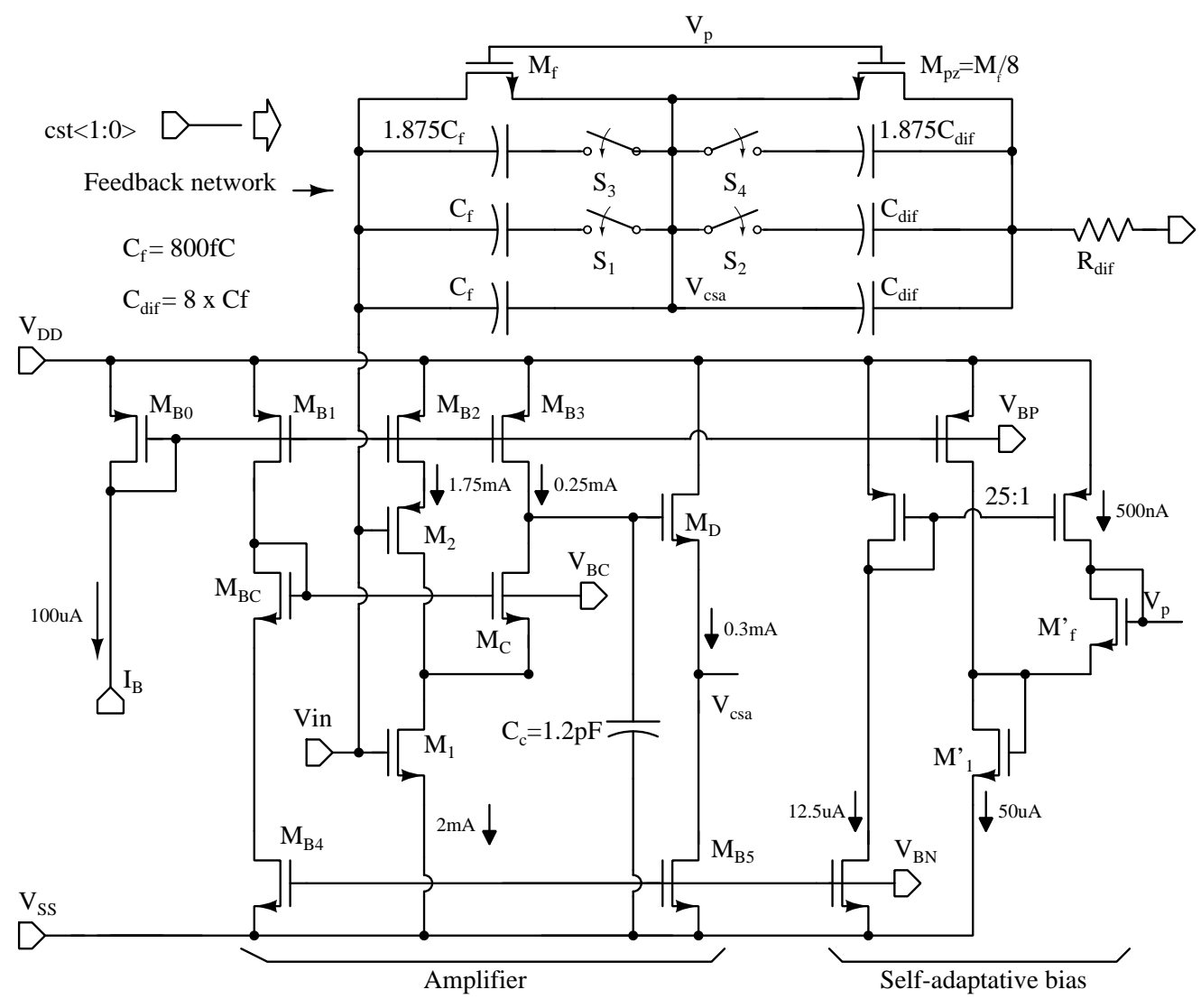

Figure 3.13: Transistor level schematic of the CSA.

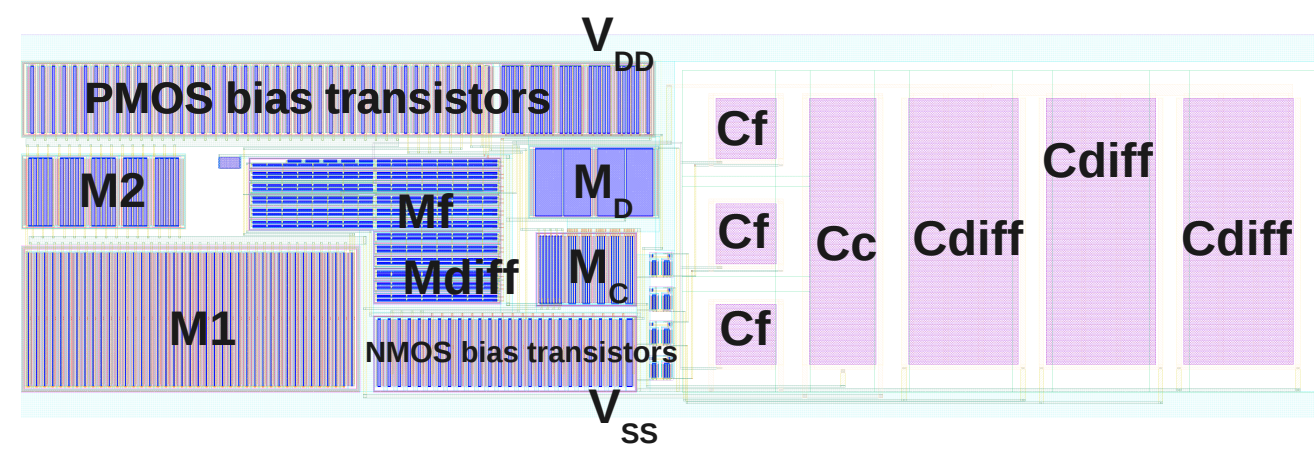

Figure 3.14: Layout of the designed CSA. 


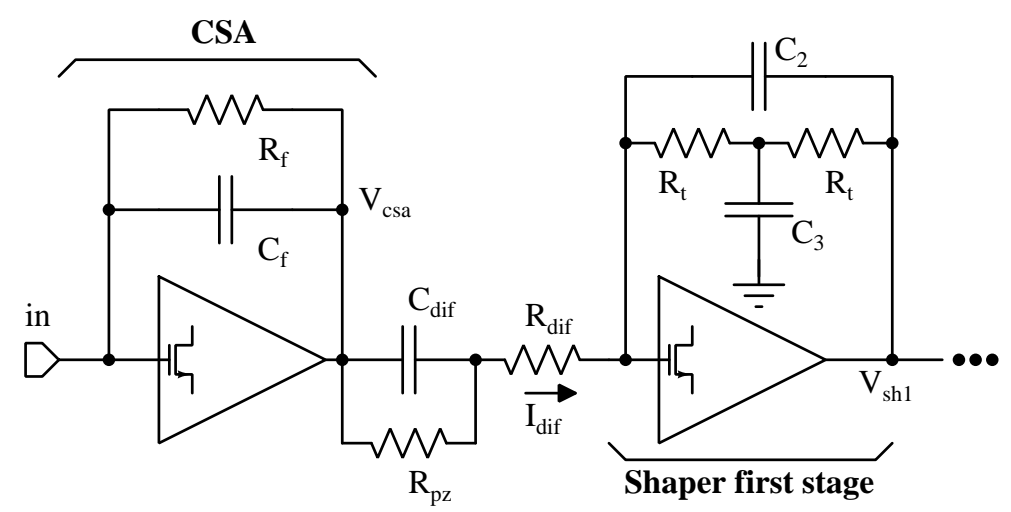

Figure 3.15: Block diagram including the CSA and the shaper first stage.

\subsection{Semi-Gaussian pulse shaper}

The function of the sharper network is to limit the bandwidth of the output signal, in order to avoid aliasing in the subsequent digitization process, and at the same time it has to optimize the overall signal-to-noise ratio. These objectives are achieved by a semi-Gaussian sharper, which is implemented with two low-pass filter stages. Each stage consist of two second-order bridged-T filters connected in cascade. The block diagram including the CSA and the shaper first stage is shown in Figure 3.15. The bridged-T feedback nerwork generates two poles and one zero. The transfer function of this circuit, considering the current by the differentiator resistor $\left(I_{d i f}(s)\right)$ as input and the output voltage of the first shaper stage as output $\left(V_{s h 1}(s)\right)$, is given by:

$$
\frac{V_{s h 1}}{I_{d i f}(s)}=\frac{-2 \cdot R_{T} \cdot\left(1+s R_{T} C_{3} / 2\right)}{s^{2} \cdot R_{T}^{2} C_{2} C_{3}+s \cdot 2 R_{T} C_{2}+1}
$$

where:

$$
\begin{gathered}
\omega_{z}=\frac{2}{R_{f} C_{3}} \\
\omega_{o}=\frac{1}{R_{t} \sqrt{C_{2} C_{3}}} \\
Q_{f}=\frac{1}{2} \sqrt{\frac{C_{3}}{C_{2}}}
\end{gathered}
$$

where $\omega_{z}$ is the zero frequency, $\omega_{o}$ is the cut-off filter frequency and $Q_{f}$ is the quality factor. The transfer function considering the current by the differentiator resistor $\left(I_{d i f}(s)\right)$ as output and the output voltage of the CSA amplifier as input $\left(V_{c s a}(s)\right)$, is gien 
by:

$$
\frac{I_{d i f}(s)}{V_{c s a}(s)}=\frac{1}{R_{p z}+R_{1}} \cdot \frac{1+s R_{p z} C_{d i f}}{1+s\left(R_{p z} \| R_{d i f}\right) C_{d i f}}
$$

where:

$$
\begin{gathered}
\omega_{p z}=\frac{1}{R_{p z} C_{d i f f}} \\
\omega_{r}=\frac{1}{\left(R_{p z} \| R_{d i f}\right) C_{d i f}}
\end{gathered}
$$

where $\omega_{p z}$ is the zero frequency of the pole-zero cancellation and $\omega_{r}$ is a remanent pole. In order to obtain the Semi-Gaussian transfer function defined by the equation (2.8), the zero $\left(\omega_{z}\right)$ should cancel the remanent pole of the last stage. The transistor level schematic of the first stage of the shaper is shown in Figure 3.16a. It is a scaled-down version as the CSA. In order to provide the second shaper with a differential mode input, a copy of the first shaper connected in unity gain configuration is included. A low DC gain (closed-loop) of about 2V/V for the first stage of the pulse shaper was chosen to avoid the saturation of the output buffer. The $V_{g s}$ voltage of the input transistor of the CSA and first stage of the shaper should be equals in favor to avoid DC current by $R_{d i f}$. A DC current by $R_{\text {dif }}$ will generate a ofset level that will be reflected on the pulse shaper output. The second shaper (Figure 3.16b) consists of a fully differential amplifier with a Miller configuration and a common mode feedback (CMFB) network. It has the same functionality as the first shaper and implements two other poles and a zero and creates the $\mathrm{CR}-(\mathrm{RC})^{4}$ semi-Gaussian shaper together with the differentiator and the first shaper stage. The CMFB network establishes a stable common-mode voltage $V_{C M}$ of $600 \mathrm{mV}$ at the output of the second shaper. The chosen CMFB network (Figure 3.16b) consists of a resistor-capacitor network. This configuration takes the average of the two outputs, $V_{s h 2 p}$ and $V_{s h 2 n}$, and compares it with an externally given voltage $V_{C M}$ and adjust the polarization current of the first stage of the amplifier. The sensitivity of the front-end is controlled by the $R_{g}$ array of parallel resistances (Figure $3.16 \mathrm{~b})$ which are switched by 3 control bits $(\mathrm{cg}<2: 0>)$. Additionally, the peaking time of the Semi-Gaussian shaper is adjusted for each experiment case (TPC:160ns, $80 \mathrm{~ns}$ and $\mathrm{MCH}: 300 \mathrm{~ns}$ ) by 2 bits (cts $<1: 0>$ ) which control an array of parallel capacitors. These front-end configurations are performed with transmission gates used as low resistance switches. The bias current for each stage of the front-end is generated by a basic 
constant-transconductance bias circuit presented [JOHNS; MARTIN, 2008] in Figure 3.17a. In order to match the $V_{g s}$ of the input transistor of the CSA and the first stage of the shaper, them have common bias voltages $\left(V_{B N}, V_{B P}\right.$ and $V_{B C}$ in Figure 3.16a). The resistance used to generated the bias bias current is external, and the circuit was design to generate a typical current of $12.5 \mu \mathrm{A}\left(R_{E X T}=7.2 \mathrm{~K} \Omega\right)$ which is replicated by current mirrors to bias the CSA, the pulse shaper and the Non-inverting stage of each one of the 32 channels.

The non-inverting stage is composed by two miller opamp where one was implemented with NMOS differential pair for input signals above $V_{C M}$, and the other with PMOS differential pair for signal below $V_{C M}$ (Figure 3.17b). A class AB output stage was used for both opamp to have capability of driving the ADC input capacitance. The Non-inverting stage requires of $600 \mathrm{mV}, 450 \mathrm{mV}$ and $750 \mathrm{mV}$ voltages reference to generate the baseline differential output voltage. The baseline voltage is defined as the differential output voltage when a specific channel is not pulsing. The typical value of baseline of the designed front-end is $-1 \mathrm{~V}\left(V_{\text {outp }}=100 \mathrm{mV} V_{\text {outn }}=1.1 \mathrm{~V}\right)$. 


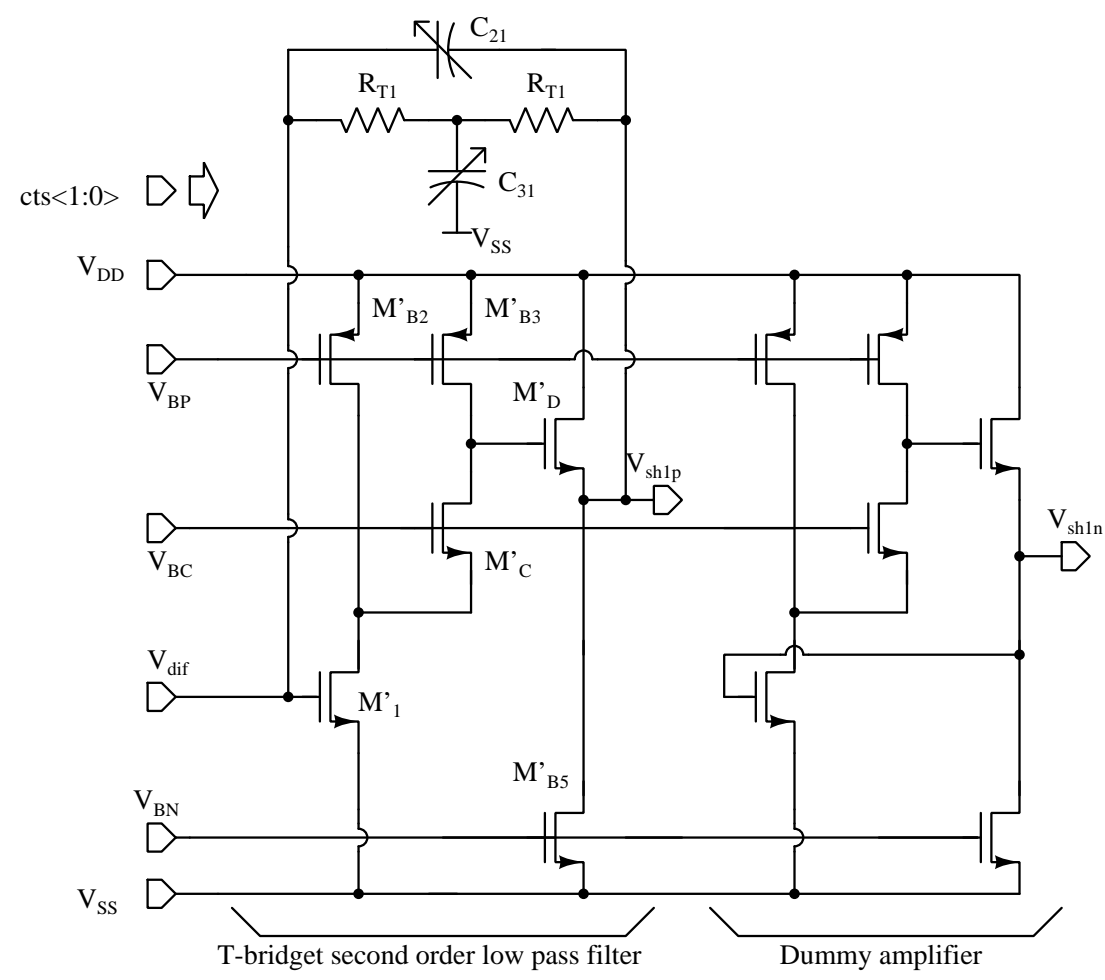

(a)

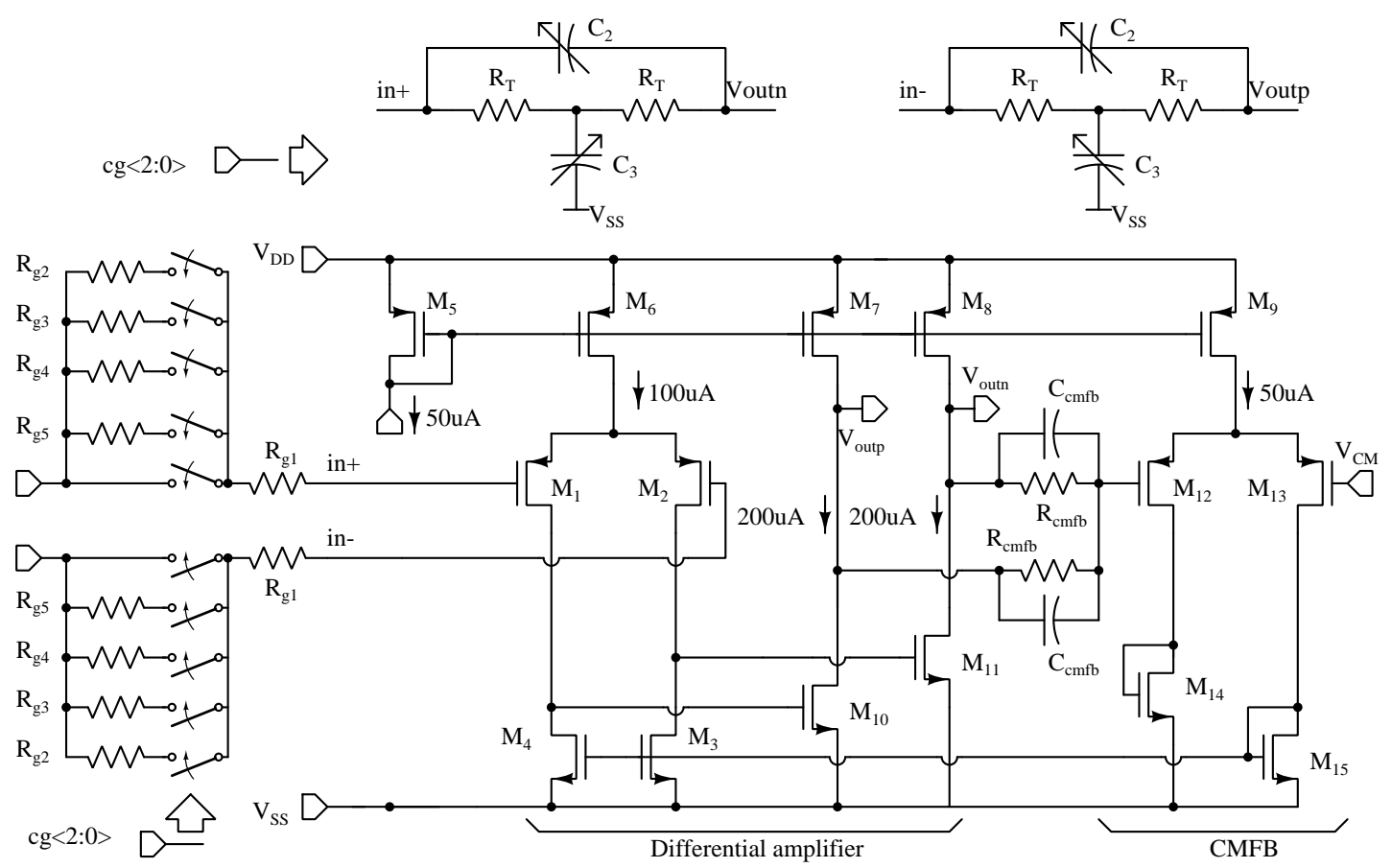

(b)

Figure 3.16: Transistor level schematic of: (a)Pulse shaper first stage; (b) Pulse shaper second stage 


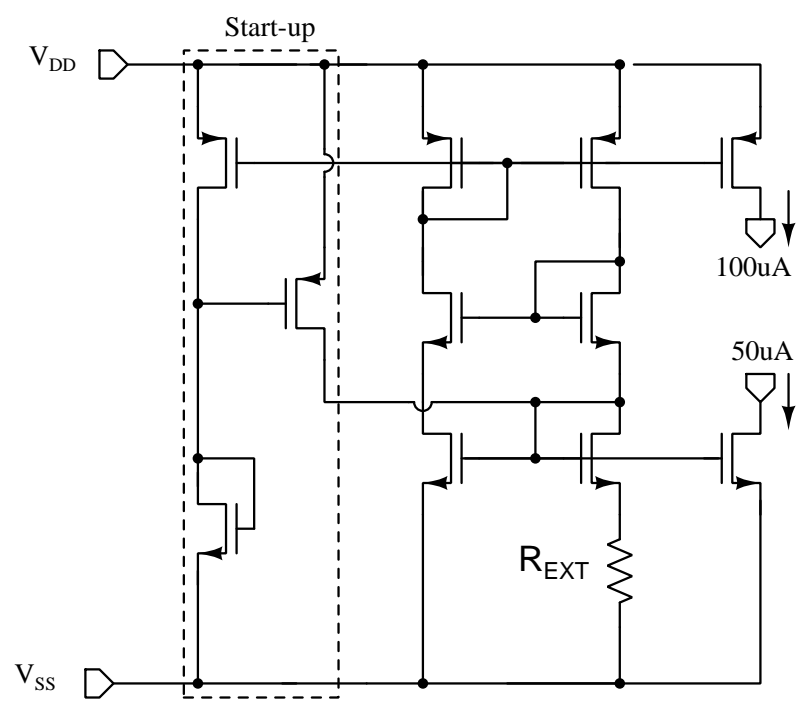

(a)

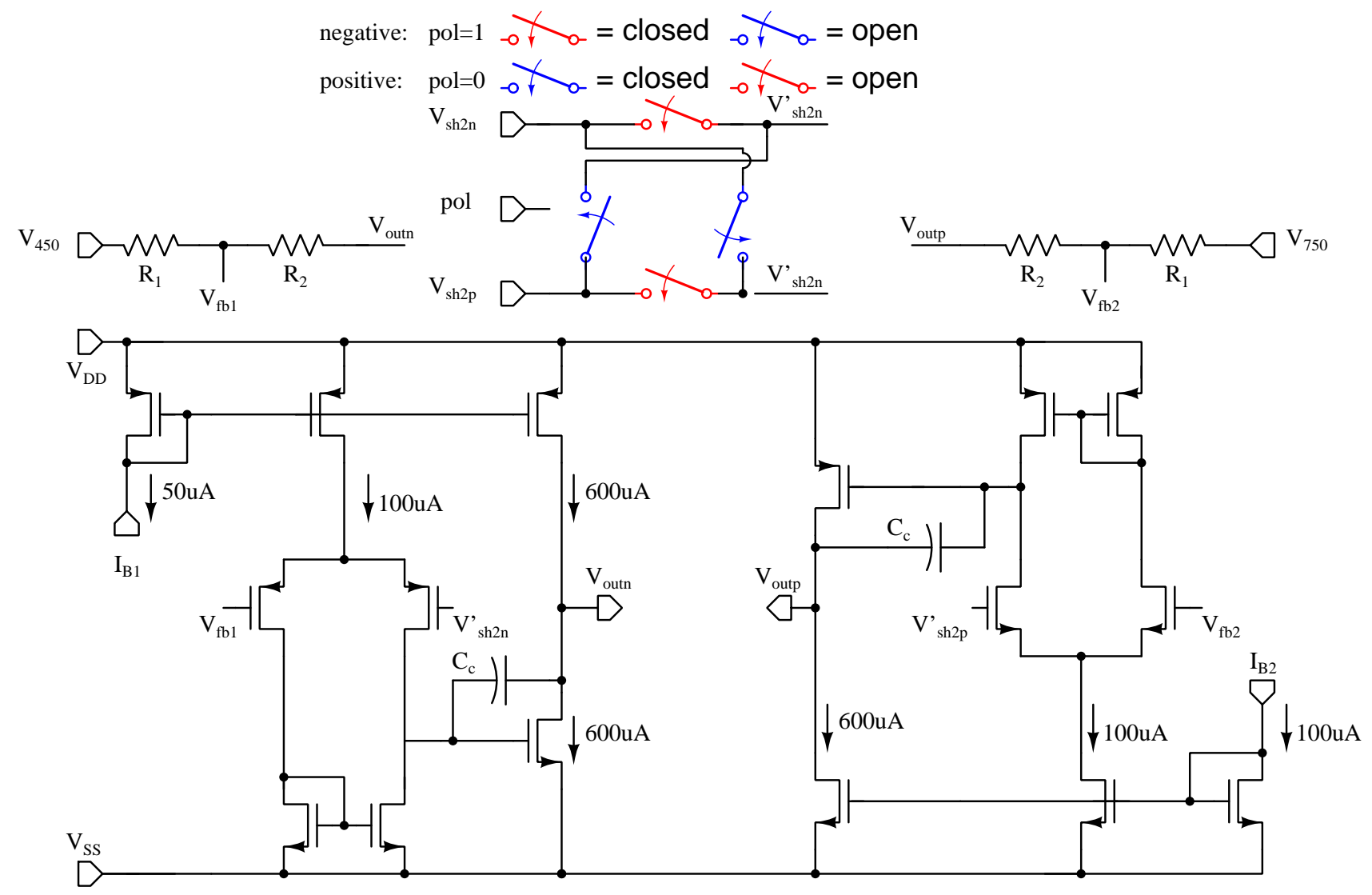

(b)

Figure 3.17: Transistor level schematic of: (a) Bias current generator; (b) Non-inverting stage 


\section{Chapter 4}

\section{Noise and PSRR improvement}

\section{technique}

In order to obtain an ASIC integrating 32 channels per chip, the design of the proposed front-end requires low area, low power consumption, but at the same time requires low noise. Whith this objective, a new Noise and PSRR (Power Supply Rejection Ratio) improvement technique for the CSA design without power and area impact is presented in this chapter. The analysis and equations of the proposed circuit are discussed which were verified by electrical simulations and experimental tests of a produced chip with 5 channels of the designed front-end. The experimental results are presented in the next chapter.

As discussed in section 3.2.5, the noise components generated by the PMOS bias current mirror have an important impact on the total noise specification specially for low voltage high dynamic range front-ends design. A method of reducing these noise components is the introduction of a gate-source decoupling capacitance $\left(C_{B P}\right.$ in Figure 3.1) on the current mirror transistors $\left(M_{B 0}\right)$ which will perform a Low Pass Filter (LPF) function in combination with the $M_{B 0}$ transistor transconductance. The dominant pole of this LPF combination is given by $g_{m B 0} / C_{B P}$ which must be placed at frequencies lower than the frequency band of the Semi-Gaussian Pulse Shaper.

The $\mathrm{ENC}_{M B 0}$ component in terms of the bias decoupling capacitance $C_{B P}$ and the $\left(B_{02}+B_{03}\right)$ factor (by definition $B_{02}=\frac{I_{1}}{I_{B}}$ and $B_{03}=\frac{I_{2}}{I_{B}}$ in Figure 3.1) of the original NMOS input transistor topology with $C_{d}=18.5 p F, t_{s}=160 \mathrm{~ns}$, Sensitivity $($ Sen $)=30 \mathrm{mV} / \mathrm{fC}$ and $R_{S}=0 \Omega$ is shown in Figure 4.6. This graph display that $300 \mathrm{pF}$ of bias decoupling 


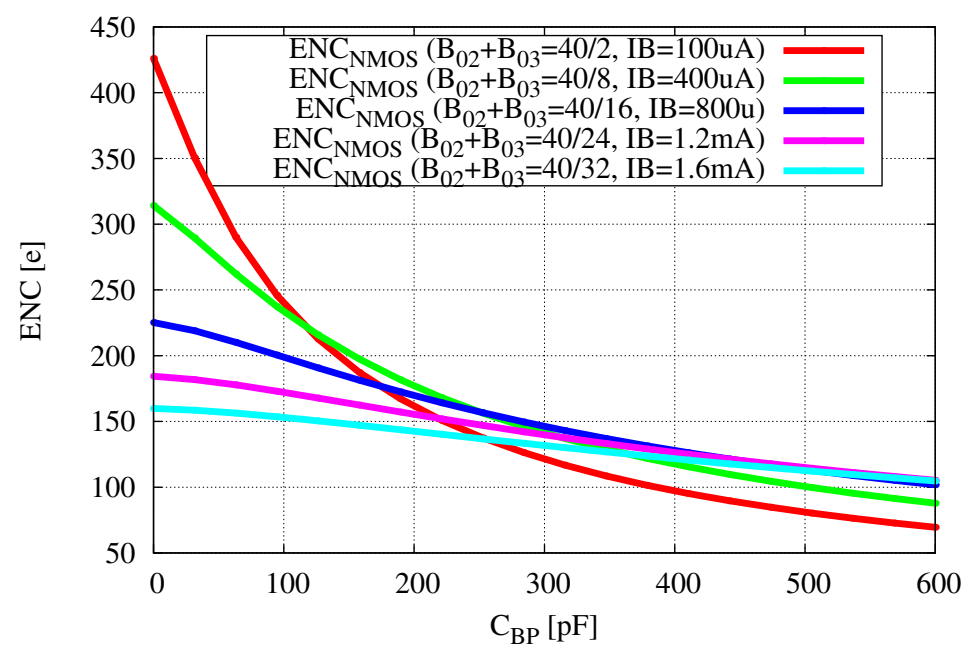

Figure 4.1: $\mathrm{ENC}_{M B 0}$ component in terms of the bias decoupling capacitance $\left(C_{B P}\right)$ simulation of the original NMOS input transistor topology with $C_{d}=18.5 p F, t_{s}=160 \mathrm{~ns}, S e n=30 \mathrm{mV} / \mathrm{fC}$ and $R_{s}=0 \Omega$.

capacitance is necessary with the NMOS input transistor topology to achieve a significant $\mathrm{ENC}_{M B 0}$ reduction. Similarly, a bias current $\left(I_{B}\right)$ higher than $1.2 \mathrm{~mA}\left(B_{02}+B_{03}\right.$ $=40 / 32)$ when $C_{B P}=0 \mathrm{pF}$ is necessary with the NMOS input transistor topology to achieve a significant $\mathrm{ENC}_{M B 0}$ reduction. This simulation result shows that the decoupling capacitance value can be reduced for small current copy factors $\left(B_{02}\right.$ and $B_{03}$ ) but this would lead to an excessive increase of the power consumption. Another way to reduce the decoupling capacitance area is to use MOS capacitors. In modern CMOS technologies MOS capacitors have higher capacitance per $\mu m^{2}$ than MIM (Metal-Insulator-Metal) capacitor or MOM (metal-oxide metal). Nevertheless, MOS gate capacitance is very sensitive to ionizing radiation, specially when large gate areas are used [SRINIVASAN; PANDYA, 2011].

In an effort to minimize the bias noise components and to improve the CSA PSRR, this work proposes two simple techniques which alters the original topology (Figure 3.1) and are analyzed in the next section.

The low PSRR at at the frequency band of the Semi-Gaussian shaper of the CSA is other limitation for low noise circuit operation. Any noise component from the power supply will be amplified by the pulse shaper to the front-end output if the CSA PSRR does not attenuate it. Furthermore, important crosstalk components will be generated if the transient voltage drops of the supply voltage are not compensated by the CSA. In this sections a PSRR analysis of the CSA with the original NMOS input transistor topology and the PSRR improvement obtained with the proposed input transistor will 


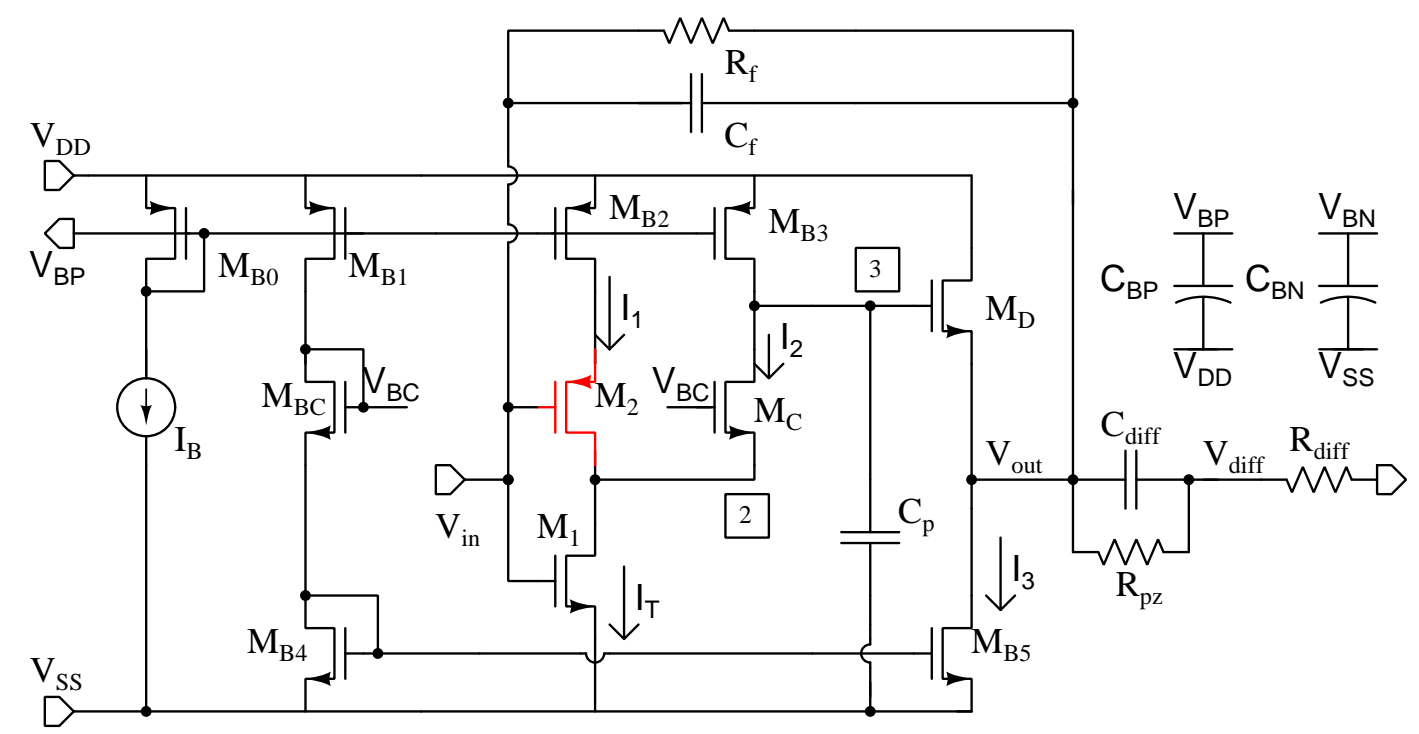

Figure 4.2: Schematic of the CSA with the proposed PMOs input transistor

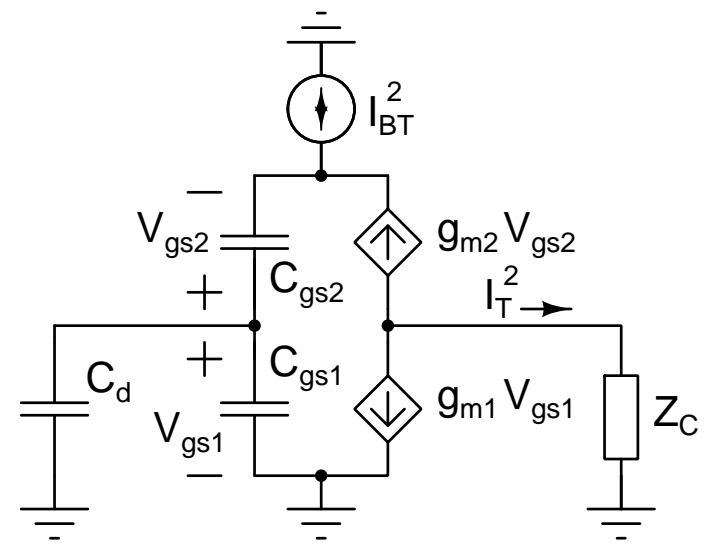

Figure 4.3: Small signal equivalent circuit of the proposed topology adding a PMOS input transistor $\left(M_{2}\right)$.

be presented.

\subsection{PMOS-NMOS input transistor topology}

The first technique proposes adding a PMOS input transistor $\left(M_{2}\right)$ as showed in Figure 4.2 called PMOS-NMOS input transistor topology from this point. The new $M_{2}$ transistor has not influence on the total amplifier transconductance because $M_{B 2}$ output resistance is degenerating the $M_{2}$ transconductance. The small signal circuit of the proposed topology is presented in Figure 4.3. The gate-drain capacitance of $M_{1}$ and $M_{2}$ have not been considered and $Z_{C}$ represents the parallel of the $M_{1}$ and $M_{2}$ output resistance and the input impedance seen on the $M_{C}$ transistor source. $I_{B T}^{2}$ represents the total noise current resulting from the bias network assuming that the ratio between $M_{B 0}$ and $M_{B 2}$ much greater than the ratio between $M_{B 0}$ and $M_{B 3}$. The $I_{B T}^{2}$ noise 
current referred to the amplifier input $\left(V_{i B T}^{2}\right)$ can be determined as follows:

$$
\begin{gathered}
I_{T}^{2}=-\left(g_{m 1}^{2} V_{g s 1}^{2}+g_{m 2}^{2} V_{g s 2}^{2}\right) \\
I_{B T}^{2}=-\left(g_{m 2}^{2}+s^{2} C_{g s 2}^{2}\right) V_{g s 2}^{2} \\
V_{g s 1}^{2}=-\left[\frac{1}{s^{2}\left(C_{g s 1}+C_{d}\right)^{2}}\right] s^{2} C_{g s 2}^{2} V_{g s 2}^{2} \\
V_{g s 1}^{2}=-\left(\frac{C_{g s 2}^{2}}{\left(C_{g s 1}+C_{d}\right)^{2}}\right) V_{g s 2}^{2}
\end{gathered}
$$

From (4.1) and (4.4)

$$
I_{T}^{2}=-\left(-\frac{g_{m 1}^{2} C_{g s 2}^{2}}{C_{g s 1}^{2}+C_{d}^{2}}+g_{m 2}^{2}\right) V_{g s 2}^{2}
$$

From (4.2) and (4.5)

$$
\begin{gathered}
I_{T}^{2}=I_{B T}^{2} \frac{\left(g_{m 2}^{2}-\frac{\left.g_{m 1}^{2} C_{g s 2}^{2}\right)}{\left.C_{g s 1}^{2}+C_{d}^{2}\right)}\right.}{g_{m 2}^{2}+s^{2} C_{g s 2}^{2}}=I_{B T}^{2} \frac{\left[1-\frac{g_{m 1}^{2} C_{g s 2}^{2}}{g_{m 2}^{2}\left(C_{g s 1}+C_{d}\right)^{2}}\right]}{1+s^{2} \frac{C_{g s 2}^{2}}{g_{m 2}^{2}}} \\
V_{i B T}^{2}=\frac{I_{B T}^{2}}{g_{m 1}^{2}} \frac{\left[1-\frac{g_{m 1}^{2} C_{g s 2}^{2}}{g_{m 2}^{2}\left(C_{g s 1}+C_{d}\right)^{2}}\right]}{1+s^{2} \frac{C_{g s 2}^{2}}{g_{m 2}^{2}}}
\end{gathered}
$$

From equation (4.7) $V_{i B T}^{2}$ can be canceled when:

$$
\frac{g_{m 1}}{g_{m 2}}=\frac{C_{d}+C_{g s 1}}{C_{g s}}
$$

Considering $C_{g s 1}^{2} \ll C_{d}^{2}$ then $C_{g s}^{2}+C_{d}^{2} \approx C_{d}^{2}$. Therefore, the zero noise condition is defined by:

$$
\frac{g_{m 1}}{g_{m 2}} \approx \frac{C_{d}}{C_{g s}}
$$

The above equation determines the relation between $M_{1}$ and $M_{2}$ transconductance to cancel the bias noise for a specific detector capacitance value. In addition to the bias 
noise cancellation effect of the $M_{2}$ transistor, it introduces an extra ENC component (channel thermal noise and flicker noise) which can be determined by:

$$
E N C_{2}^{2}=\left[\frac{D_{c h p} C_{t}^{2}}{g_{m 2} \tau_{s}}+\frac{D_{f p} C_{t}^{2}}{(W L)_{2}}\right] \frac{1}{g_{m 1}^{2}}
$$

The dimensions $(W L)_{2}$ and transconductance $\left(g_{m 2}\right)$ of $M_{2}$ should be chosen so that its contribution be lower than the canceled noise component. Figure 4.4 shows the ENC component of $M_{B 0}, M_{B 2}$ and $M_{B 3}$ in relation to the detector capacitance of the original NMOS input transistor topology (Figure $4.4 \mathrm{a}$ ) and the proposed PMOS-NMOS input transistor topology (Figure 4.4 (b)) for $C_{B P}=0 \mathrm{pF}, t_{s}=160 \mathrm{~ns}, S e n=30 \mathrm{mV} / \mathrm{fC}$ and $R_{S}=0 \Omega$. From these results it is clear the cancellation effect generated by the $M_{2}$ transistor on the $\mathrm{ENC}_{M B 0}$ and $\mathrm{ENC}_{M B 2}$ components around $C_{d}=18.5 \mathrm{pF}$.

The CSA was designed for $g_{m 1}=44 \mathrm{mS}, g_{m 2}=10 \mathrm{mS}, C_{d}=18.5 \mathrm{pF}$ (typical detector capacitance value for the TPC case), $C_{g s 2}=4.2 \mathrm{pF}, I_{B}=100 \mu A, B_{02}=35 / 2$ and $B_{03}=5 / 2$. According to the equation 4.9 and the selected design parameters, the detector capacitance for zero bias noise condition is $18.41 \mathrm{pF}$ which is close to the schematic simulation results presented in Figure 4.4. The zero bias noise condition was adjusted to the TPC typical detector capacitance case $(18.5 \mathrm{pF})$ because it is the more critical noise specifications of front-end design.

Figure 4.5 illustrates the total ENC obtained with the original NMOS input transistor topology $\left(E N C_{N M O S}\right)$ for $C_{B P}=0 p F$ and $C_{B P}=300 p F$, and with the proposed PMOS-NMOS input transistor topology $\left(E N C_{P N M O S}\right)$ in term of the detector capacitance for $C_{B P}=0 \mathrm{pF}, t_{S}=160 \mathrm{~ns}, S e n=30 \mathrm{mV} / \mathrm{fC}$ and $R_{S}=0 \Omega$. A reduction of around $420 \mathrm{e}$ is achieved with the PMOS-NMOS input transistor topology for $C_{d}=18.5 \mathrm{pF}$ and $C_{B P}=0 p F$, also a reduction of around $420 \mathrm{e}$ is obtained with the PMOS-NMOS input transistor topology for $C_{d}=18.5 \mathrm{pF}$ and $C_{B P}=0 p F$. The NMOS input transistor topology presents lower noise for detector capacitance values bellow of 12pF, but PMOS-NMOS input transistor topology achieves a better noise performance for detector capacitance values above $12 \mathrm{pF}$, which is the minimum detector capacitance specification for the ALICE TPC.

A important result obtained with the PMOS-NMOS input transistor topology is a lower variation than the total ENC specification in relation to the detector capacitance 


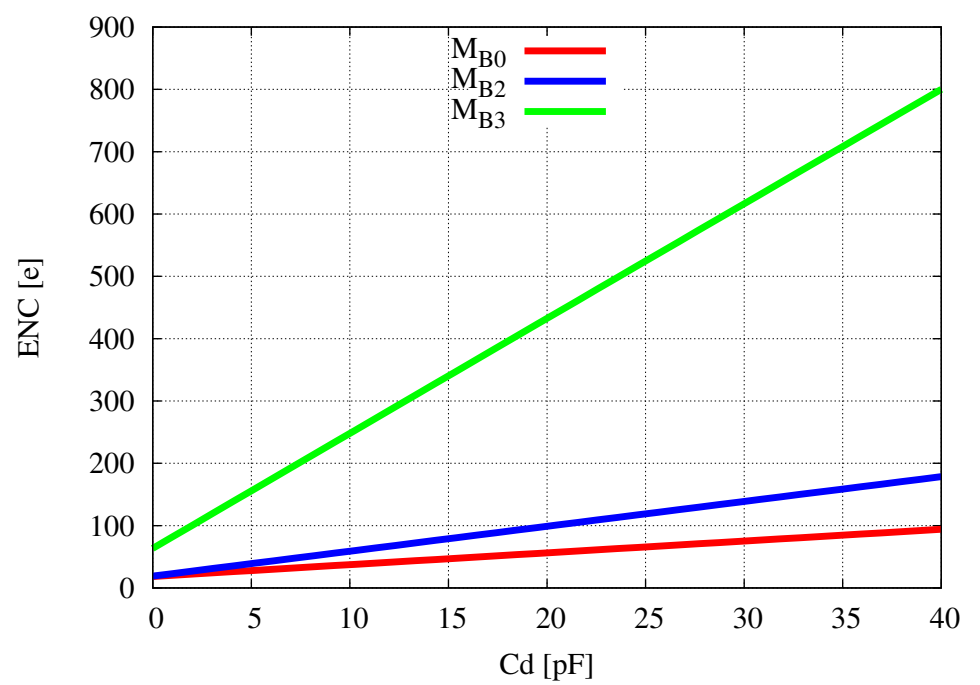

(a)

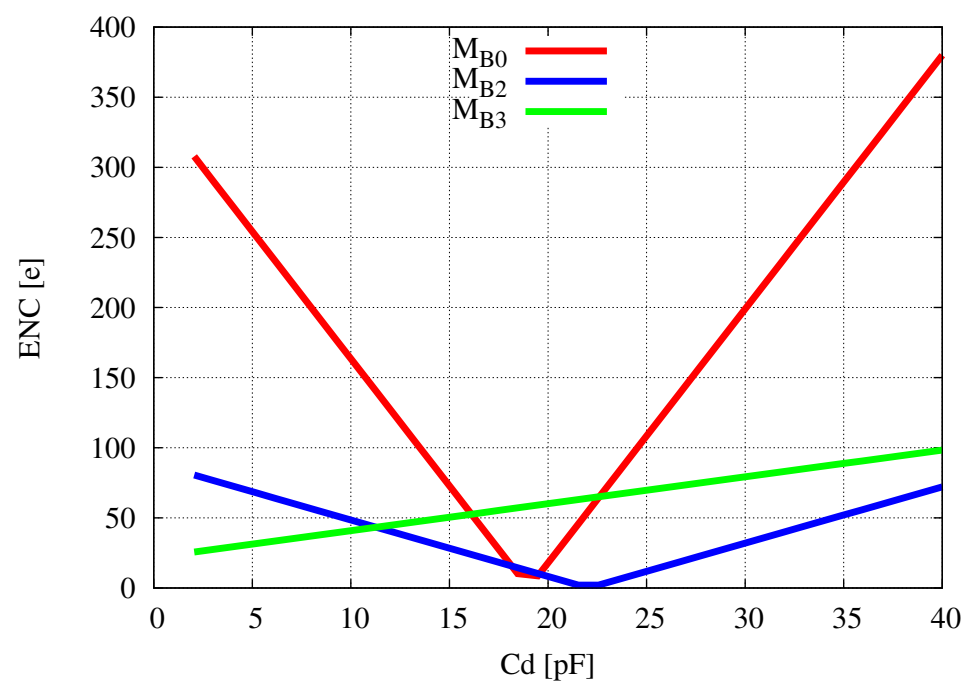

(b)

Figure 4.4: ENC components of $M_{B 0}, M_{B 2}$ and $M_{B 3}$ in relation to the detector capacitance for $t_{s}=160 \mathrm{~ns}, S e n=30 \mathrm{mV} / \mathrm{fC}$ and $R_{s}=0 \Omega$ : (a) NMOS input transistor topology (b)PMOS-NMOS input transistor topology

(around 5\% according to Figure 4.4 b) if compared with NMOS input transistor topology around (40\% for $C_{B P}=0 p F$ and $15 \%$ for $C_{B P}=300 p F$ according to Figure $\left.4.4 \mathrm{a}\right)$. The detector capacitance which is defined by the detector pad capacitance and the interconnection capacitance can vary from channel to channel of the gaseous detector. Low ENC increases when $C_{d}$ increase is desired because the detector pad size can be increased without losing noise performance.

The total ENC in terms of the bias decoupling capacitance $\left(C_{B P}\right)$ and the $B_{02}+B_{03}$ factor with the original NMOS input transistor topology and with the proposed PMOSNMOS input transistor topology for $C_{d}=18.5 p F, t_{s}=160 \mathrm{~ns}, S e n=30 \mathrm{mV} / \mathrm{fC}$ and $R_{S}=0 \Omega$ is shown in Figure 4.6. This graph display that $560 \mathrm{pF}$ of bias decoupling capacitance 


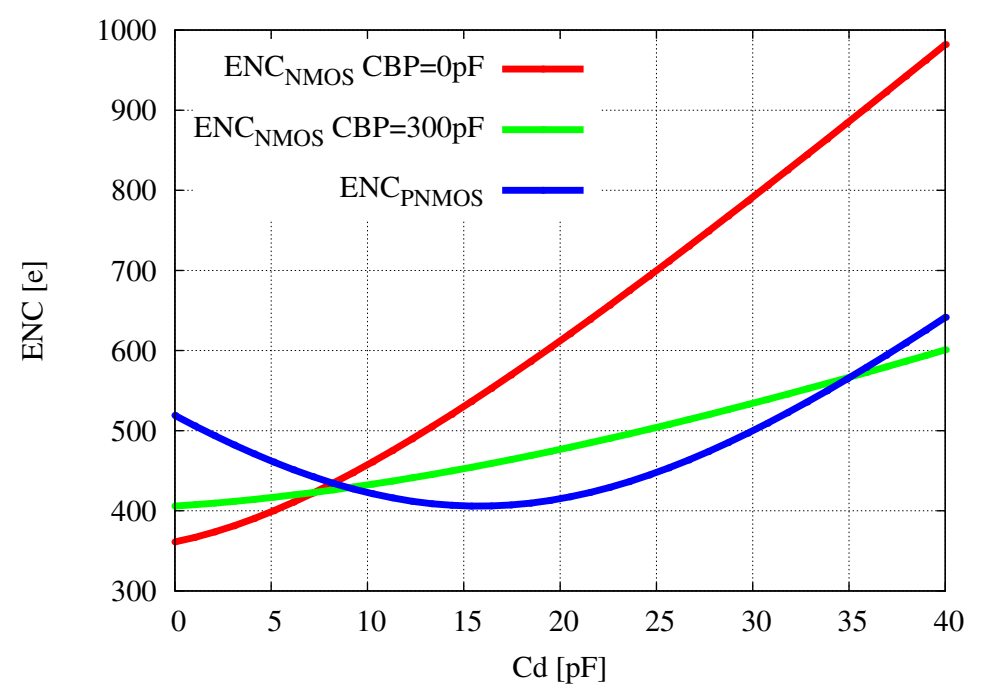

Figure 4.5: Comparison between the total ENC obtained with the original NMOS input transistor topology $\left(E N C_{N M O S}\right)$ and with the proposed PMOS-NMOS input transistor topology $\left(E N C_{P N M O S}\right)$ in term of the detector capacitance for $C_{B P}=0 \mathrm{pF}, t_{s}=160 \mathrm{~ns}$, $S e n=30 \mathrm{mV} / \mathrm{fC}$ and $R_{s}=0 \Omega$.

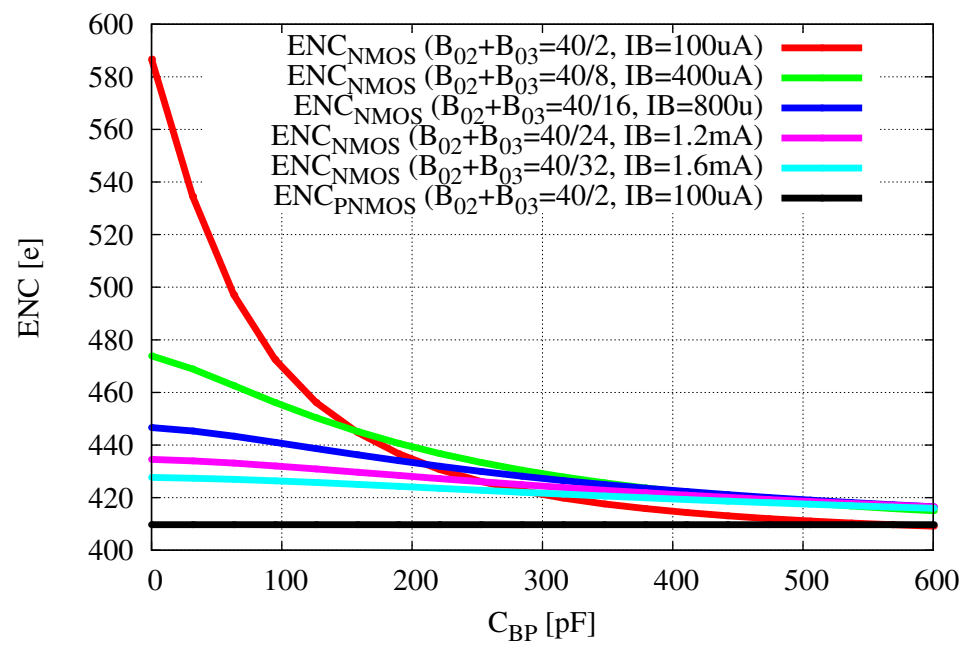

Figure 4.6: Total ENC in terms of the bias decoupling capacitance $\left(C_{B P}\right)$ of the original NMOS input transistor topology and with the proposed PMOS-NMOS input transistor topology $\left(E N C_{P N M O S}\right)$ for $C_{d}=18.5 p F, t_{s}=160 \mathrm{~ns}, S e n=30 \mathrm{mV} / \mathrm{fC}$ and $R_{s}=0 \Omega$.

is necessary in the NMOS input transistor topology when $B_{02}+B_{03}=40 / 2$ to achieve a similar ENC performance to the PMOS-NMOS input transistor topology. Likewise, a bias current $\left(I_{B}\right)$ of $1.6 \mathrm{~mA}\left(B_{02}+B_{03}=40 / 32\right)$ when $C_{B P}=0 \mathrm{pF}$ is necessary with the NMOS input transistor topology to have a ENC performance close to the PMOS-NMOS input transistor configuration.

In addition to the noise cancellation effect on the $M_{B 0}$ and $M_{B 2}$, the proposed topology also cancels the noise components from bias current circuit $\left(I_{B}\right)$ because the analysis presented in this section includes all bias noise components referred to the $M_{2}$ source. A simulation of the total ENC generated by the designed bias current 


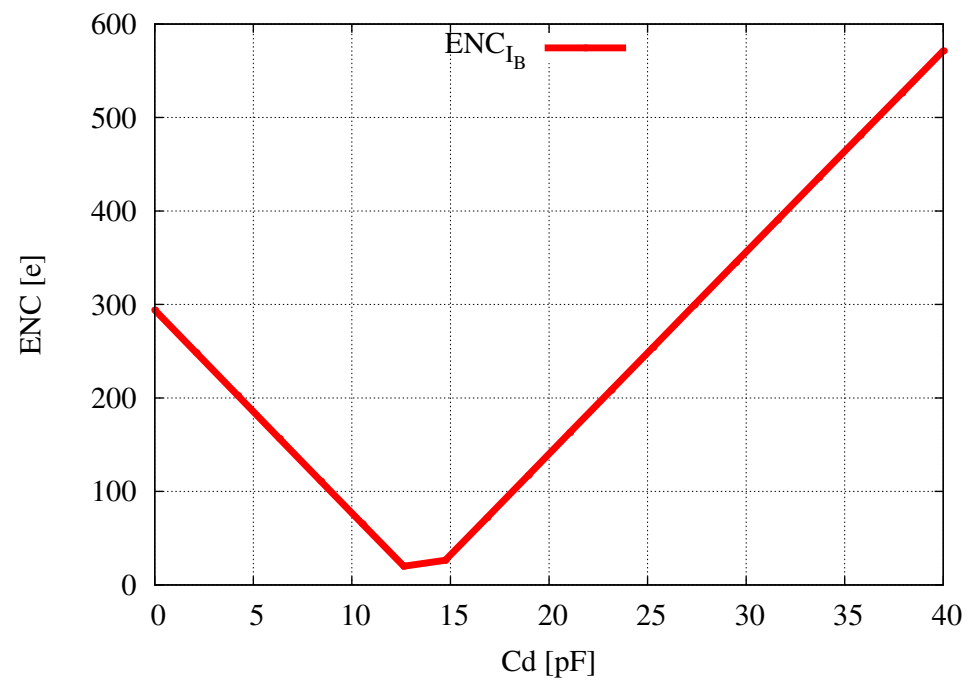

Figure 4.7: Total ENC generated by the designed bias current for $C_{d}=18.5 p F, t_{s}=160 \mathrm{~ns}$, Sen $=30 \mathrm{mV} / \mathrm{fC}$ and $R_{s}=0 \Omega$.

$\left(\mathrm{ENC}_{I_{B}}\right)$ circuit was performed for $C_{d}=18.5 \mathrm{pF}, t_{s}=160 \mathrm{~ns}$, Sen $=30 \mathrm{mV} / \mathrm{fC}$ and $R_{s}=$ $0 \Omega$, and it is shown in Figure 4.7. Decoupling capacitors on the bias current circuit were not included in this simulation. This results proves that the theoretical analysis realized in this section is correct, and the that proposed topology helps to relax the noise specification of the the bias current circuit design.

It might be imagined that the proposed topology is more sensitive to the process variation than the original NMOS input transistor topology, because the zero bias noise condition depends of the NMOS - PMOS transconductance relation and the $C_{g s 2}$ $C_{d}$ capacitances relation. Corners simulations were performed for the MOS transistor model (PNMOS/NMOS: slow-slow slow-fast fast-slow fast-fast typ-typ), temperature $\left(0^{\circ}\right.$ and $\left.50^{\circ}\right)$ and voltage supply (1.1875V and $\left.1.325 \mathrm{~V}\right)$. The results of the ENC simulation corners for the proposed topology (PNMOS) and the NMOS input transistor topology ( $B_{02}=35 / 2, B_{03}=5 / 2, C_{B P}=560 \mathrm{pF}$ ) are presented in Figure 4.8. These simulation results show that the ENC variation of the proposed topology is acceptable compared with the original topology.

The zero bias noise condition demands a careful design to match the input transistor transconductance relation and the gate-source capacitance of $M_{2}$. Accordingly, another topology have been proposed in which the zero bias noise condition does not depend of $C_{g s 2}$ and a more flexible design is possible. This topology is studied in the next section. 


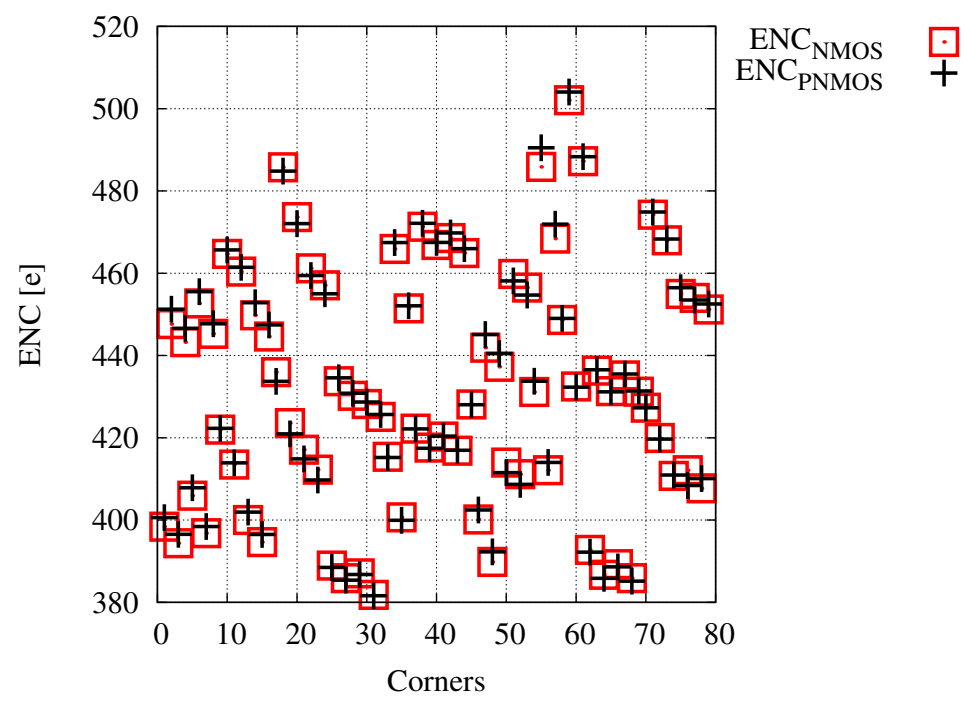

Figure 4.8: ENC simulation corners for the proposed topology (PNMOS) and the NMOS input transistor topology for $C_{d}=18.5 \mathrm{pF}, t_{s}=160 \mathrm{~ns}, S e n=30 \mathrm{mV} / \mathrm{fC}$ and $R_{s}=0 \Omega$.

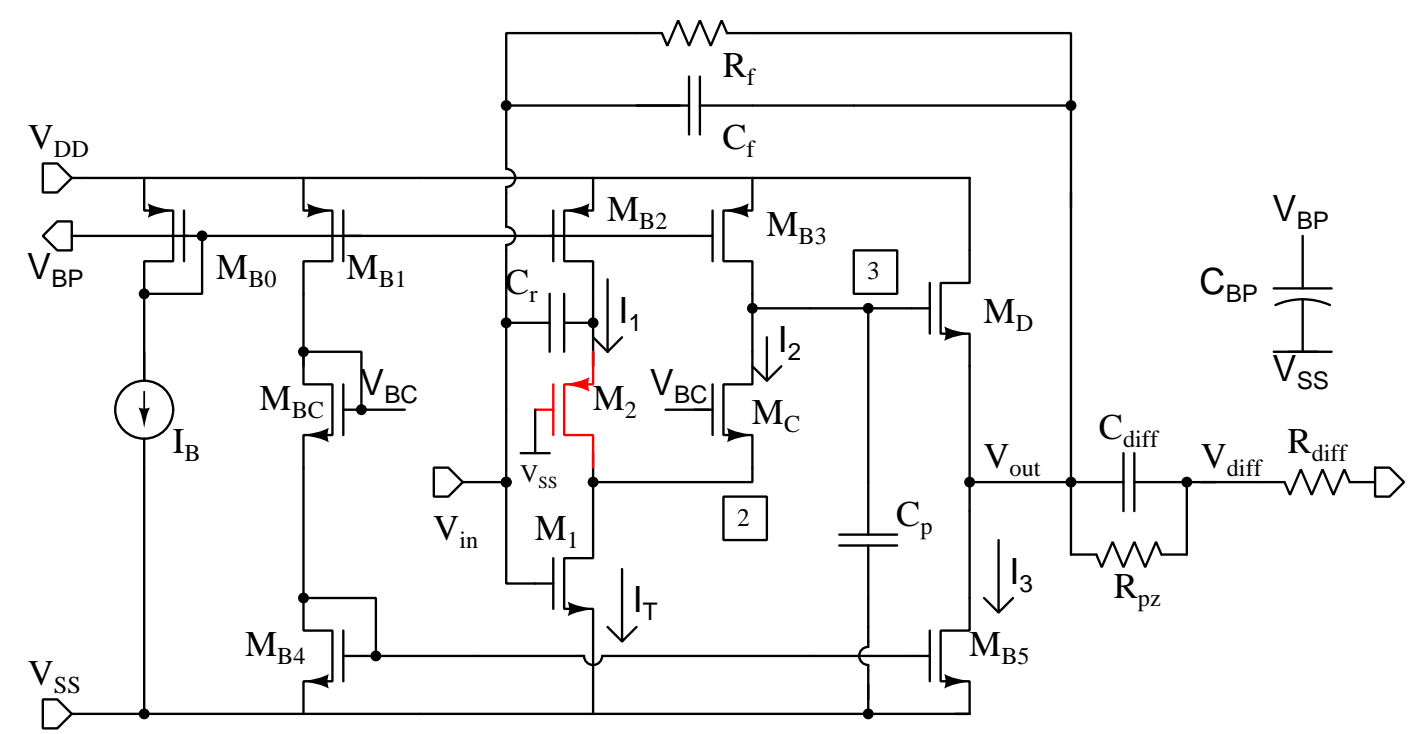

Figure 4.9: Schematic of the proposed $C_{r}$ feedback topology.

\section{2 $C_{r}$ feedback topology}

The schematic of the proposed new topology, called of $C_{r}$ feedback topology from this point, is presented in Figure 4.9. In this topology the $M_{2}$ transistor gate is not connected to the CSA input, instead the $M_{2}$ transistor gate is grounded and a $C_{r}$ capacitance is introduced, as illustrated in Figure 4.9. The small signal model of the considered topology is presented in Figure ??. In the same way as the $I_{B T}^{2}$ noise current referred to the amplifier input $\left(V_{i B T}^{2}\right)$ was calculated for the PMOS-NMOS input transistor topology, it 


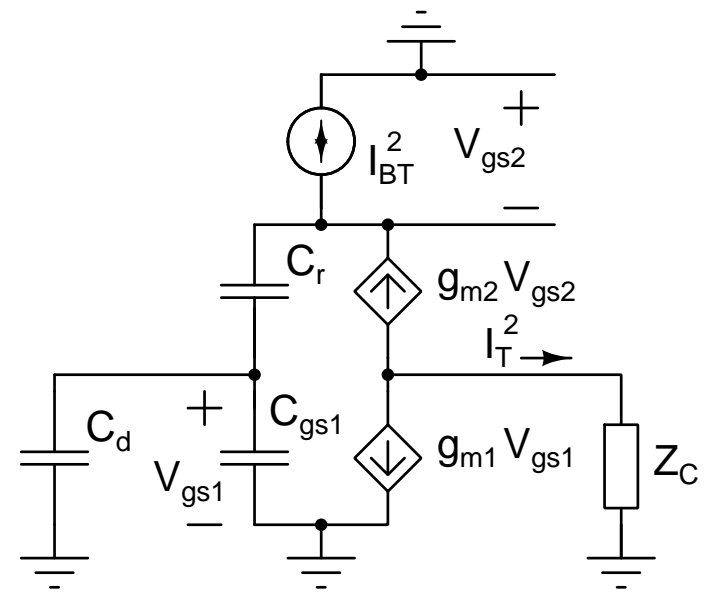

Figure 4.10: Small signal equivalent circuit of the $C_{r}$ feedback topology.

can be determined for $C_{r}$ feedback topology as follows:

$$
\begin{aligned}
& I_{T}^{2}=g_{m 2}^{2} V_{g s 2}^{2}-g_{m 1}^{2} V_{g s 1}^{2} \\
& V_{g s 1}^{2}=-\frac{C_{r}^{2}}{\left(C_{d}+C_{g s 1}\right)^{2}+C_{r}^{2}} V_{g s 2}^{2} \\
& V_{g s 1}^{2}=-\frac{C_{r}^{2}}{\left(C_{d}+C_{g s 1}\right)^{2}+C_{r}^{2}} V_{g s 2}^{2} \\
& -V_{g s 2}^{2}=\frac{I_{B T}^{2}}{s^{2}\left(C_{g s 2}^{2}+C_{r}^{2}\right)+g_{m 2}^{2}-\frac{s^{2} C_{r}^{2}}{\left(C_{d}+C_{g s 1}\right)^{2}+C_{r}^{2}}} \\
& \frac{I_{T}^{2}}{I_{B T}^{2}}=\frac{\frac{g_{m 1}^{2}}{g_{m 2}^{2}} \frac{C_{r}^{2}}{\left(C_{d}+C_{g s 1}\right)^{2}+C_{r}^{2}}-1}{s^{2}\left(C_{g s 2}^{2}+C_{r}^{2}-\frac{C_{r}^{2}}{\left(C_{d}+C_{g s 1}\right)^{2}+C_{r}^{2}}\right) \frac{1}{g_{m 2}^{2}}+1}
\end{aligned}
$$

Then, $\frac{I_{T}^{2}}{I_{B T}^{2}}$ is zero when:

$$
\frac{g_{m 1}^{2}}{g_{m 2}^{2}}=\frac{\left(C_{d}+C_{g s 1}\right)^{2}+C_{r}^{2}}{C_{r}^{2}}
$$

Considering $C_{g s 1}$ and $C_{r} l l C_{d}$ then $\left(C_{d}+C_{g s 1}\right)^{2}+C_{r}^{2} \approx C_{d}^{2}$. Therefore, the zero noise condition is defined by:

$$
\frac{g_{m 1}}{g_{m 2}} \approx \frac{C_{d}}{C_{r}}
$$




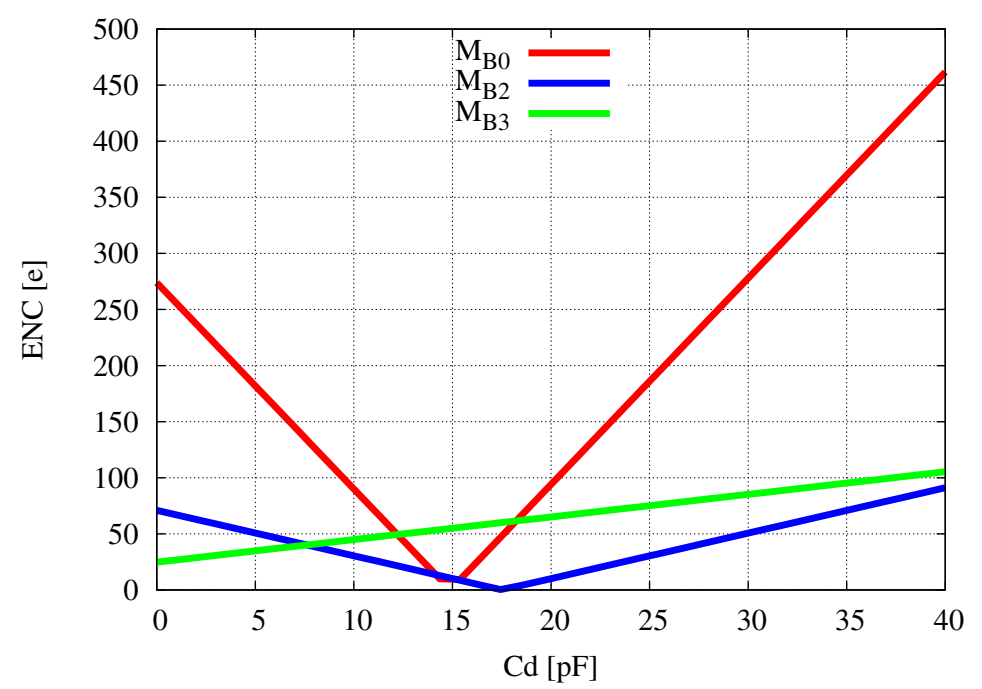

Figure 4.11: ENC components of $M_{B 0}, M_{B 2}$ and $M_{B 3}$ with $C_{r}$ feedback topology in relation to the detector capacitance for $t_{s}=160 \mathrm{~ns}, S e n=30 \mathrm{mV} / \mathrm{fC}$ and $R_{s}=0 \Omega$

The above equation determined the relation between $M_{1}$ and $M_{2}$ transconductance to cancel the bias noise for a specific detector capacitance value.

In addition to the bias noise cancellation effect of the $M_{2}$ transistor, it introduces an extra ENC component ( which can be determined by equation 4.10). The $M_{2}$ dimensions $(W L)_{2}$ and transconductance $\left(g_{m 2}\right)$ should be chosen so that its noise contribution be lower than the canceled noise component. Figure 4.11 shows the ENC component of $M_{B 0}, M_{B 2}$ and $M_{B 3}$ in relation to the detector capacitance of the proposed $C_{r}$ feedback topology for $C_{B P}=0 \mathrm{pF}, t_{s}=160 \mathrm{~ns}, S e n=30 \mathrm{mV} / \mathrm{fC}$ and $R_{s}=0 \Omega$. The CSA was designed for $C_{r}=1.85 \mathrm{pF}, g_{m 1}=44 \mathrm{mS}, g_{m 2}=4.4 \mathrm{mS}, C_{d}=18.5 \mathrm{pF}, I_{B}=100 \mu A, B_{02}=35 / 2$ and $B_{03}=5 / 2$. According to the equation (4.17) and the selected design parameters, the detector capacitance for zero bias noise condition is $18.5 \mathrm{pF}$ which is close to the schematic simulation results presented in Figure 4.11.

Corners simulations were performed for the MOS transistor model (PNMOS/NMOS: slow-slow slow-fast fast-slow fast-fast typ-typ), temperature $\left(0^{\circ}\right.$ and $\left.50^{\circ}\right)$ and voltage supply (1.1875V and $1.325 \mathrm{~V})$. The results of the ENC simulation corners for the proposed $C_{r}$ feedback topology and the NMOS input transistor topology $\left(B_{02}=35 / 2\right.$, $\left.B_{03}=5 / 2, C_{B P}=560 \mathrm{pF}\right)$ are presented in Figure 4.12. These simulations results show that the ENC variation of the proposed topology is acceptable compared with the original topology. 


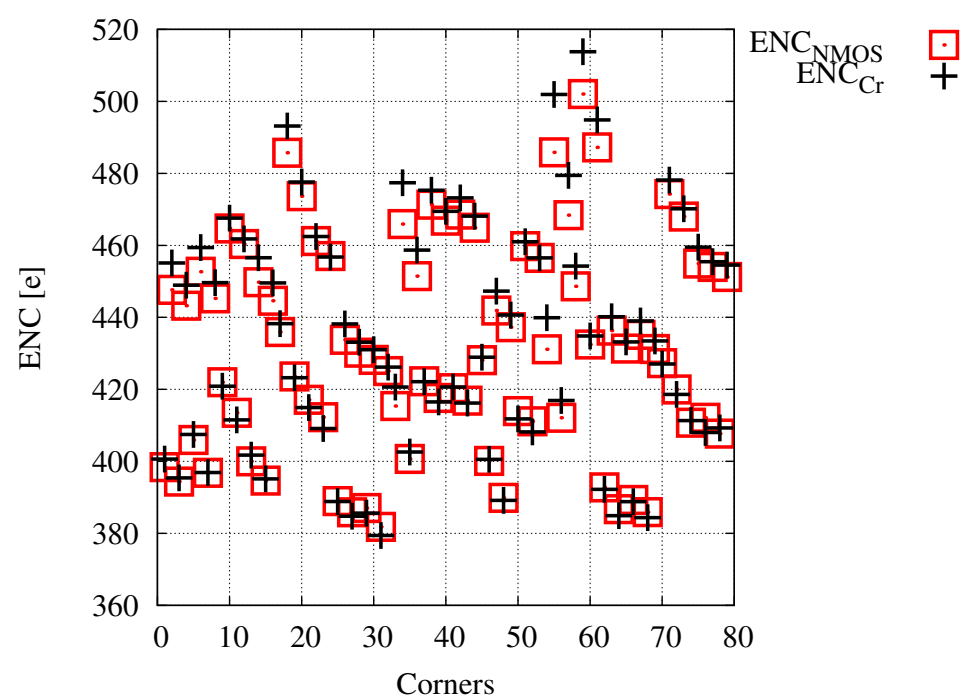

Figure 4.12: ENC simulation corners for the proposed $C_{r}$ feedback topology and the NMOS input transistor topology for $C_{d}=18.5 p F, t_{s}=160 \mathrm{~ns}, S e n=30 \mathrm{mV} / \mathrm{fC}$ and $R_{s}=0 \Omega$.

\subsection{Front-end PSRR improvement}

The ENC performance of the proposed topologies have been studied and them cancellation effect on bias noise components have been discussed in the previous section. The PSRR performance of the original and the proposed topologies will be analyzed in this section. This section starts with an analyze of the front-end PSRR.

\subsubsection{Front-end PSRR}

The Front-end PSRR $\left(P S R R_{F E}\right)$ can be approximated from cascade connection analysis of the CSA and the Semi-gaussian pulse shaper by the following equation defined in [STEYAERT; SANSEN, 1990]:

$$
\frac{1}{P S R R_{F E}}=\frac{1}{\operatorname{PSRR}_{C S A}}-\frac{1}{\operatorname{Gain}_{C S A} A}+\underbrace{\frac{\operatorname{Gain}_{V_{D D}}^{\text {Shaper }}}{\operatorname{Gain}_{C S A} \text { Gain }_{\text {Shaper }}(s)}}_{\text {part III }}
$$

where $\operatorname{Gain}_{C S A}$ and $\operatorname{Gain}_{S h a p e r}(s)$ are the gain of the CSA and the pulse shaper, respectively, and $\operatorname{Gain}_{V_{D D}}^{S h a p e r}$ is the signal gain from the power supply of the pulse shaper. The equation (4.18) indicates that the front-end PSRR is defined by the CSA PSRR and it is deteriorated by the CSA gain and the $\operatorname{Gain}_{\text {Shaper }}(s) \operatorname{Gain}_{C S A}$ product: the front-end PSRR is reduced when the pulse shaper gain is increased for a specific CSA gain. 


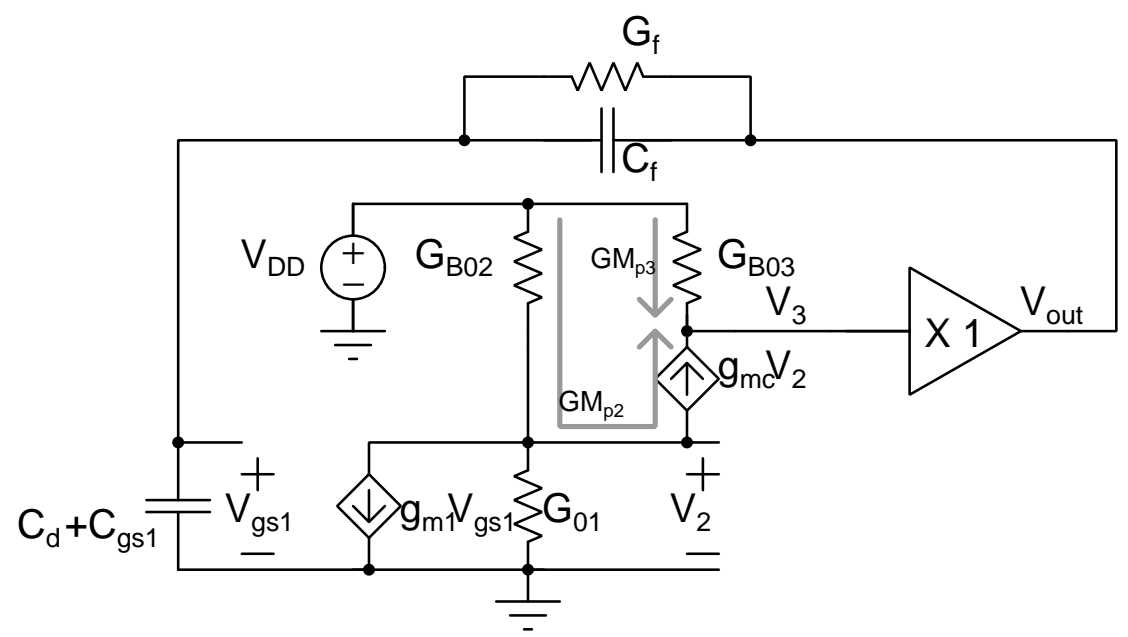

Figure 4.13: Simplified small signal model for the PSRR study if the CSA with the original topology

The equation 4.18 part III has a high impact on the front-end PSRR at the pulse shaper frequency band, because the pulse shaper gain rises around this frequency band, i.e the front-end PSRR decreases if the front-end sensitivity increases.

\subsubsection{PSRR of the CSA original topology}

The simplified small signal model for the PSRR study if the CSA with the original topology is presented in Figure 4.13. In this model, the parasitic capacitance of the amplifier have been disconsidered because the amplifier dominant pole is at higher frequencies than the pulse shaper band frequency (from $1 \mathrm{MHz}$ to $3 \mathrm{MHz}$ for $t_{s}=160 \mathrm{~ns}$ ), and the output resistance of the $M_{C}$ was also not included. $G_{01}, G_{0 B 2}$ and $G_{0 B 3}$ correspond to the output conductance of $M_{1}, M_{B 2}$ and $M_{B 3}$, respectively. $G_{f}$ is the CSA feedback transconductance. The PSRR component generated by the voltage follower output stage was disconsidered, and it have been substituted by a ideal unity gain buffer.

From analysis of the Figure 4.13 circuit, the following equations can be defined:

$$
\begin{gathered}
\left(V_{2}-V_{D D}\right) G_{0 B 2}+V_{2} G_{01}+g_{m C} V_{2}+V_{3} \frac{s C_{d}+G_{f}}{s\left(C_{d}+C_{f}\right)+G_{f}} g_{m 1}=0 \\
\left(V_{3}-V_{D D}\right) G_{0 B 3}-g_{m C} V_{2}=0
\end{gathered}
$$

From equations (4.19) and (4.20) $V_{3}$ is given by: 


$$
V_{3}=\frac{G_{0 B 3} V_{D D}+\frac{G_{0 B 2} g_{m C} V_{D D}}{g_{m C}+G_{0 B 2}+G_{01}}}{\left.G_{0 B 3}+\frac{\left(s C_{f}+G_{f}\right) g_{m} g_{m 1}}{\left[g_{m C}+G_{0 B 2}+G_{01}\right]\left[s\left(C_{d}+C_{g s}+C_{f}\right)+G_{f}\right.}\right]}
$$

Assuming $g_{m C} \gg G_{0 B 2}+G_{01}$, then PSSR referred to the output is described by:

$$
\operatorname{PSRR}_{C S A}=\frac{V_{D D}}{V_{\text {out }}}=\frac{s\left[\left(C_{f}+C_{d}+C_{g s 1}\right) G_{0 B 3}+C_{f} g_{m 1}\right]+\left(G_{0 B 3} G_{f}+g_{m 1} G_{f}\right)}{\left(G_{0 B 3}+G_{0 B 2}\right)\left[s\left(C_{d}+C_{f}+C_{g s 1}\right)+G_{f}\right]}
$$

Assuming $g_{m 1} \gg G_{0 B 3}$ the previous equation is:

$$
\begin{aligned}
P S R R_{C S A} & =\frac{s C_{f} g_{m 1}+g_{m 1} G_{f}}{\left(G_{0 B 3}+G_{0 B 2}\right)\left[s\left(C_{d}+C_{f}+C_{g s 1}\right)+G_{f}\right]} \\
P S R R & =\underbrace{\frac{g_{m 1}}{G_{0 B 3}+G_{0 B 2}}}_{\text {part I }} \cdot \underbrace{\frac{\left({ }^{2} \frac{C_{f}}{G_{f}}+1\right)}{\left.s \frac{C_{d}+C_{f}+C_{g s 1}}{G_{f}}+1\right)}}_{\text {part II }}
\end{aligned}
$$

Important conclusions can be made from equation (4.24):

- Equation (4.24) Part I corresponds to the PSRR of the amplifier which can be demonstrate by a simple analysis of the amplifier circuit, which the PSRR can be alternatively defined by the ratio of the signal transconductance $(G M)$ and the power supply transconductance $\left(G M_{p}\right)$ as presented in [STEYAERT; SANSEN, 1990].

- The transconductance from power supply to the amplifier output has 2 components illustrated in Figure 4.13 as $G M_{p 2} \approx G_{0 B 2}$ and $G M_{p 3} \approx G_{0 B 3}$.

- The CSA PSRR can be defined by the amplifier PSRR (equation 4.24 part I) and a CSA transfer function ( equation 4.24 part II).

- The power supply transconductance of the amplifier has low components generated by the current paths illustrated in 4.13 .

- For the pulse shaper frequency band the PSRR is deteriorated by the transfer function pole $\frac{G_{f}}{C_{d}+C_{f}+C_{g s 1}}$ and the zero $\frac{G_{f}}{C_{f}}$ as shown in Figure 4.14. 


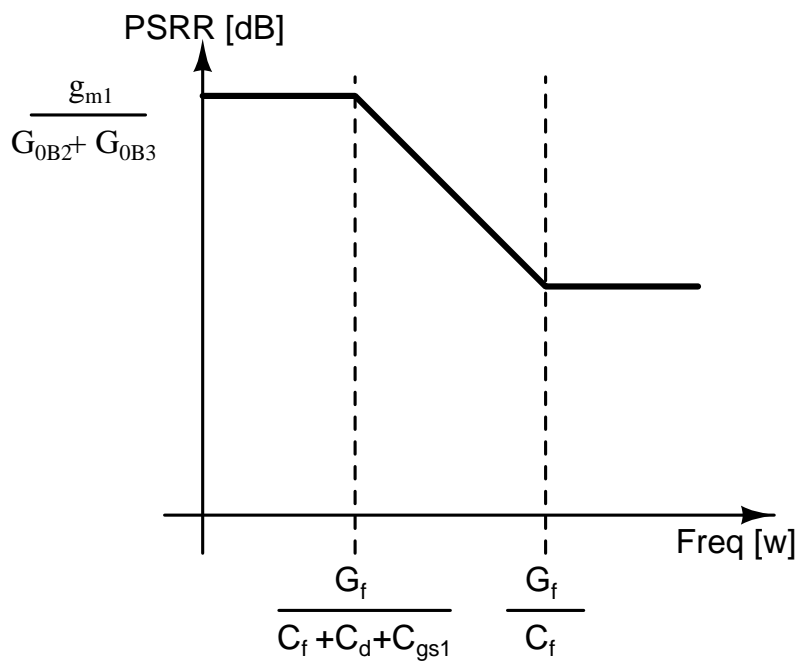

Figure 4.14: Theoretical PSRR from equation (4.24).

- The $P S R R_{C S A}$ at high frequency is deteriorated if the detector capacitance is increased.

- The transfer function pole and zero are mutually canceled when $C_{d}=0 \mathrm{pF}$ (the transfer function pole and zero are very close).

The $P S R R_{C S A}$ referred to the output simulation of the designed CSA with NMOS input transistor topology for $C_{d}=0 \mathrm{pF}-20 \mathrm{pF}-40 \mathrm{pF}, C_{f}=1.6 \mathrm{pF}, G_{f}=1 / 5 \mathrm{M} \Omega, g_{m 1}=44 \mathrm{mS}$, $G_{0 B 2}=119 \mu S$ and $G_{0 B 2}=17 \mu S$ is presented in Figure 4.15. From equation (4.24) the PSRR theoretical is $50.2 \mathrm{~dB}$ for $\mathrm{s}=0$, result that is close to the value obtained from simulation. The $P S R R_{C S A}$ curve showed in accordance to the expected theoretical behavior presenting one pole and one zero, which are mutually canceled when $C_{d}=0 \mathrm{pF}$. The simulation and the theoretical $P S R R_{C S A}$ at $2 \mathrm{MHz}$ versus the detector capacitance for $t_{s}=160 \mathrm{~ns}$ and $S e n=30 \mathrm{mV} / \mathrm{fC}$ is presented in Figure 4.16 . $2 \mathrm{MHz}$ correspond to the central frequency of the pulse shaper for $t_{s}=160 \mathrm{~ns}$. These results validate the PSRR analysis presented in this work.

A simulation of the $P S R R_{F E}$ referred to the output with the NMOS input transistor topology for $C_{d}=18.5 \mathrm{pF}, t_{s}=160 \mathrm{~ns}, S e n=30 \mathrm{mV} / \mathrm{fC}$ and $36 \mathrm{~dB}, 32.5 \mathrm{~dB}$ and $24 \mathrm{~dB}$ of pulse shaper gain, is shown in Figure 4.17. This simulation results are close to the expected theoretical behavior described by equation (4.18). 


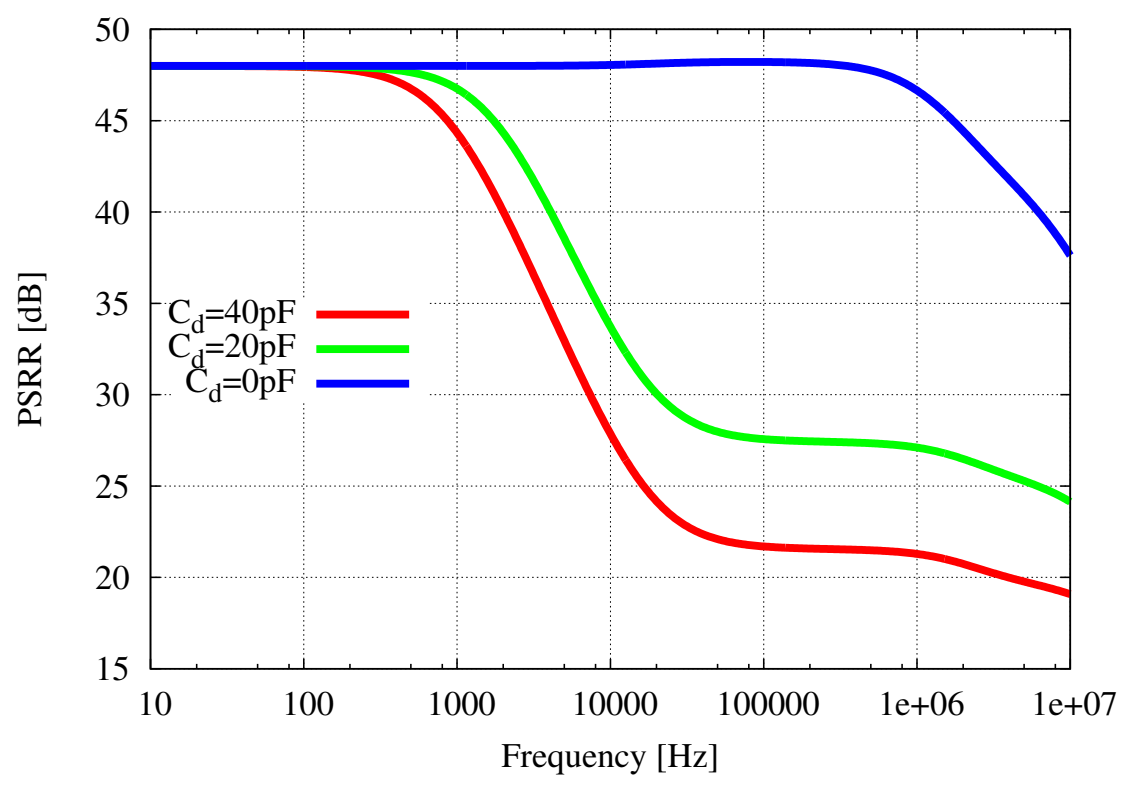

Figure 4.15: $P S R R_{C S A}$ simulation of the designed CSA with the NMOS input transistor topology for $C_{d}=0 \mathrm{pF}-20 \mathrm{pF}-40 \mathrm{pF}, C_{f}=1.6 \mathrm{pF}, G_{f}=1 / 3 \mathrm{M} \Omega, g_{m 1}=44 \mathrm{mS}, G_{0 B 2}=119 \mu S$ and $G_{0 B 2}=17 \mu S$.

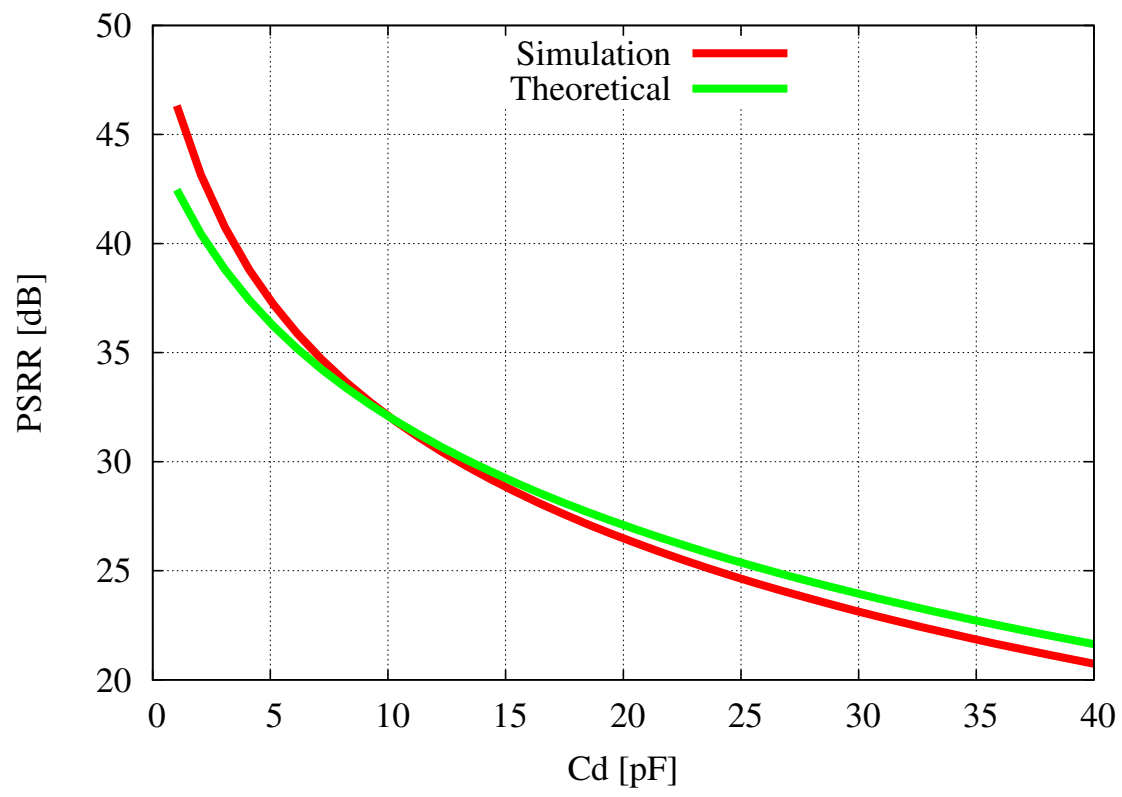

Figure 4.16: $P S R R_{C S A}$ at $2 \mathrm{MHz}$, simulation and the theoretical value versus the detector capacitance. 


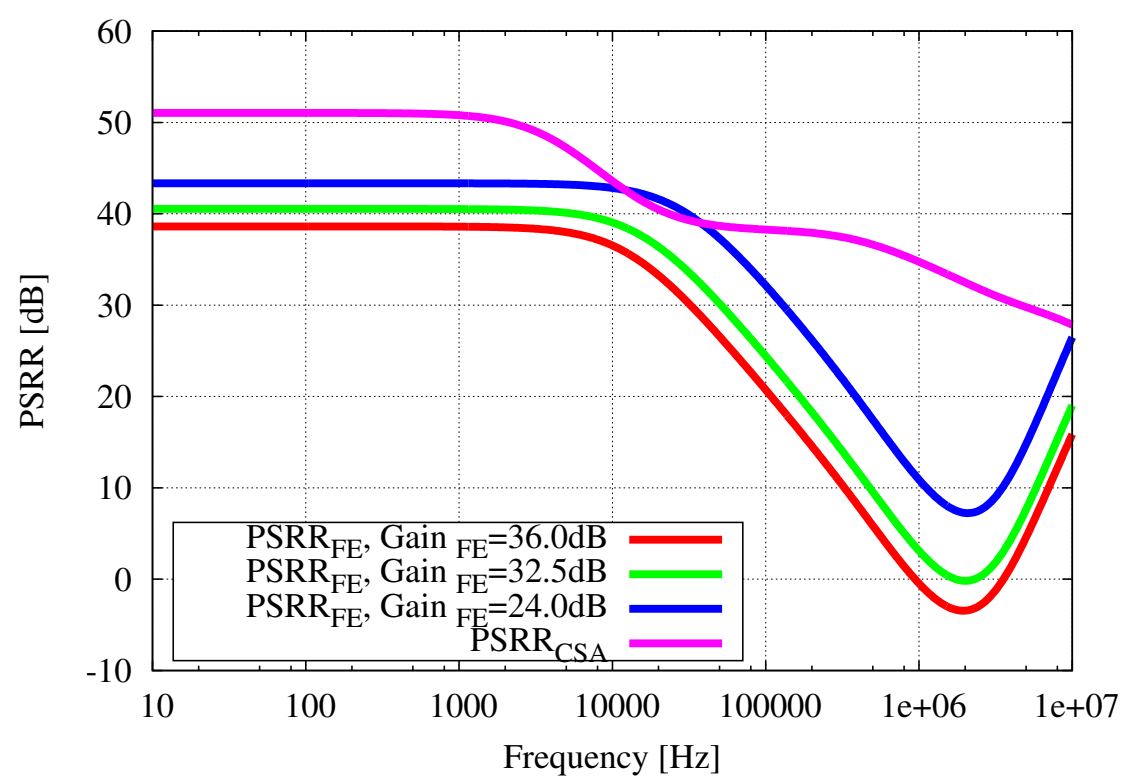

Figure 4.17: Simulation of the $P S R R_{F E}$ with the NMOS input transistor topology for $C_{d}=18.5 \mathrm{pF}, t_{s}=160 \mathrm{~ns}, S e n=30 \mathrm{mV} / \mathrm{fC}$ and $36 \mathrm{~dB}, 32.5 \mathrm{~dB}$ and $34 \mathrm{~dB}$ of front-end gain 


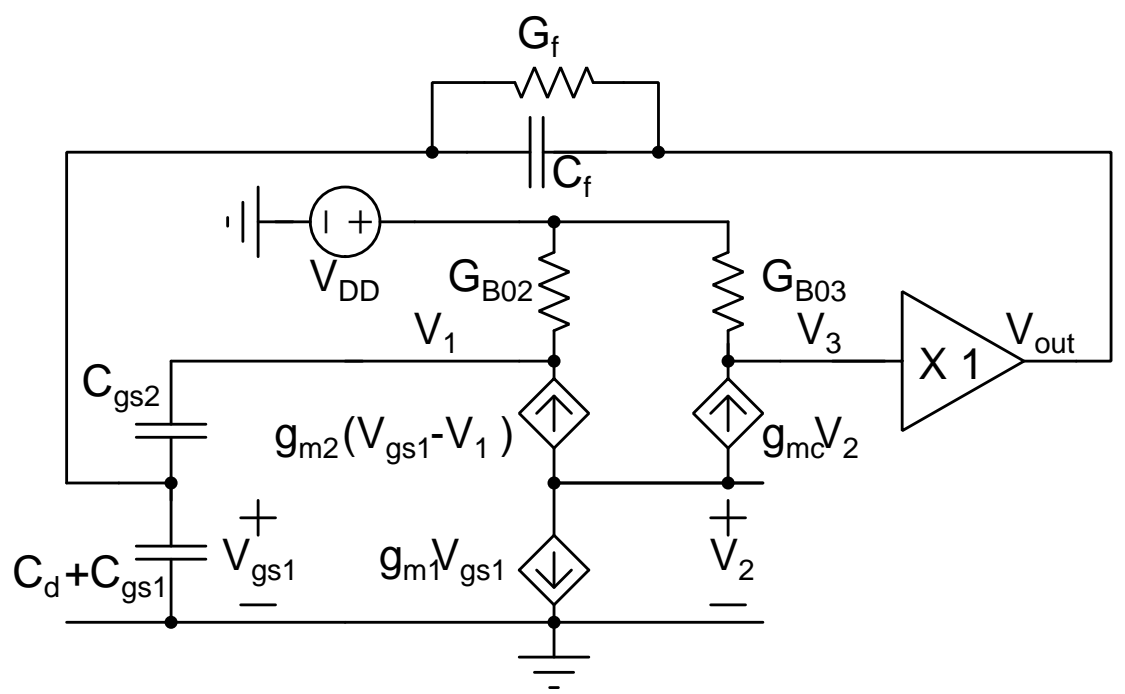

Figure 4.18: Small signal model shown for the PSRR analysis of the PMOS-NMOS input transistor topology.

\subsubsection{PSRR of the PMOS-NMOS input transistor topology}

The previous PSRR analysis can be easily extrapolated for the PNMOS-NMOS input transistor topology. A PSRR expression for the proposed topology can be determined from the small signal model shown in Figure 4.18. The equations used in this study are:

$$
\begin{gathered}
s\left(C_{d}+C_{g s 1}\right) V_{g s 1}+s C_{g s 2}\left(V_{g s 1}-V_{1}\right)+\left(V_{g s 1}-V_{3}\right)\left(s C_{f}+G_{f}\right)=0 \\
\left(V_{1}-V_{D D}\right) G_{0 B 2}-g_{m 2}\left(V_{1}-V_{g s 1}\right)+s C_{g s 2}\left(V_{1}-V_{g s 1}\right)=0 \\
g_{m 2}\left(V_{1}-V_{g s 1}\right)+g_{m C} V_{2}+g_{m 1} V_{g s 1}=0 \\
\left(V_{3}-V_{D D}\right) G_{0 B 3}-g_{m C} V_{2}=0
\end{gathered}
$$

Assuming $g_{m 1} \gg g_{m C} \gg G_{0 B 2}+G_{01}+G_{01}, g_{m 2} \gg g_{m C} \gg G_{0 B 2}+G_{01}+G_{01}$ and $C_{t}=C_{g s 1}+C_{d}$ a PSRR referred to the output expression for the PNMOS-NMOS input transistor topology can be obtained and it is given by: 


$$
\begin{aligned}
& P S R R=\frac{s C_{f} g_{m 1} g_{m 2}+g_{m 1} g_{m 2} G_{f}}{s\left[-C_{g s 2} G_{0 B 2} g_{m 1}+g_{m 2}\left(C_{t} G_{0 B 2}+\ldots\right.\right.} \\
& \overline{\left.\left.C_{t} G_{0 B 3}+C_{f} G_{0 B 2}+C_{f} G_{0 B 3}\right)\right]+\left(G_{0 B 2} g_{m 2} G_{f}+G_{0 B 3} g_{m 2} G_{f}\right)} \\
& P S R R=\underbrace{\frac{g_{m 1}}{G_{0 B 3}+G_{0 B 2}}}_{\text {part I }}
\end{aligned}
$$

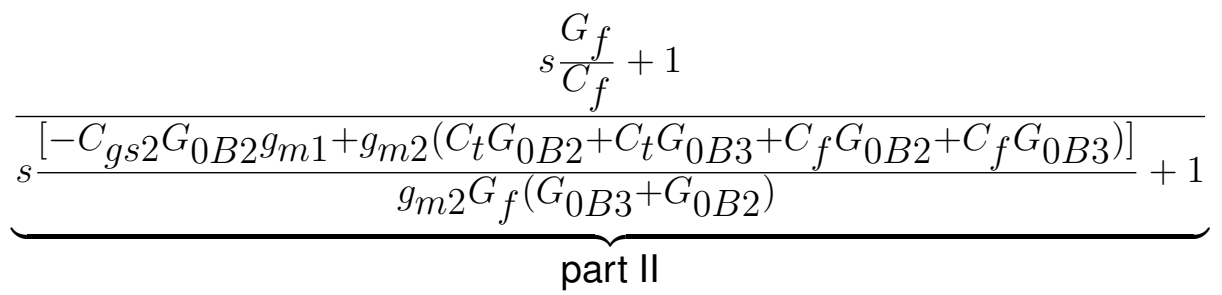

Clearly, the above equation is similar to equation (4.24) in which the PSRR is the product of the amplifier PSRR and the CSA transfer function. The difference between equations (4.24) and (4.30) is the transfer function pole. Now, the pole depend of the detector capacitance and it can be eliminated if $C_{g s 2} G_{0 B 2} g_{m 1}=g_{m 2}\left(C_{t} G_{0 B 2}+\right.$ $\left.C_{t} G_{0 B 3}+C_{f} G_{0 B 2}+C_{f} G_{0 B 3}\right)$. The elimination of this pole will avoid the PSRR degradation at pulse shaper band frequencies. In the CSA design was chosen $B_{02}=35 / 2$ and $B_{03}=5 / 2$, so it is possible to assume $G_{0 B 2} \gg G_{0 B 3}$ and a simple relation for the transfer function pole cancellation can be obtained:

$$
\frac{g_{m 1}}{g_{m 2}}=\frac{C_{d}+C_{g s 1}+C_{f}}{C_{g s 2}}
$$

The equation(4.31) is the same zero bias noise condition found in section (4.1) assuming $C_{d}+C_{g s 1}+C_{f} \approx C_{d}^{2}$. This analysis demonstrates that the proposed PNMOS-NMOS topology should bring superior PSRR.

The PSRR referred to the output simulation of the designed CSA with PMOS-NMOS input transistor topology for $C_{d}=0 \mathrm{pF}-13.5 \mathrm{pF}-15.3 \mathrm{p}-40 \mathrm{pF}, C_{f}=1.6 \mathrm{pF}, G_{f}=1 / 5 \mathrm{M} \Omega, g_{m 1}=$ $44 \mathrm{mS}, g_{m 2}=10 \mathrm{mS}, C_{g s 2}=4.2 \mathrm{pF}, G_{0 B 2}=119 \mu S$ and $G_{0 B 2}=17 \mu S$ is presented in Figure 4.19. From equation (4.30) the PSRR theoretical is $50.2 \mathrm{~dB}$ at $0 \mathrm{~Hz}$, result that is close to the value obtained from simulation. The PSRR curve showed is in accordance to the expected theoretical behavior presenting one pole $\left(p_{1}\right)$ and one zero $\left(z_{1}\right)$, which are 


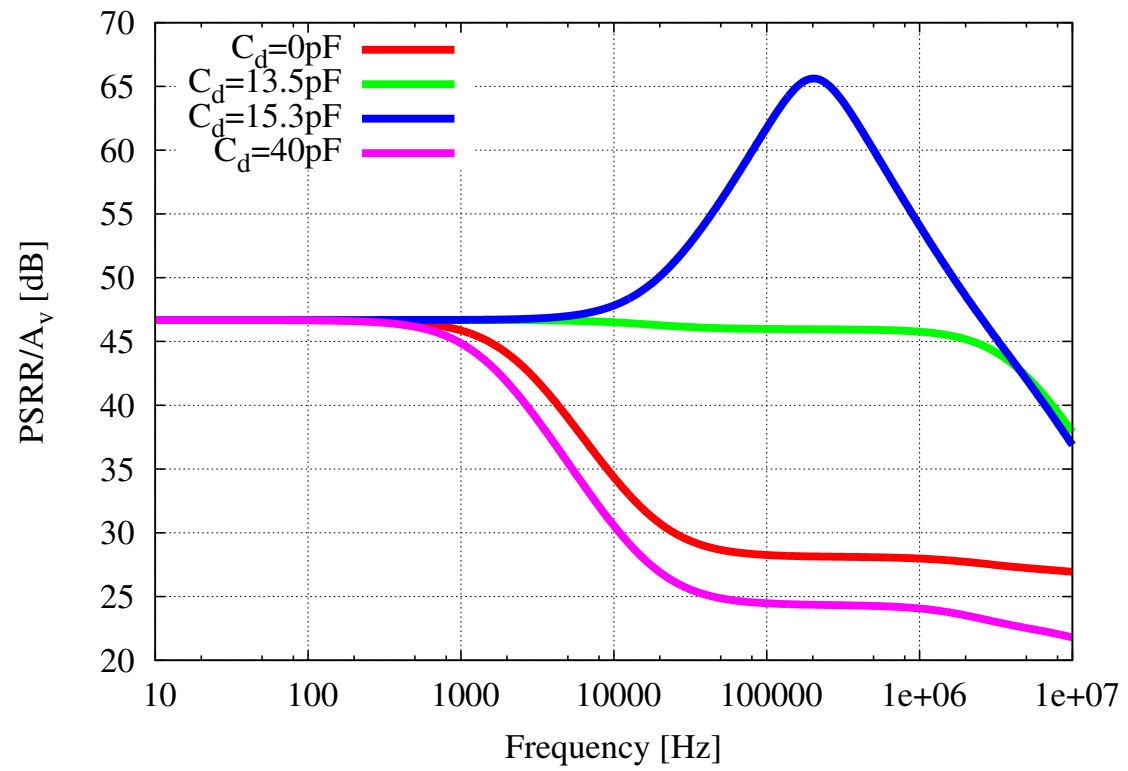

Figure 4.19: PSRR simulation of the designed CSA with PMOS-NMOS input transistor topology for $C_{d}=0 \mathrm{pF}-20 \mathrm{pF}-40 \mathrm{pF}, C_{f}=1.6 \mathrm{pF}, G_{f}=1 / 3 \mathrm{M} \Omega, g_{m 1}=44 \mathrm{mS}, G_{0 B 2}=119 \mu S$ and $G_{0 B 2}=17 \mu S$.

mutually canceled when the detector capacitance is (equation 4.30):

$$
C_{d}=\frac{C_{f}\left(G_{0 B 3}+G_{0 B 2}\right)}{G_{0 B 2}}-C_{g s 1}-C_{f}+\frac{C_{g s 2} g_{m 1}}{g_{m 2}}
$$

From the above equation the theoretical detector capacitance for $p_{1}=z_{1}$ is $17.8 \mathrm{pF}$, value compatible with the simulation value $(13.5 \mathrm{pF})$ shown in Figure 4.19. The simulation and the theoretical PSRR at $2 \mathrm{MHz}$ versus the detector capacitance is presented in Figure 4.20. These results validate the PSRR analysis presented in this work. 


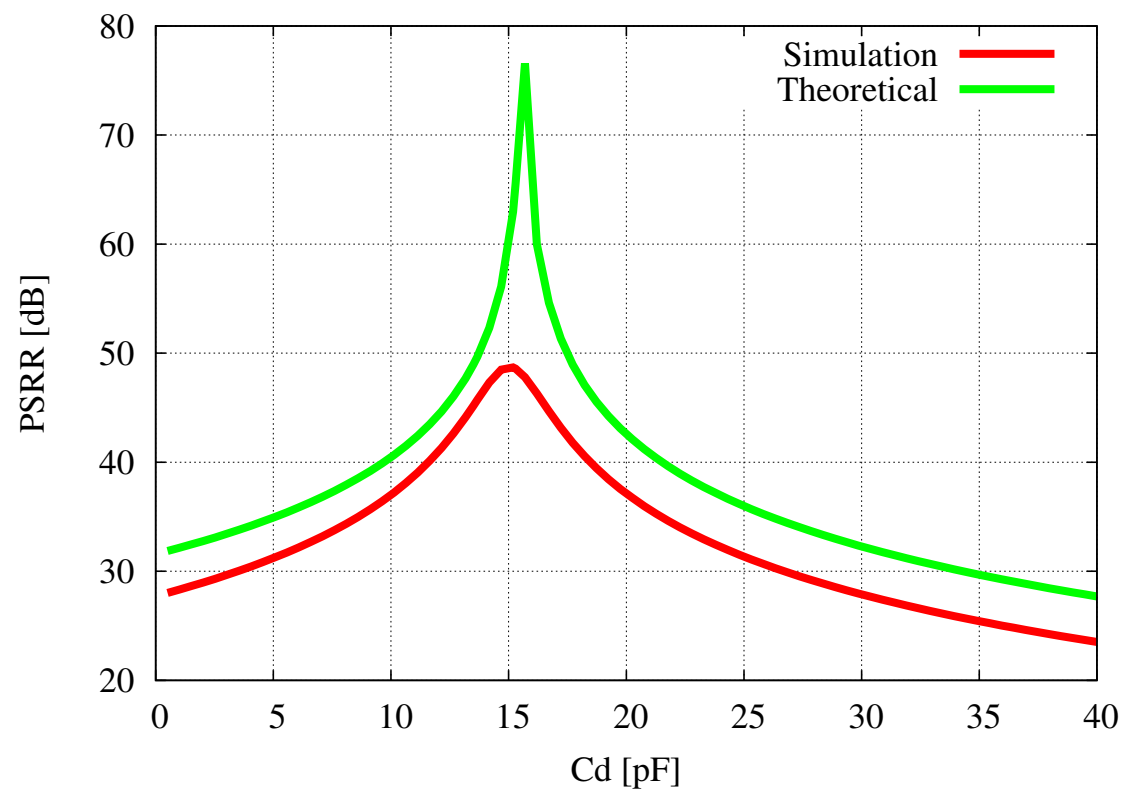

Figure 4.20: PSRR simulation and the theoretical PSRR at $2 \mathrm{MHz}$ versus the detector capacitance for the designed CSA with PMOS-NMOS input transistor topology.

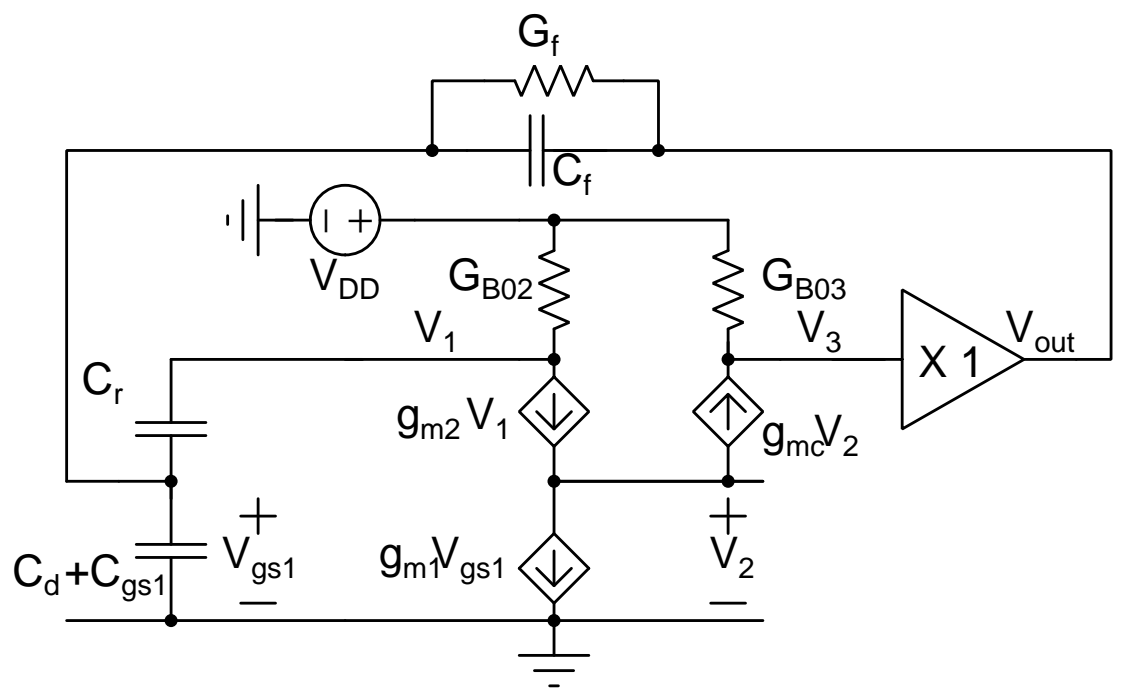

Figure 4.21: Small signal model shown for the PSRR analysis of the $C_{r}$ feedback topology.

\subsubsection{PSRR of the $C_{r}$ feedback topology}

The previous PSRR analysis can be easily extrapolated for the $C_{r}$ feedback topology. A PSRR referred to the output expression for the proposed topology is given can be determined from the small signal model shown in Figure 4.21. The equations used in this analysis are:

$$
s\left(C_{d}+C_{g s 1}\right) V_{g s 1}+s C_{g s 2}\left(V_{g s 1}-V_{1}\right)+\left(V_{g s 1}-V_{3}\right)\left(s C_{f}+G_{f}\right)=0
$$




$$
\begin{gathered}
\left(V_{1}-V_{D D}\right) G_{0 B 2}+g_{m 2} V_{1}+s C_{r}\left(V_{1}-V_{g s 1}\right)+s C_{g s 2} V_{1}=0 \\
-g_{m 2} V_{1}+g_{m C} V_{2}+g_{m 1} V_{g s 1}=0 \\
\left(V_{3}-V_{D D}\right) G_{0 B 3}-g_{m C} V_{2}=0
\end{gathered}
$$

Assuming $g_{m 1} \gg g_{m C} \gg G_{0 B 2}+G_{01}+G_{01}, g_{m 2} \gg g_{m C} \gg G_{0 B 2}+G_{01}+G_{01}$ and $C_{t}=C_{g s 1}+C_{d}$ a PSRR referred to the output expression for the $C_{r}$ feedback topology can be obtained and it is given by:

$$
\begin{aligned}
& P S R R=\frac{s C_{f} g_{m 1} g_{m 2}+g_{m 1} g_{m 2} G_{f}}{s\left[-C_{g s 2} G_{0 B 2} g_{m 1}+g_{m 2}\left(C_{t} G_{0 B 2} \cdots\right.\right.} \\
& \overline{\left.\left.+C_{t} G_{0 B 3}+\left(C_{f}-C_{r}\right) G_{0 B 2}+\left(C_{f}-C_{r}\right) G_{0 B 3}\right)\right]+\left(G_{0 B 2} g_{m 2} G_{f}+G_{0 B 3} g_{m 2} G_{f}\right)} \\
& P S R R=\underbrace{\frac{g_{m 1}}{G_{0 B 3}+G_{0 B 2}}}_{\text {part I }} \\
& \underbrace{\frac{{ }^{s} \frac{G_{f}}{C_{f}}+1}{s \frac{\left[-C_{g s 2} G_{0 B 2} g_{m 1}+g_{m 2}\left(C_{t} G_{0 B 2}+C_{t} G_{0 B 3}+\left(C_{f}+C_{r}\right) G_{0 B 2}+\left(C_{f}+C_{r}\right) G_{0 B 3}\right)\right]}{g_{m 2} G_{f}\left(G_{0 B 3}+G_{0 B 2}\right)}+1}}_{\text {part II }}
\end{aligned}
$$

Clearly, the above equation is similar to equation (4.30) in which the PSRR is the product of the amplifier PSRR and a transfer function (part II). The transfer function pole can be eliminated if the following condition is achieved:

$$
C_{g s 2} G_{0 B 2} g_{m 1}=g_{m 2}\left(C_{t} G_{0 B 2}+C_{t} G_{0 B 3}+\left(C_{f}+C_{r}\right) G_{0 B 2}+\left(C_{f}+C_{r}\right) G_{0 B 3}\right)
$$

Similarly, assuming $G_{0 B 2} \gg G_{0 B 3}$ a simple relation for the transfer function pole cancellation can be obtained:

$$
\frac{g_{m 1}}{g_{m 2}}=\frac{C_{d}+C_{g s 1}+C_{f}}{C_{r}}
$$




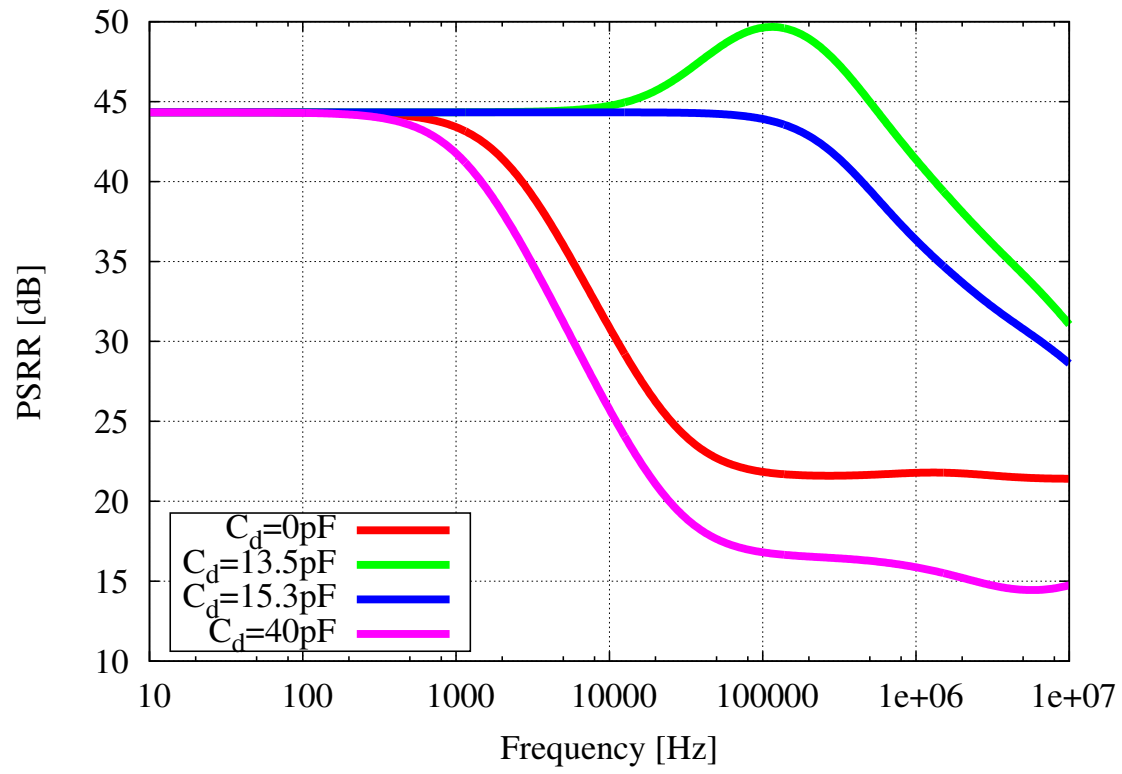

Figure 4.22: PSRR simulation of the designed CSA with $C_{r}$ feedback topology for $C_{d}=0 \mathrm{pF}-$ 20pF-40pF, $C_{f}=1.6 \mathrm{pF}, G_{f}=1 / 3 \mathrm{M} \Omega, g_{m 1}=44 \mathrm{mS}, G_{0 B 2}=119 \mu S$ and $G_{0 B 2}=17 \mu S$.

The equation (4.40) is the same zero bias noise condition found in section (4.2) for the $C_{r}$ feedback topology. This analysis demonstrates that the proposed $C_{r}$ feedback topology is also superior in terms of PSRR than the NMOS input transistor topology.

The PSRR referred to the output simulation of the designed CSA with $C_{r}$ feedback topology for $C_{d}=0 \mathrm{pF}-13.5 \mathrm{pF}-15.3 \mathrm{p}-40 \mathrm{pF}, C_{f}=1.6 \mathrm{pF}, G_{f}=1 / 5 \mathrm{M} \Omega, g_{m 1}=44 \mathrm{mS}, g_{m 2}=$ $4.4 \mathrm{mS}, C_{r}=1.85 \mathrm{pF}, G_{0 B 2}=119 \mu S$ and $G_{0 B 2}=17 \mu S$ is presented in Figure 4.22. The PSRR curve showed in accordance to the expected theoretical behavior presenting one pole $\left(p_{1}\right)$ and one zero $\left(z_{1}\right)$, which are mutually canceled when the detector capacitance is (equation 4.38):

$$
\frac{C_{f}\left(G_{0 B 3}+G_{0 B 2}\right)}{G_{0 B 2}}-C_{g s 1}-C_{f}+\frac{C_{r} g_{m 1}}{g_{m 2}}
$$

From the above equation the theoretical detector capacitance for $p_{1}=z_{1}$ is $17.8 \mathrm{pF}$, value compatible with the simulation value (13.5pF) shown in Figure 4.22.

A comparison of the PSRR at $2 \mathrm{MHz}$ of the designed CSA with the NMOS input transistor topology ( $P S R R_{N M O S}$ ), with the PMOS-NMOS input transistor topology and with the $C_{r}$ feedback topology $\left(P S R R_{C_{r}}\right)$ is presented in Figure 4.23 for $t_{s}=160 \mathrm{~ns}$ and Sen $=30 \mathrm{mV} / \mathrm{fC}$. This result shows a PSRR improvement of $16 \mathrm{~dB}$ for $18.5 \mathrm{pF}$ of detector capacitance. 


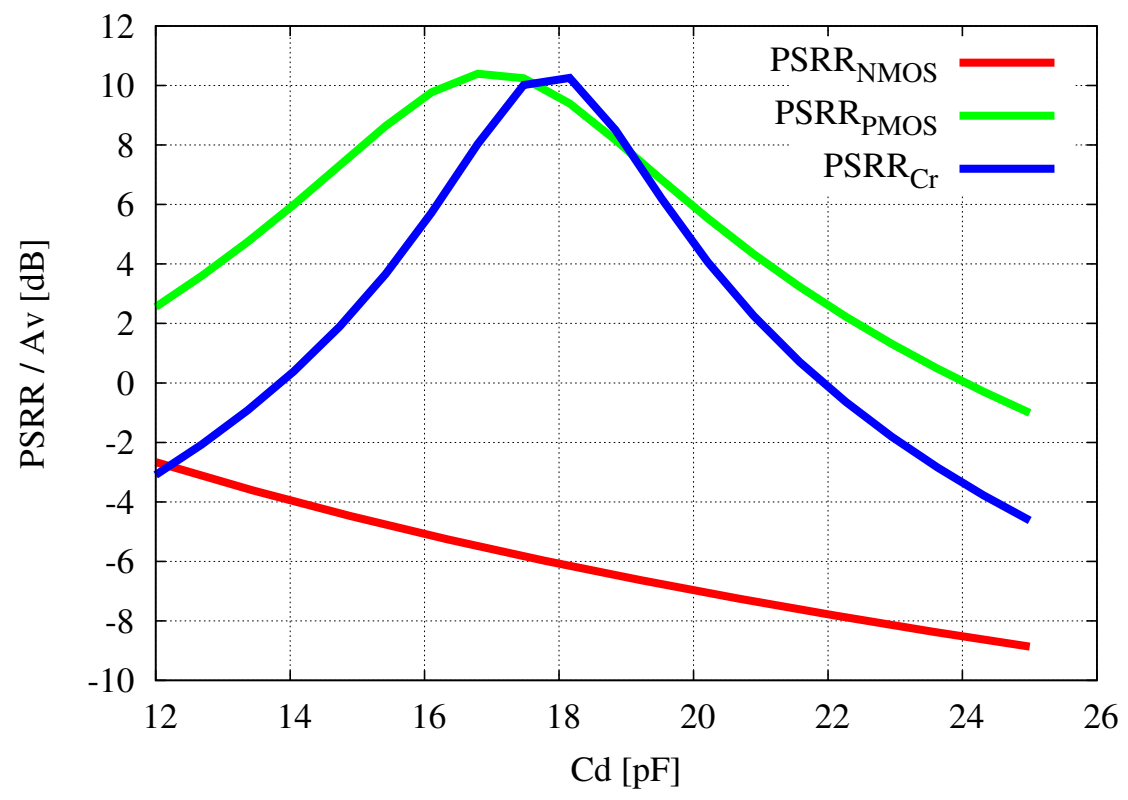

Figure 4.23: Comparison of the PSRR at $2 \mathrm{MHz}$ of the designed CSA with the NMOS input transistor topology $\left(P S R R_{N M O S}\right)$, with the PMOS-NMOS input transistor topology and with the $C_{r}$ feedback topology for $t_{s}=160 \mathrm{~ns}$ and $S e n=30 \mathrm{mV} / \mathrm{fC}$. 


\section{Chapter 5}

\section{Experimental results}

A chip of the designed front-end was fabricated in 130nm TSMC CMOS technology with 6 Metal layers (the top metal was thick metal) via IMEC Blelgium, called the SAMPA chip01 from this point. The produced prototype contains 5 channels of the front-end block with independent inputs/outputs per channel and common control signals, , as shown in Figure 5.1. In summary, the front-end block is composed of a positive/negative polarity Charge Sensitive Amplifier (CSA) and a shaper, which transform the charge signal into a differential semi-Gaussian voltage signal. The Charge Sensitive Amplifier (CSA) has a capacitive feedback $C_{f}$ and a resistive feedback $R_{f}$ connected in parallel, a Pole-Zero Cancellation (PZC) network, a high pass filter, two bridged-T second order low pass filters, a non-inverting stage. The first shaper is a scaled-down version of the CSA and generates the first two poles and one zero. A copy of the first shaper connected in unity gain configuration is implemented in order to provide a differential mode input to the next stage. The second stage of the shaper is a fully differential second order bridged-T filter and it includes a Common-Mode Feed-Back network (CMFB). The non-inverting stage adapts the DC voltage level of the shaper output to use the full dynamic range of the ADC. It consists of a parallel connection of two equally designed Miller compensated amplifiers and an output RC low pass filter $(\mathrm{R}=50 \Omega, \mathrm{C}=20 \mathrm{pF})$. The output front-end resistance is typically $50 \Omega$. $0 \Omega$ of ESD resistence was chosen for this fist prototype. 


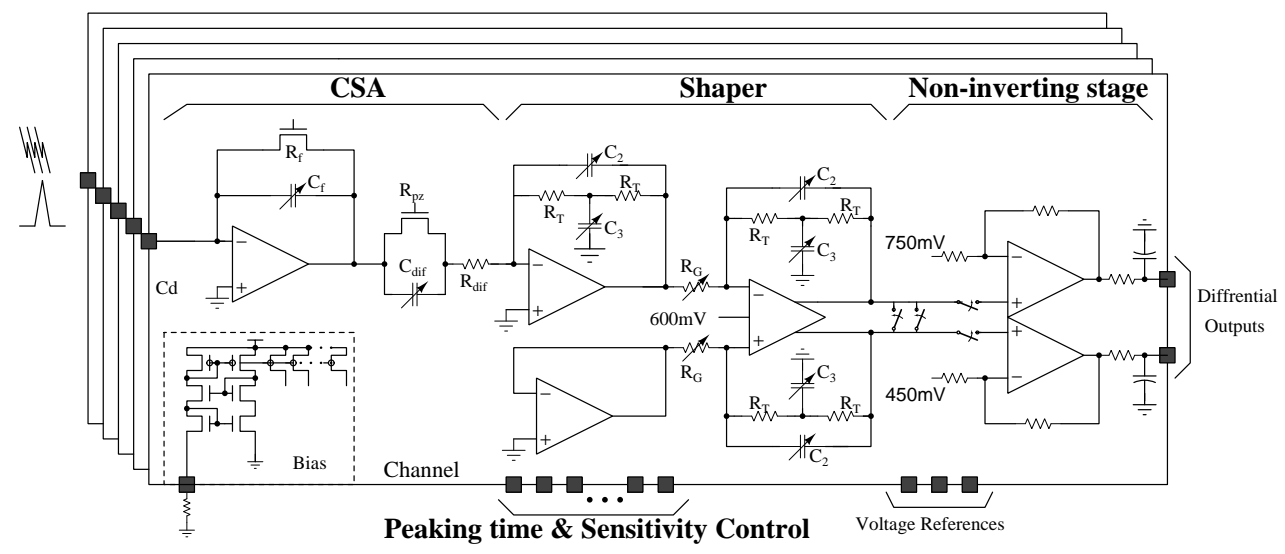

Figure 5.1: Block diagram of the 5 front-end channels chip.

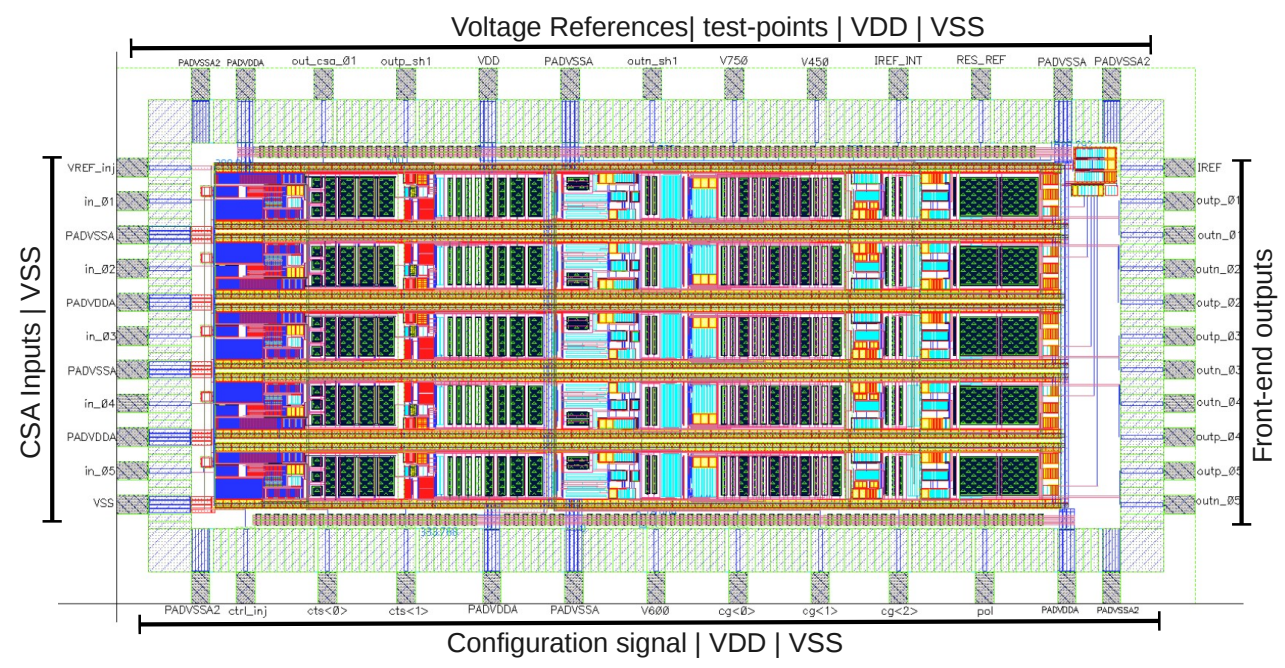

Figure 5.2: Layout of the 5 front-end channels chip.

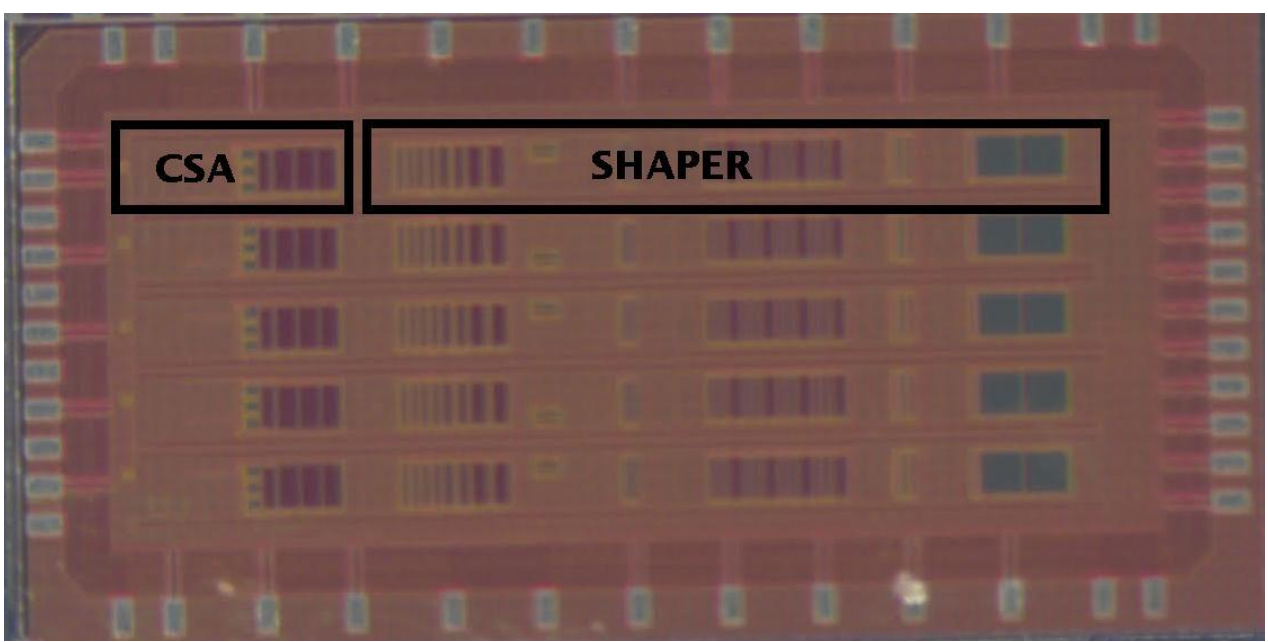

Figure 5.3: Micro-photograph of the fabricated chip with 5 channels of the designed front-end in 130nm TSMC CMOS technlogy.

\subsection{Description of the SAMPA chip01}

The chip layout and the pin distribution is shown in Figure 5.2. To avoid parasitic crosstalk components a ground pad separates the CSA inputs between adjacent chan- 
Table 5.1: Peakinng time configurations of the SAMPA chip01.

\begin{tabular}{|c|c|c|}
\hline Peaking time & cts $<0>$ & cts $<1>$ \\
\hline $80 n s$ & low & low \\
\hline $160 n s$ & high & low \\
\hline $300 n s$ & high & high \\
\hline
\end{tabular}

Table 5.2: Sensitivity configurations of the SAMPA chip01.

\begin{tabular}{|c|c|c|c|}
\hline Sensitivity & $\mathrm{cg}<0>$ & $\mathrm{cg}<1>$ & $\mathrm{cg}<2>$ \\
\hline $30 \mathrm{mV} / \mathrm{fC} @ 160 \mathrm{~ns}$ & low & low & low \\
\hline $20 \mathrm{mV} / \mathrm{fC} @ 160 \mathrm{~ns}$ & high & low & low \\
\hline $30 \mathrm{mV} / \mathrm{fC} @ 80 \mathrm{~ns}$ & low & high & low \\
\hline $20 \mathrm{mV} / \mathrm{fC} @ 80 \mathrm{~ns}$ & high & high & low \\
\hline $4 \mathrm{mV} / \mathrm{fC} @ 300 \mathrm{~ns}$ & low & low & high \\
\hline
\end{tabular}

nels. Also, the CSA input were placed on the left side and the Front-end output were placed on the right side. 5 VDD pins were distributed on the top and bottom side, the $600 \mathrm{mV}, 450 \mathrm{mV}$ and $750 \mathrm{mV}$ reference voltage pins were placed on the top sside, and the peaking time and sensitivity configuration pins on the bottom side. Metal 6 (thick metal) was used to route the voltage supply and ground around the chip blocks to minimize the metal lines resistance. The CSA output and the first shaper stage output of the first front-end channel are still accessible through dedicated pads for testing purpose. A micro-photograp of the produced chips is shown in Figure 5.3.

The chip nominal supply voltage is $1.25 \mathrm{~V}$, with variation range of $\pm 5 \%$ leading to $1.1875 \mathrm{~V}<1.25 \mathrm{~V}<1.325 \mathrm{~V}$. In order to cover all the specifications for the ALICE experiment, the chip can be configured to operate on 3 peaking time options controlled by cts $<0: 1>$ pins, 5 sensitivity options controlled by $c g<0: 2>$ pins and on negative/positive charge input polarity controlled by $\mathrm{pol}$ pin. The Tables $5.1,5.2,5.3$ summarize the possible chip configurations (high $=V_{D D}$, low $=V_{S S}$ ). The 5 front-end channels chip has a total of 62 input/output pads and it was packaged in CQFP64. A test board was designed and fabricated in order to test the packaged SAMPA chip01. A board photographic is presented in Figure 5.4. 
Table 5.3: Polarity configurations of the SAMPA chip01p.

\begin{tabular}{|c|c|}
\hline Polarity & pol \\
\hline Positive & low \\
\hline Negative & high \\
\hline
\end{tabular}

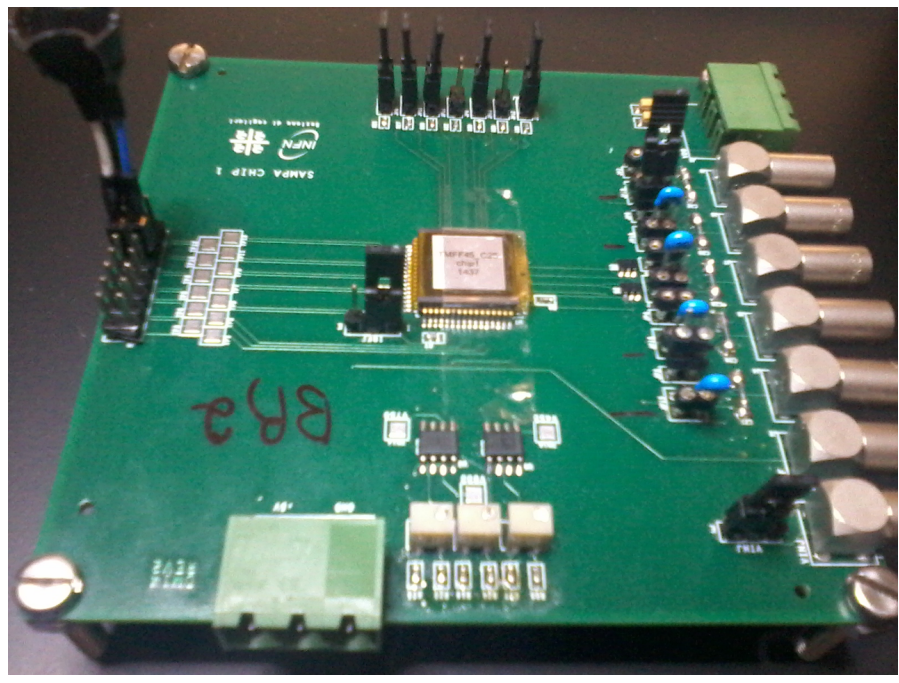

Figure 5.4: Photographic of the test board used for experimental testing of the packaged SAMPA chip01.

\subsection{Experimental Set-up of the SAMPA chip01 test}

The test system set-up is presented in Figure 5.5. The charge input pulse was generated by a capacitor where a terminal is connected to the chip input and the other terminal is connected to a step-up/down signal generator (pulse generator). An external $1.8 \mathrm{pF}$ capacitor was used to inject the charge for all the measurements. The fall or rise edge of the pulse signal sets the charge injection polarity. Positive polarity correspond to rise edge and negative polarity to fall edge. The rise/fall time of the step signal should be much higher than the minimum peaking time specification. Values lower than 2 ns must be selected. An on-board $50 \Omega$ resistance in parallel with the pulse generator input was included for impedance matching. The injected charge can be calculated by the following equation:

$$
\text { Charge } \approx \Delta \text { Voltage } \cdot \text { Capacitance }
$$

The $1.25 \mathrm{~V}$ nominal supply voltage $\left(V_{D D}\right)$ is provided by an external power supply generator. An external on-board capacitor, set in parallel to the input, was used to simulate 


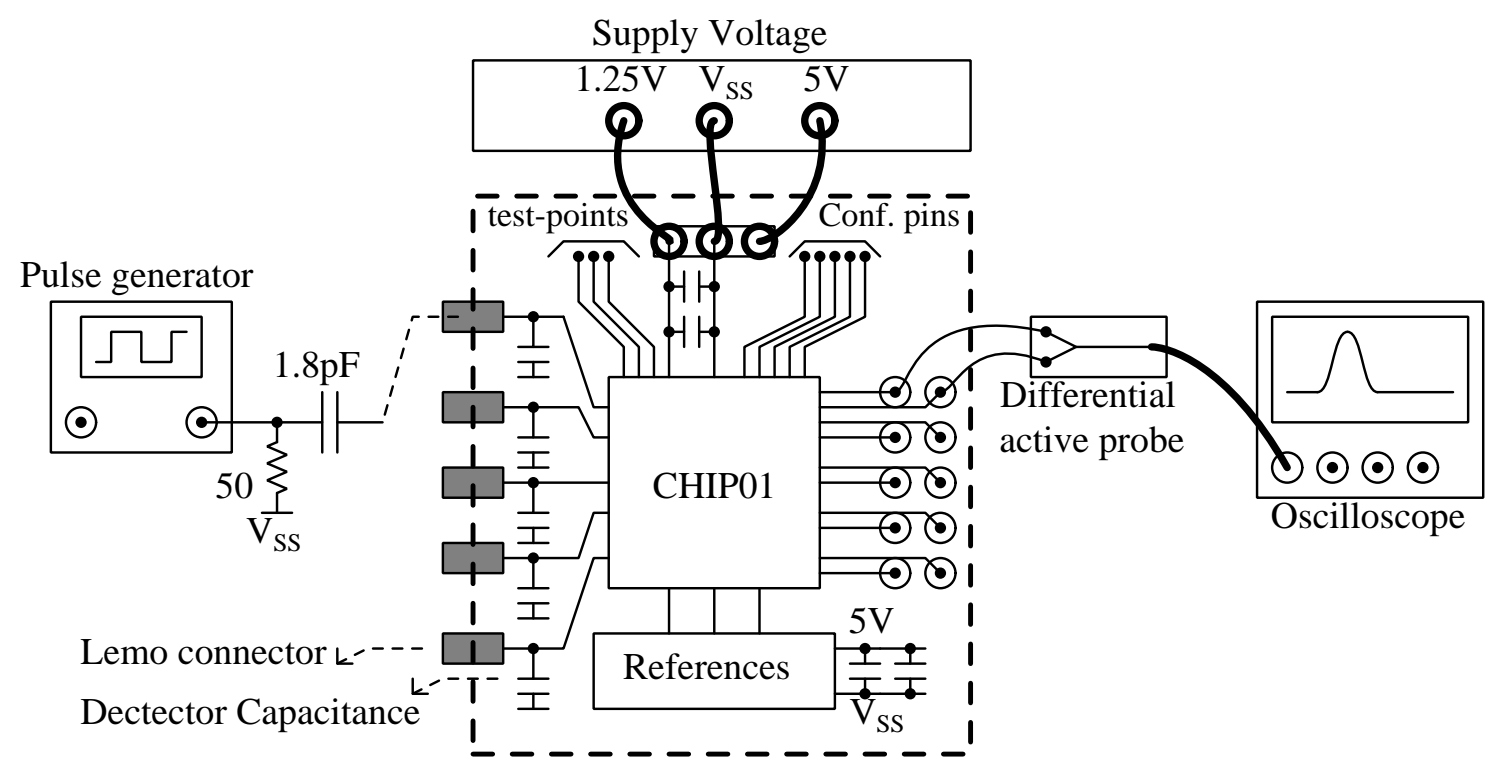

Figure 5.5: Test system set-up for the peaking time and sensitivity measurements.

the detector capacitance. The printed circuit board together with the chip package showed a measured parasitic capacitance to ground equal to $15 \mathrm{pF}$. This parasitic capacitance was added to the input capacitance value in the experimental results. A lemo connector was used to couple the charge signal generated by the $1.8 \mathrm{pF}$ capacitor to the front-end input. The chip differential output signal are connected to a pin header and a differential active probe with $200 k \Omega$ of input resistance and $700 \mathrm{fF}$ of input capacitance is used to acquire the pulsing signal with the oscilloscope. The technical characteristics of the equipments used in the experimental SAMPA chip01 test are summarized in Table 5.4. The reference voltages of the front-end are generated by a resistive trimmable circuit included in the implemented test board. The schematic of the used circuit based on the TLV2772 chip (High Slew Rate Rail-To-Rail Output Operational Amplifier) is shown in Figure 5.6. This circuit provides a low output impedance which is necessary to avoid transient variation of the voltage reference when the front-end is pulsing. The consequence of transient variations of the front-end voltage reference will be an extra crosstalk component common for all channels. An extra power supply of $5 \mathrm{~V}$ was included for the TLV2772 chip's supply. $10 \mu \mathrm{F}$ and 100nF decoupling capacitors were used for noise and $A C$ components reduction of the power supply placed very close to the chip. Decoupled ground and supply voltage planes were designed on the board with the purpose to provide a low-impedance ground connection and reduce EMI (Electromagnetic interference) effects. 
Table 5.4: Technical characteristics of the equipments used in the experimental SAMPA chip01 test.

\begin{tabular}{|c|c|}
\hline Oscilloscope & Keysight DSOS204A 2GHz, 20/10 GSa/s, 10bit native \\
\hline Voltage supply & Computer controllable, Keithley 2200-20-5 \\
\hline Waveform Generator & $120 \mathrm{MHz}, 2$-Channel - Keysight 3362A2 Trueform \\
\hline
\end{tabular}

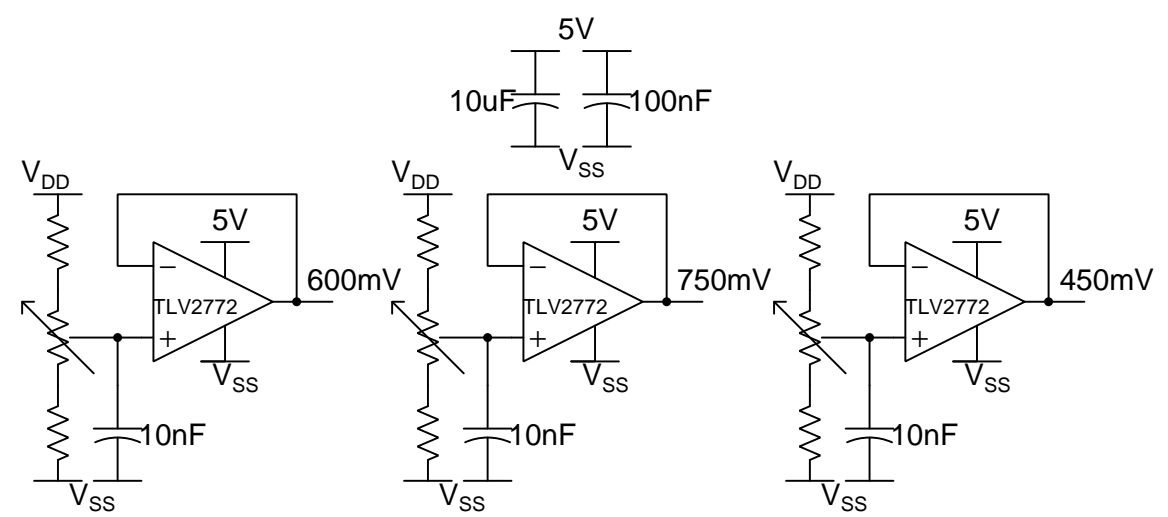

Figure 5.6: Schematic of the resistive trimmable circuit used to generate the front-end voltage references.

\subsubsection{Polarization and Power consumption}

This test allowed to verify the DC voltages levels when the chip was correctly polarised. There are two ways to supply the chip bias current: (a) connecting the reference pin IREF to an external voltage set on the test board or (b) using an internal current source generated on the chip. The way (a) was used in this test for $160 \mathrm{~ns}$ of peaking time, $30 \mathrm{mV} / \mathrm{fC}$ of sensitivity, and positive polarity configuration. The DC voltages were measured and showed an agreement with the expected values from the simulation. The power consumption was measured using a Source Meter and the power consumption per channel is calculated by dividing the total power consumption by the number of channels. The expected typical total power consumption of the 5 front-end channels chip is $45.6 \mathrm{~mW}$ for $V_{D D}=1.25(9.1 \mathrm{~mW}$ per channel). The average value of the measured power for the produced prototypes was $44 \mathrm{~mW}$.

\subsubsection{Output pulse specifications}

To measure the pulse shape, a voltage step signal was applied to the $1.8 \mathrm{pF}$ injection capacitor in series with the chip input. This test measured the front-end specifications of peaking time and sensitivity. Figure 5.7 shows a sample of the analog output pulses 
measured at the shaper output for the different sensitivity (Sen) and peaking time $\left(t_{s}\right)$ configurations, several values of input charge $\left(Q_{i n}\right)$ and $V_{D D}=1.25 \mathrm{~V}$. The pulse shape was also measured as a function of the detector capacitance and $50 \mathrm{fC}$ of input charge, as show in Figure 5.8. The typical non-linearity specifications of the 5 front-end channels chip is lower than $1 \%$. After the chip is correctly polarized, 100 charge values were injected linearly and the output pulse amplitude was measured. The INL specification is estimated as the maximum deviation of any of the acquired points from the straight line (drawn by using the method of least square from the acquired data). Figure 5.9 shows a sample of the output peak voltage ( Vout ), the linear fit $\left(\right.$ Vout $\left._{\text {fit }}\right)$ and the INL[\%] as function of the input charge for the 3 sensitivity cases and $V_{D D}=1.25 \mathrm{~V}$. The circuit presented a INL[\%] lower than $1 \%$ to an input charge of $100 \mathrm{fC}$ for the $20 \mathrm{mV} / \mathrm{fC}$ configuration, $67 \mathrm{fC}$ for the $30 \mathrm{mV} / \mathrm{fC}$ configuration and $500 \mathrm{fC}$ for the $4 \mathrm{mV} / \mathrm{fC}$ configuration, for an detector capacitance of $20 \mathrm{pF}$ and $1.1875 \mathrm{~V}-1.325 \mathrm{~V}$ of voltage supply. This measure was performed for 3 chips and they have showed that the experimental circuit linearity is within the specification limits.

For the $4 \mathrm{mV} / \mathrm{fC}$ positive/negative configuration, the sensitivity of the channel 2 and 5 of a chip sample shown in Figure 5.10(a) . They were measured for detector capacitances up to $180 \mathrm{pF}$ and compared with the simulation results. Figure 5.10 (b) shows the measured and simulated peaking time versus detector capacitance for the same sensitivity case case. The measurement shows a weak dependence on the detector capacitance. The simulated peaking time for the circuit is about 290ns.

For the $20 \mathrm{mV} / \mathrm{fC}$ and $30 \mathrm{mV} / \mathrm{fC}$ configurations, samples of the sensitivities are shown in Figures 5.11(a) and 5.12(a) measured for detector capacitance up to 40pF and $V_{D D}=1.25 \mathrm{~V}$. These results shows good uniformity within $5 \%$ of variation. Figures 5.11 (b) and 5.12(b) show the measured and simulated peaking time versus detector capacitance. The measurement shows a peaking time of $137 \mathrm{~ns}$ at $30 \mathrm{pF}$ input capacitance and a strong dependence on the detector capacitance. The expected peaking time from the electrical simulation is about 160ns. The discrepancy between measurements and simulations is probably due to a metallization option mistake during the chip submission which changed the MIM capacitor parameters.

For the $20 \mathrm{mV} / \mathrm{fC}$ and $30 \mathrm{mV} / \mathrm{fC}$ configurations, samples of the sensitivities are shown in Figures 5.13(a), 5.14(a) and 5.15(a) measured for input charge up to maximum in- 
put charge, $15 \mathrm{pF}$ of detector capacitance and $V_{D D}=1.25 \mathrm{~V}$. Figures $5.13(\mathrm{~b}), 5.14(\mathrm{~b})$ and 5.15 (b) show the measured peaking time versus input charge for the same measure set-up. 


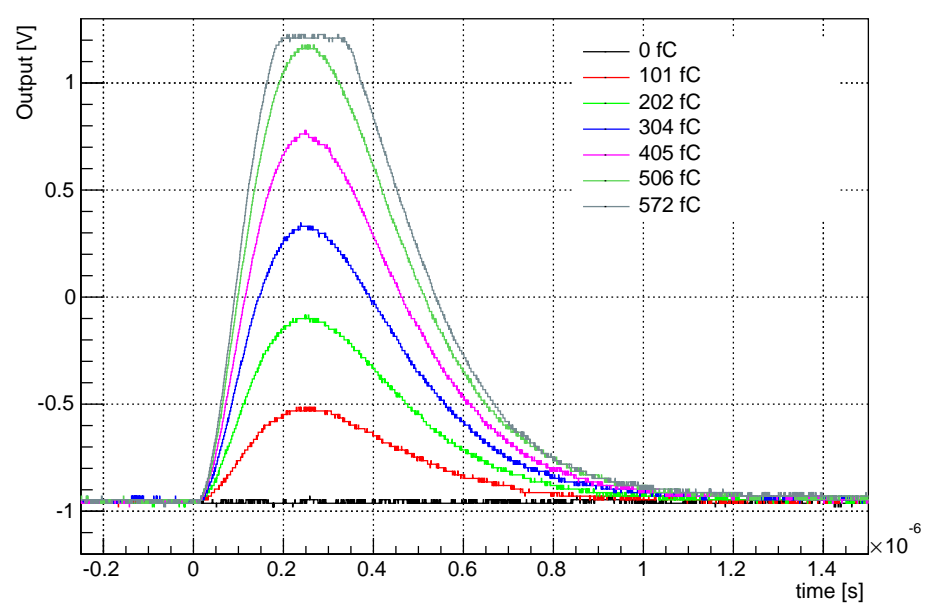

(a) $\mathrm{Sen}=4 \mathrm{mV} / \mathrm{fC}$ (pos), $t_{s}=300 \mathrm{~ns}, Q_{i n}=0 \mathrm{C}$ to $572 \mathrm{fC}, \mathrm{Cd}=20 \mathrm{pF}$.

Pulse

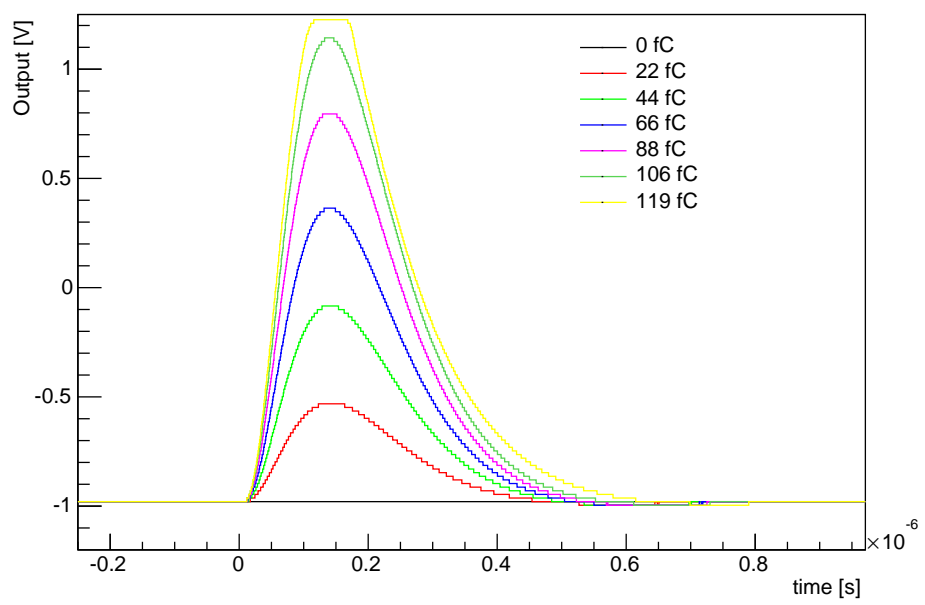

(b) Sen=20mV/fC (neg), $t_{s}=160 \mathrm{~ns}, Q_{i n}=0 \mathrm{C}$ to $119 \mathrm{fC}, \mathrm{Cd}=20 \mathrm{pF}$.

Pulse

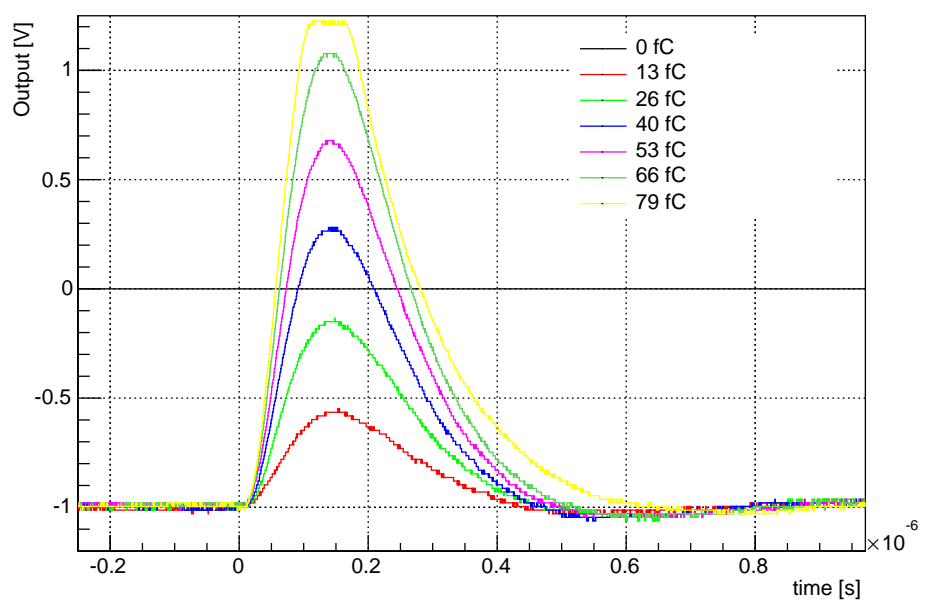

(c) Sen $=30 \mathrm{mV} / \mathrm{fC}$ (neg), $t_{s}=160 \mathrm{~ns}, Q_{i n}=0 \mathrm{C}$ to $79 \mathrm{fC}, \mathrm{Cd}=20 \mathrm{pF}$.

Figure 5.7: Measured output pulses for different SAMPA chip01 configurations 


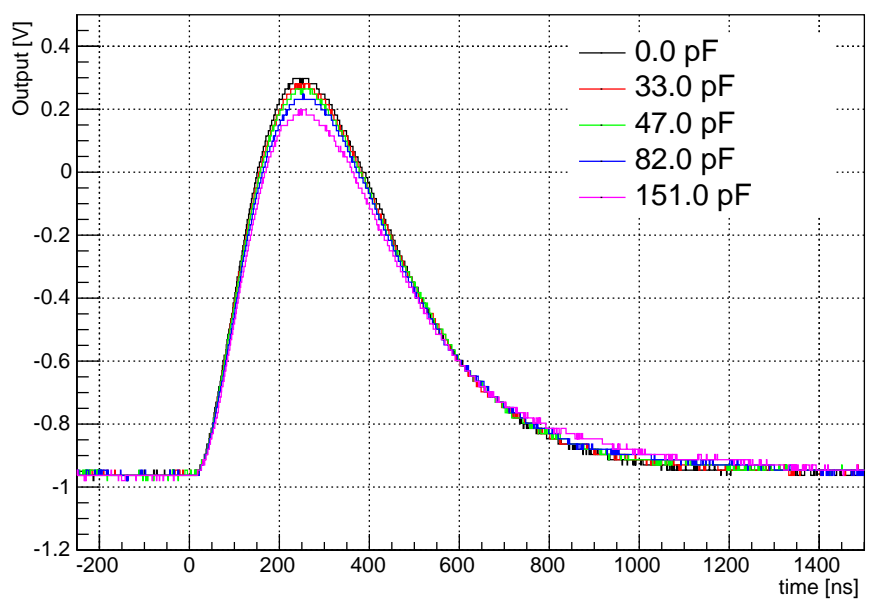

(a) Sen $=4 \mathrm{mV} / \mathrm{fC}, t_{s}=300 \mathrm{~ns}$.

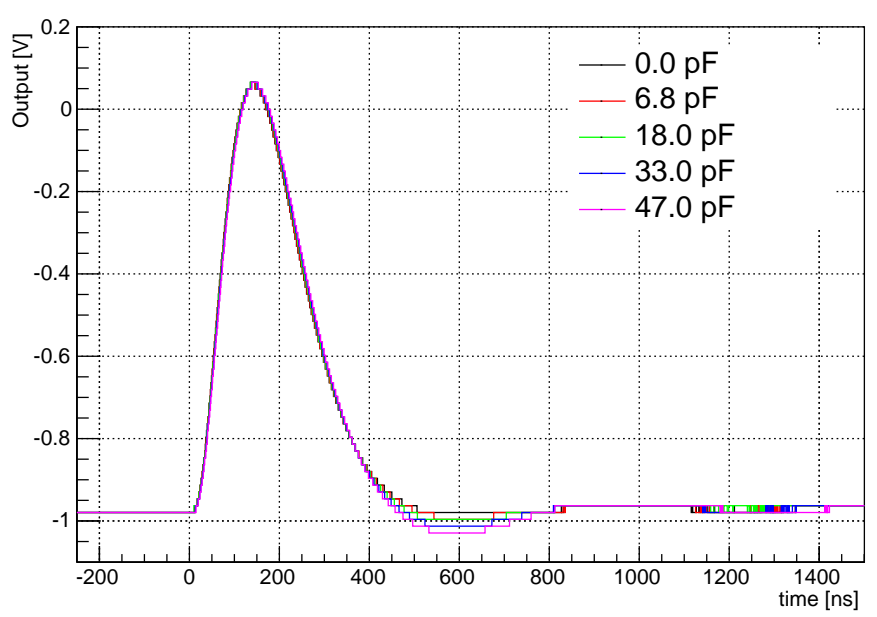

(b) Sen $=20 \mathrm{mV} / \mathrm{fC}, t_{s}=160 \mathrm{~ns}$.

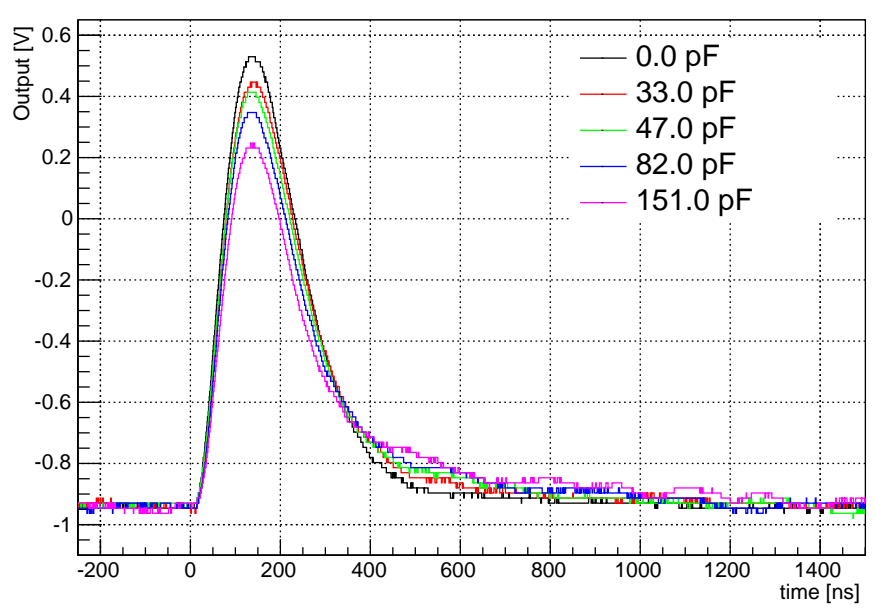

(c) Sen $=30 \mathrm{mV} / \mathrm{fC}, t_{s}=160 \mathrm{~ns}$.

Figure 5.8: Chip01 measured analog output signals for a range of detector capacitances. 


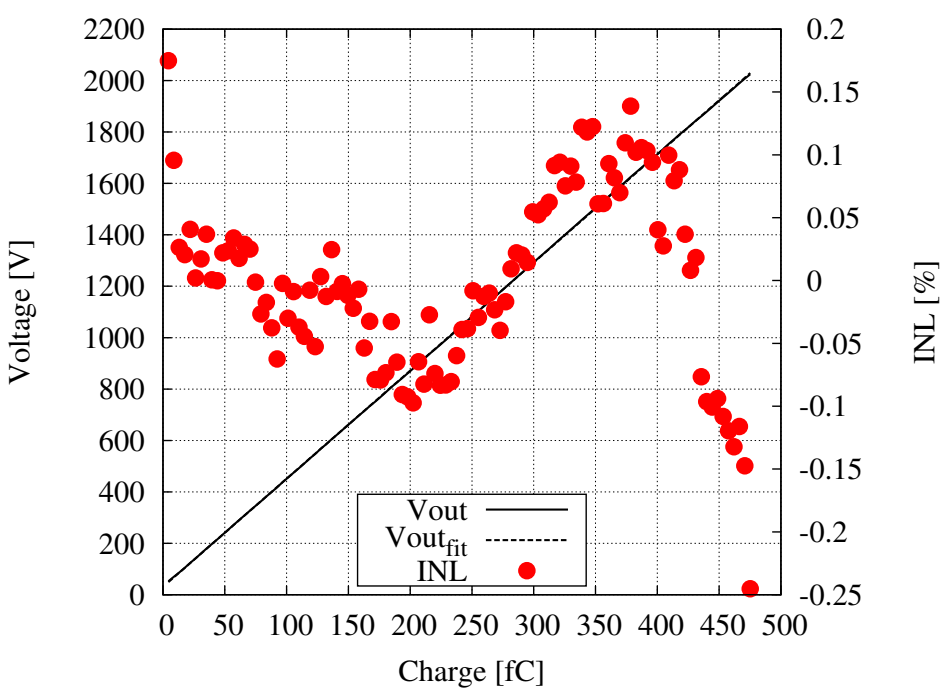

(a) $4 \mathrm{mV} / \mathrm{fC}$

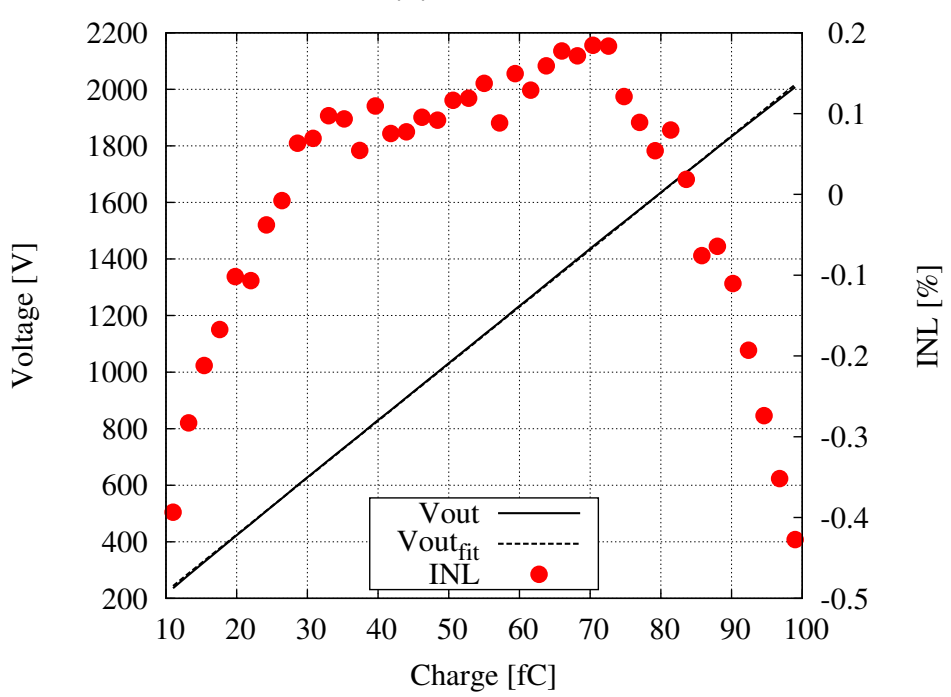

(b) $20 \mathrm{mV} / \mathrm{fC}$

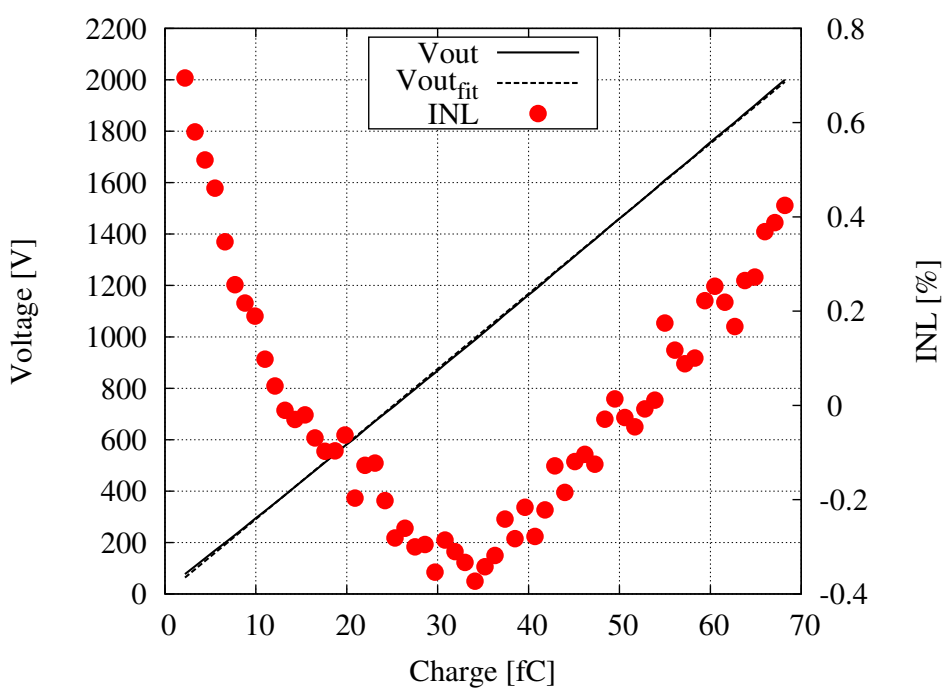

(c) $30 \mathrm{mV} / \mathrm{fC}$

Figure 5.9: Amplifier output peak voltage (Vout), linear fit $\left(\right.$ Vout $\left._{f i t}\right)$ and INL[\%] as function of the input charge for the 3 sensitivity cases. 


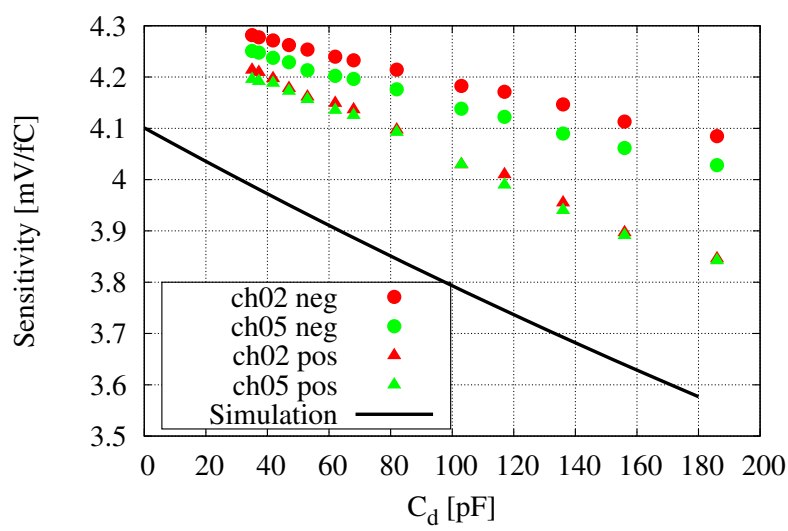

(a)

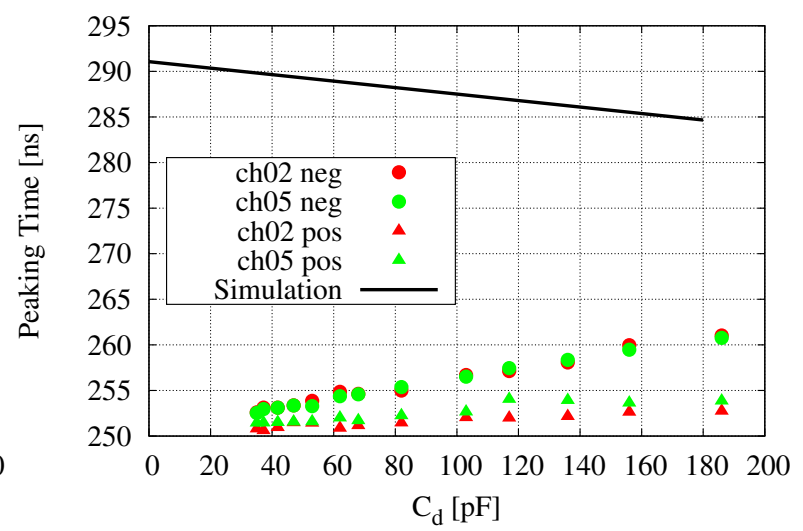

(b)

Figure 5.10: (a) Circuit sensitivity and (b) peaking time as a function of the detector capacitance for measurements (points) and simulation (straight line) for $4 \mathrm{mV} / \mathrm{fC}$ configuration.

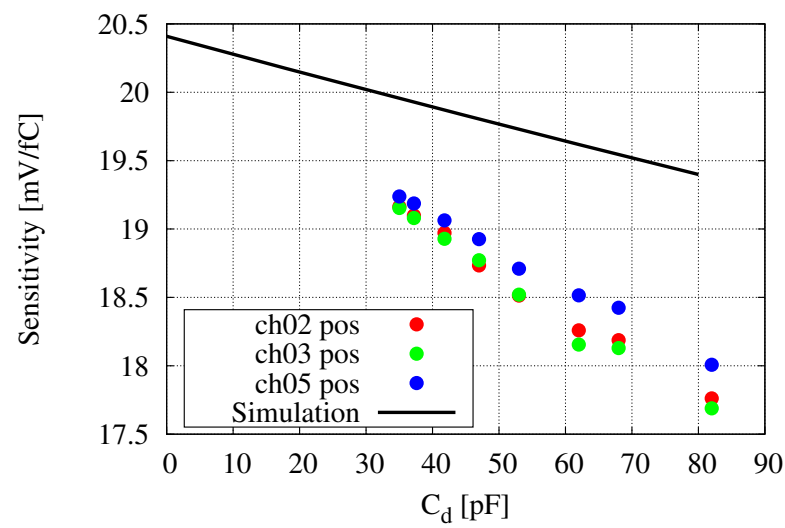

(a)

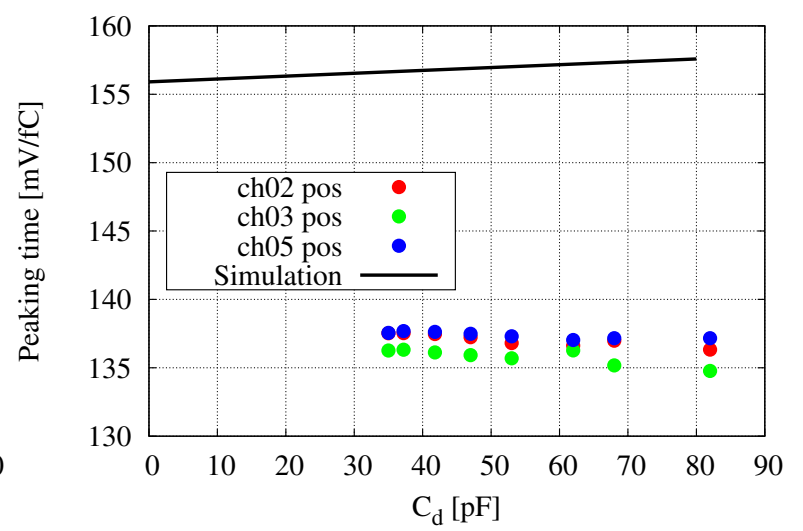

(b)

Figure 5.11: (a) Circuit sensitivity and (b) peaking time as a function of the detector capacitance for measurements (points) and simulation (straight line) for $20 \mathrm{mV} / \mathrm{fC}$ configuration.

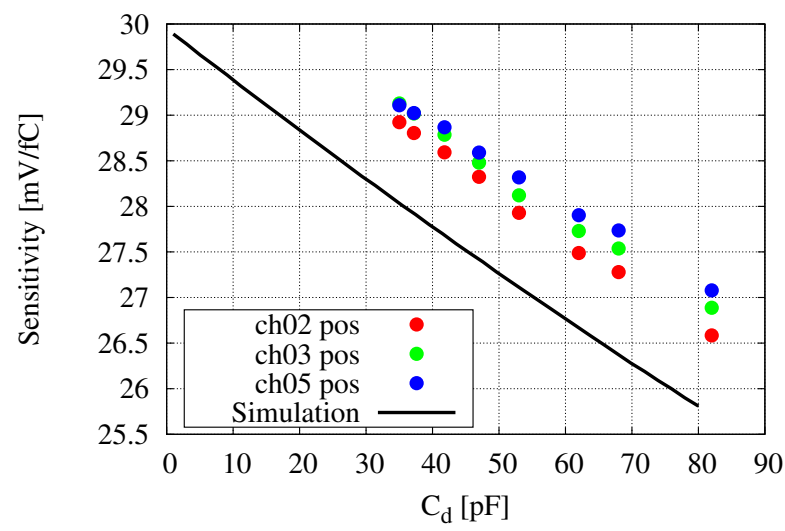

(a)

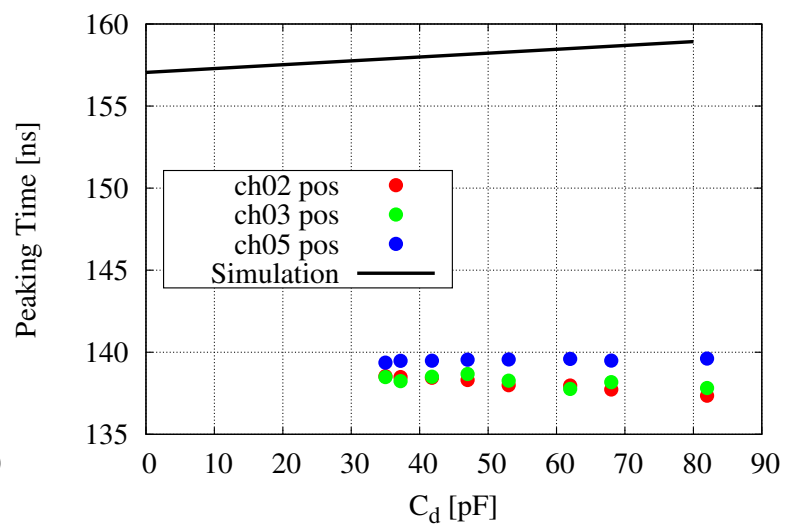

(b)

Figure 5.12: (a) Circuit sensitivity and (b) peaking time as a function of the detector capacitance for measurements (points) and simulation (straight line) for $30 \mathrm{mV} / \mathrm{fC}$ configuration. 


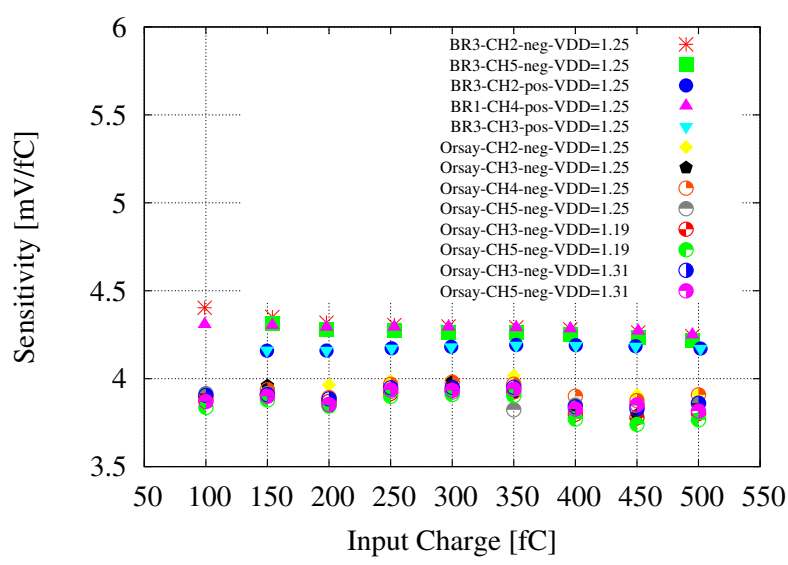

(a)

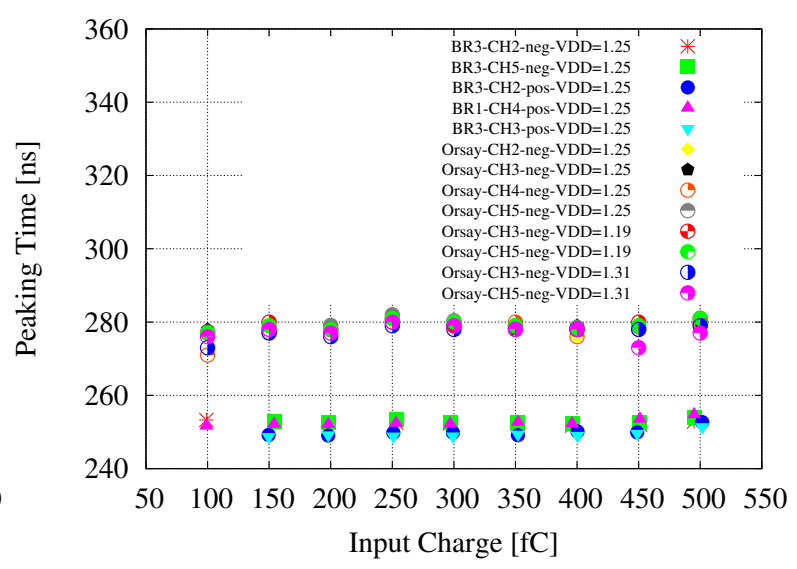

(b)

Figure 5.13: (a) Circuit sensitivity and (b) peaking time measures as a function of the input charge for the $4 \mathrm{mV} / \mathrm{fC}$ configuration.

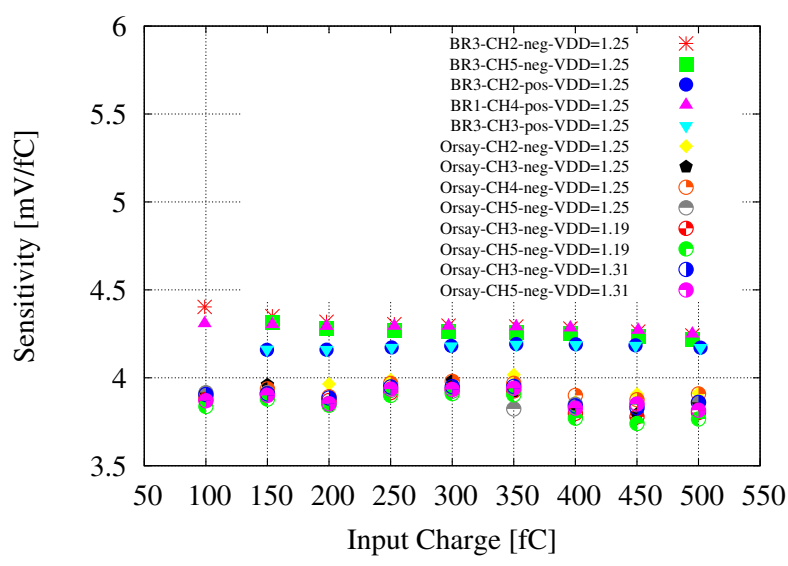

(a)

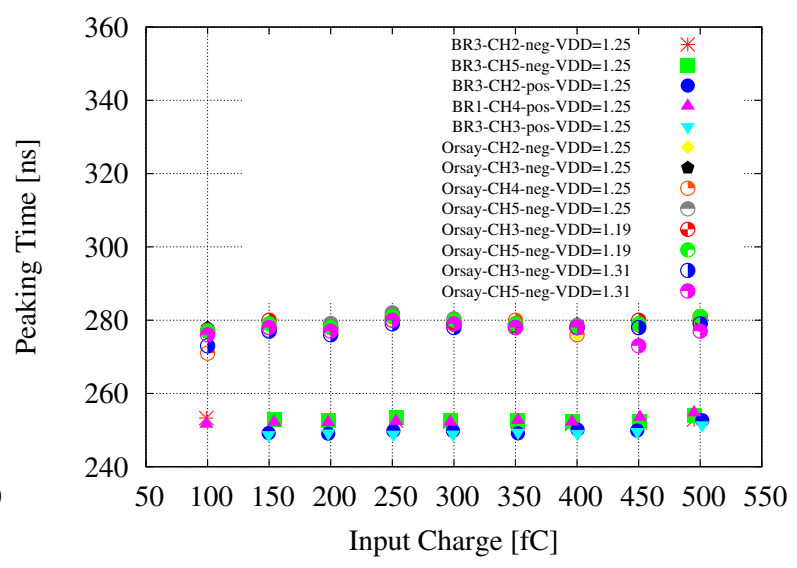

(b)

Figure 5.14: (a) Circuit sensitivity and (b) peaking time measures as a function of the input charge for the $20 \mathrm{mV} / \mathrm{fC}$ configuration.

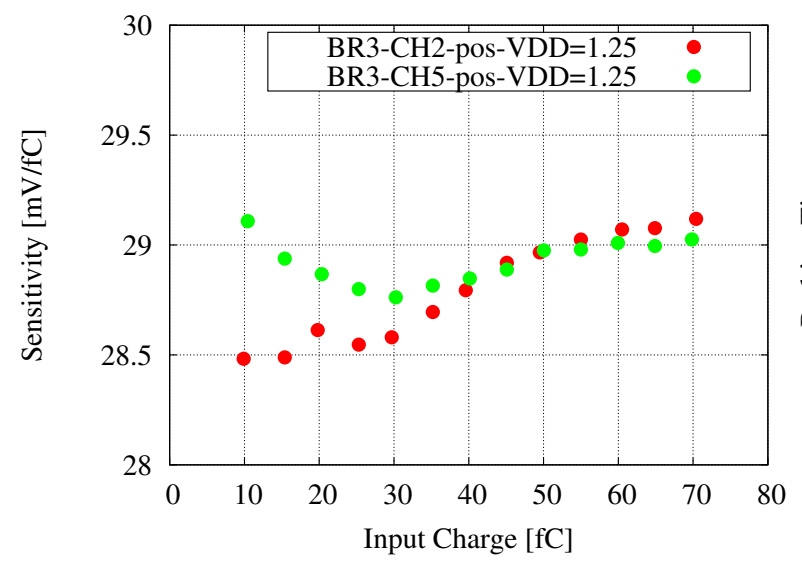

(a)

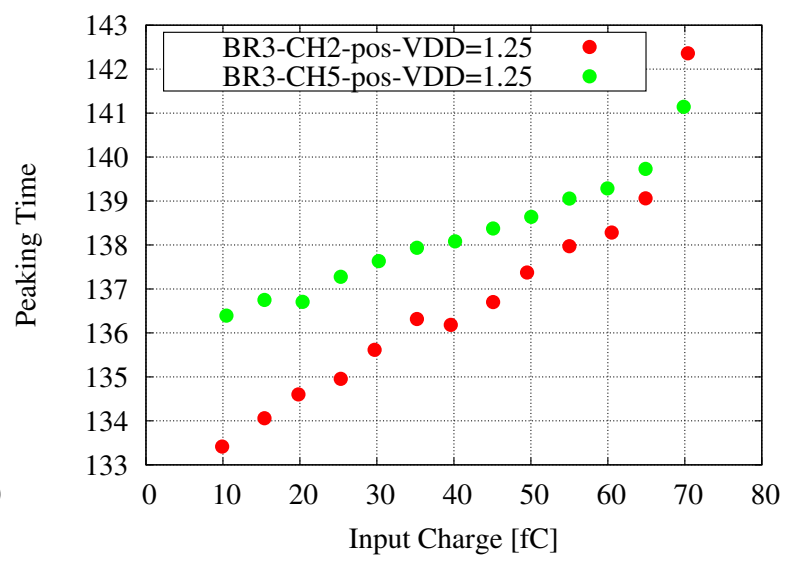

(b)

Figure 5.15: (a) Circuit sensitivity and (b) peaking time measures as a function of the input charge for the $30 \mathrm{mV} / \mathrm{fC}$ configuration. 


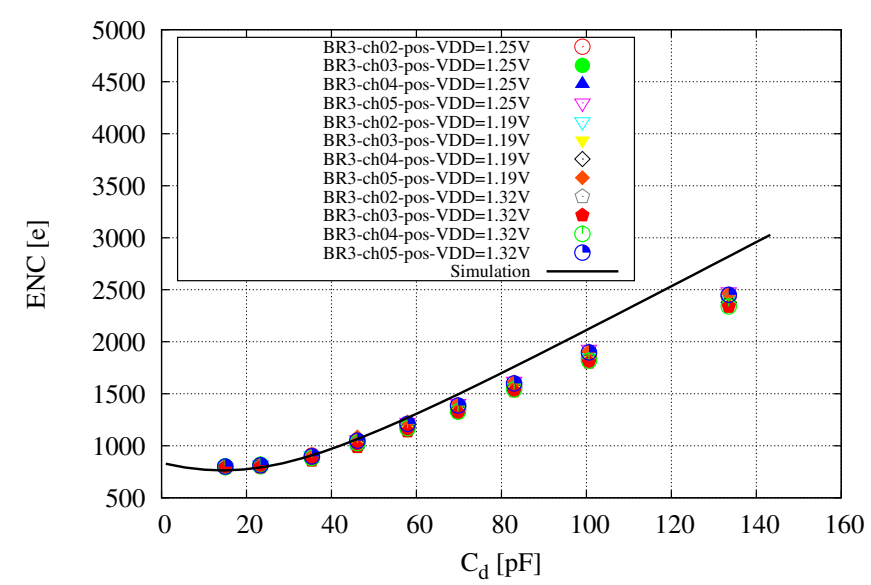

Figure 5.16: Measured equivalent noise charge of the SAMPA chip01. The points show the measurements done for various chips and straight line the expected value from simulation for the $4 \mathrm{mV} / \mathrm{fC}$ configuration.

\subsubsection{Noise (ENC)}

The RMS output noise $V_{R M S}$ of the produced chip was measured using the AC RMS value of the differential output voltage from the digital oscilloscope $\left(V_{R M S}\right)$ within of a $10 \mu \mathrm{s}$ time window. The measured values of $V_{R M S}$ and sensitivity were used to estimated the ENC specification according to the equation:

$$
E N C=\frac{V_{R M S}}{q \cdot \text { Sensitivity }}
$$

The system set-up for this test was equal to the used in the output pulse characterization test (see Figure 5.5). The $V_{R M S}$ was measured after taking out the charge injection cable (front-end channel is not pulsing). These measurements were done for each peaking time and sensitivity configurations of the chip, also for the power supply and detector capacitance corners. The results are shown in Figures 5.16, 5.17 and 5.18 together with the simulated values for each configuration indicating the chip name, tested channel, the polarity and the voltage supply. The measured equivalent noise charge was $850 \mathrm{e}$ for $4 \mathrm{mV} / \mathrm{fC}$ at an input capacitance of $40 \mathrm{pF}, 550 \mathrm{e}$ for $20 \mathrm{mV} / \mathrm{fC}$ at an input capacitance of $18.5 \mathrm{pF}$ and $550 \mathrm{e}$ for $30 \mathrm{mV} / \mathrm{fC}$ at an input capacitance of $18.5 \mathrm{pF}$, which is quite difference from the specification of the experiment. The noise measurements indicate a discrepancy between the measurements and the simulated values based on the models for TCSMC $0.13 \mu \mathrm{m}$ technology. 


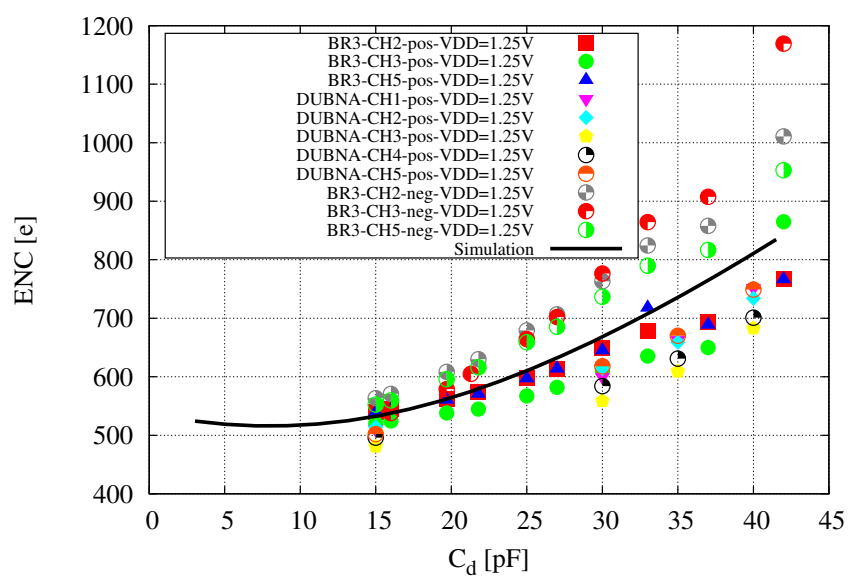

Figure 5.17: Measured equivalent noise charge of the SAMPA chip01. The points show the measurements done for various chips and straight line the expected value from simulation for the $20 \mathrm{mV} / \mathrm{fC}$ configuration.

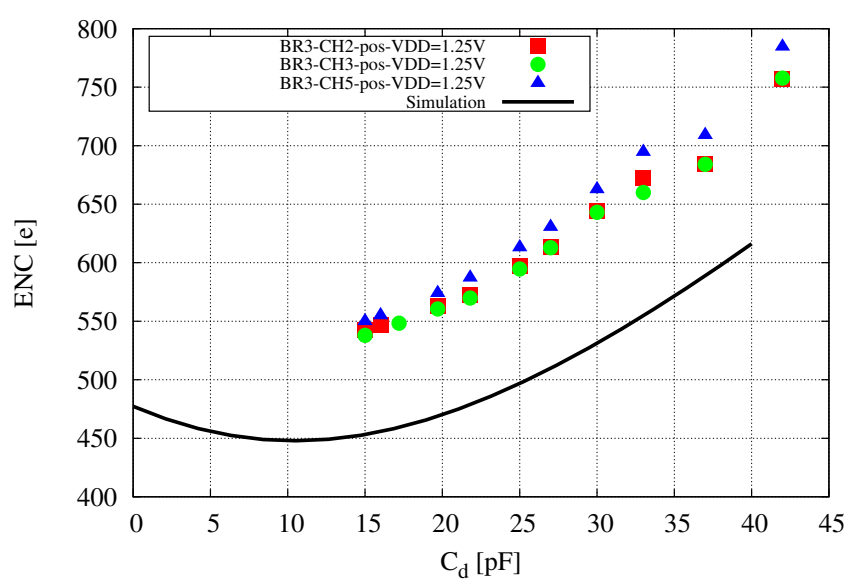

Figure 5.18: Measured equivalent noise charge of the SAMPA chip01. The points show the measurements done for various chips and straight line the expected value from simulation for $30 \mathrm{mV} / \mathrm{fC}$ configuration.

\subsubsection{Cross-talk}

The Cross-talk specification is defined by signals coupling between adjacent channels due to the package and board parasitic capacitance. To obtain this specification a full range input charge is injected on a determined channel and the amplitude of the output pulse of each channel is measured. The above procedure was done in the same way for each channel and for each chip configuration options with the typical detector capacitance. The Cross-talk can be calculated from the acquired data by the following equation:

$$
\text { Crosstalk }=\frac{V_{A D J}}{V_{C H}} \times 100
$$

where $V_{A D J}$ is the pulse amplitude of the adjacent channel and $V_{C H}$ is the pulse 


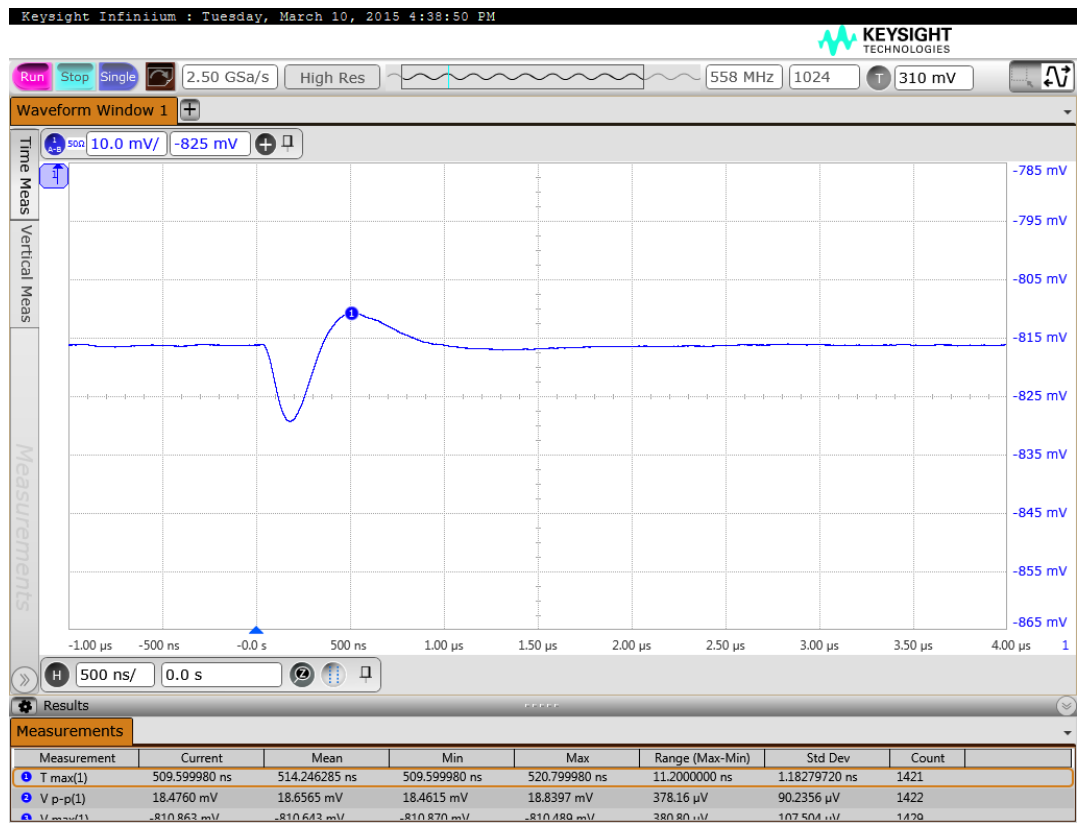

Figure 5.19: Experimental output parasitic pulse detected on channel 5 when channel 4 is pulsing

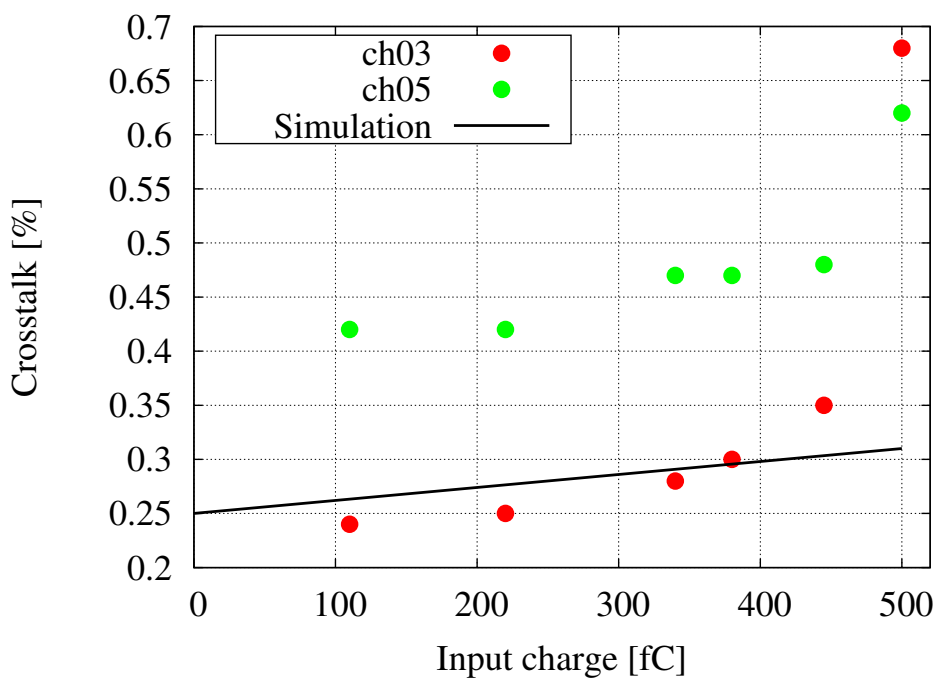

Figure 5.20: Experimental crosstalk specification on channel 5 and 3 in terms of the input charge when channel 4 is pulsing, for the Sen $=30 \mathrm{mV} / \mathrm{fC} @ t_{s}=160 \mathrm{~ns}$ (a) and Sen=4mV/fC@ $t_{s}=300 \mathrm{~ns}(\mathrm{~b})$ cases.

amplitude of the injected channel. The specification obtained from crosstalk testing was higher than $0.8 \%$ which is out of the system specification $(<0.3 \%)$. Extra crosstalk component can be generated by the common inductance between of the bond-wire and the low Power Supply Rejection Ratio of the front-end at $\mathrm{MHz}$ frequencies. The design should be evaluated with more detail to avoid these extra crosstalk components in the next prototype run. Figure 5.19 shows the experimental output parasitic pulse detected on channel 5 when channel 4 is pulsing. A crosstalk measurement sample is presented in Figure 5.20 where the experimental crosstalk specification on channel 5 and 3 in 
terms of the input charge when channel 4 is pulsing, for the $\mathrm{Sen}=30 \mathrm{mV} / \mathrm{fC} @ t_{s}=160 \mathrm{~ns}$ (a) and Sen=4mV/fC@ $t_{s}=300 \mathrm{~ns}$ (b) cases. The detector capacitance used in this measure was $20 \mathrm{pF}$.

\subsubsection{PSRR of the Front-end}

All the tests of the SAMPA chip01 were performed with the chip mounted in the test board developed for this purpose. The test board is the same for all the tests and was designed to get rid of (or at least strongly suppress) environmental perturbation and having the cleanest measurement possible. In particular, plenty of capacitors were present to filter the $V_{D D}$, and therefore reducing the influence of the power supply noise and ripple. This turn to be a problem in the moment we went for the PSRR referred to te ouputput measurement. The real perturbation at the chip VDD inputs was by far smaller than the signal injected in the power supply of the board.

In order to perform the PSRR to te ouputput measure the power supply was replaced with a signal generator. The signal generator was configured for a load impedance of about $33 \Omega$. The signal generator was set to provide a sinusoidal signal, with $75 \mathrm{mVpp}$ amplitude, with offset adjusted so to provide the nominal 1.25V DC at the board power connector. The signal amplitude on $V_{D D}$ was measured via a single ended probe, therefore measuring it in one of the closest accessible point.

The front-end output of the chip was measured via a differential probe. Both the differential (chip output) and single ended (on $V_{D D}$ ) probes were connected to the same high performance, native 10 bits, oscilloscope. A FFT, via the oscilloscope function, was performed for both signals. The amplitude of the peak at the stimulation frequency was measured, averaging the value over about 1000 samples. A total of 26 measurements were done, at frequency values logarithmic spaced in the range $1 \mathrm{kHz}$ - $10 \mathrm{MHz}$. The PSRR to te ouputput measurements of a chip sample were performed for $20 \mathrm{mV} / \mathrm{fC}$ and $30 \mathrm{mV} / \mathrm{fC}$ configurations and they are compared with the simulation results in Figure 5.21 for $18.5 \mathrm{pF}$ of detector capacitance. A PSRR around $4 \mathrm{~dB}$ was obtained for $30 \mathrm{mV} / \mathrm{fC}$ configuration and $7 \mathrm{~dB}$ was obtained for $20 \mathrm{mV} / \mathrm{fC}$. 


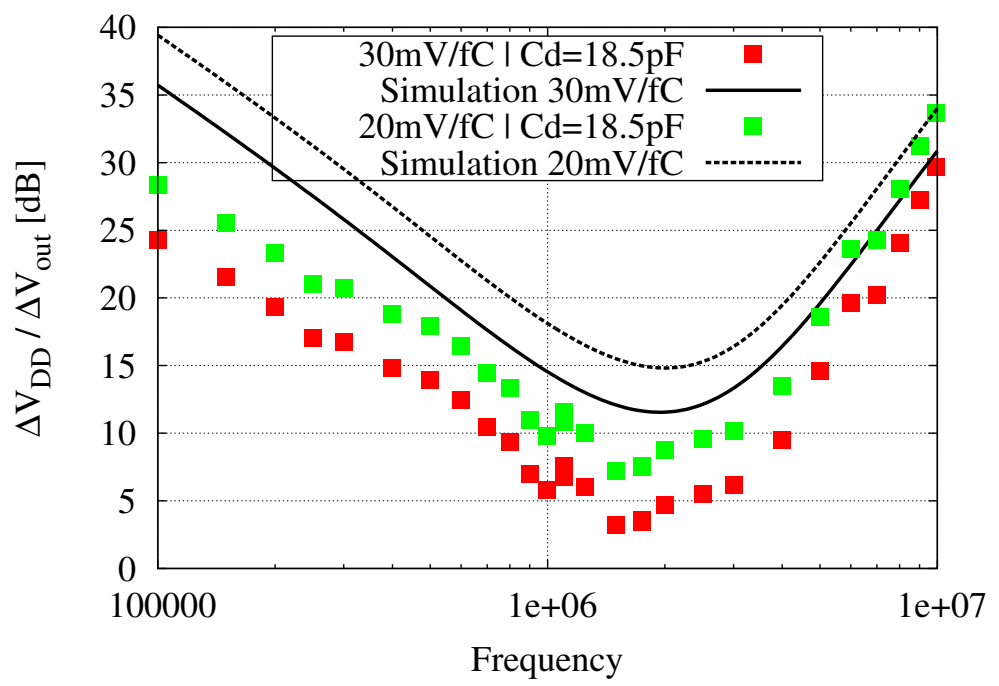

Figure 5.21: PSRR measurements for $20 \mathrm{mV} / \mathrm{fC}$ and $30 \mathrm{mV} / \mathrm{fC}$ configurations compared with the simulations results. 


\section{Chapter 6}

\section{Conclusions and Future Work}

There are in the literature many works about readout front-end design for high energy experiments, where the noise optimization during the CSA design have been approached. These works have only considered the channel thermal noise and the flicker noise of the CSA input transistor as the more important contributor. It is considering that $90 \%$ of the total noise specification is due to these components, and other noise source, as the noise generated by the bias current mirror, have been ignored.

The noise components generated by the bias current mirror transistor have an important impact on the total noise specification for low voltage high dynamic range frontends applications. A method to reduce these noise components is the introduction of a gate-source decoupling capacitance on the current mirror transistors which will perform a Low Pass Filter function in combination with the transconductance of the transistor as diode. The dominant pole of this LPF combination must be placed at frequencies lower than the frequency band of the Semi-Gaussian Pulse Shaper. For front-ends with peaking time in the order of hundreds of ns, the decoupling capacitor is in the order of hundreds of $\mathrm{pF}$ as demonstrated in this work. In multichannel readout ASICs applications the area consumption to integrated these capacitor limits the number of channels per chip. The decoupling capacitor size can be reduced if the bias current by the transistor as diode of the current mirror is increase, but it will increase the CSA power consumption significantly.

New techniques to cancel/reduce the noise components from the bias current network and to improve the PSRR of the CSA amplifier have been studied in this thesis. The proposed topologies are based on the traditional cascode topology with NMOS in- 
put transistor. The first technique named of PMOS-NMOS input transistor topology was proposed in Chapter 4. It uses a PMOS transistor with the gate connected to the CSA input to generated a zero noise condition for a specific values of detector capacitance. The zero noise condition depend of the relation between the NMOS and PMOS inputs transistor transconductance and of the relation between the detector capacitance and the $C_{g s}$ capacitance of the proposed PMOS input transistor, which can be adjusted for the typical values of detector capacitance. A important result obtained with the PMOSNMOS input transistor topology is a lower variation than the total ENC specification in relation to the detector capacitance. The detector capacitance which is defined by the detector pad capacitance and the interconnection capacitance can vary from channel to channel of the gaseous detector. Low ENC increases when $C_{d}$ increase is desired because the detector pad size can be increased without losing noise performance.

The zero bias noise condition demands a careful design to match the input transistor transconductance relation and the gate-source capacitance of the PMOS input transistor. Accordingly, another topology was proposed in this work in which the zero bias noise condition does not depend of $C_{g s 2}$ and a more flexible design is possible. It might be imagined that the proposed topology is more sensitive to the process variation than the traditional NMOS input transistor topology, because the zero bias noise condition depends on process parameters. Corners simulation results show that the ENC variation of the proposed topologies are acceptable compared with the traditional topology.

The low PSRR at at the frequency band of the Semi-Gaussian shaper of the CSA is another limitation for low noise circuit operation. An important noise component from the power supply will be amplified by the pulse shaper to the front-end output if the CSA PSRR does not attenuate its. Furthermore, important crosstalk components will be generated if the the transient voltage drops of the supply voltage are not compensated by the CSA. In this thesis a PSRR analysis of the CSA with the traditional NMOS input transistor topology and the PSRR improvement obtained with the proposed topologies input transistor topology was presented in Chapter 4. A comparison of the PSRR at $2 \mathrm{MHz}$ of the designed CSA with the NMOS input transistor topology $\left(P S R R_{N M O S}\right)$, with the PMOS-NMOS input transistor topology and with the $C_{r}$ feedback topology $\left(P S R R_{C_{r}}\right)$ for $t_{s}=160 \mathrm{~ns}$ and $S e n=30 \mathrm{mV} / \mathrm{fC}$ shows a PSRR improvement 
of $16 \mathrm{~dB}$, which was achieved with the proposed topologies for $18.5 \mathrm{pF}$ of detector capacitance.

A chip of the designed front-end was fabricated in 130nm TSMC CMOS technology with 6 Metal layers (the top metal is thick metal) via IMEC called the SAMPA chip01. The produced prototype contains 5 channels of the front-end block with independent inputs/outputs per channel and common control signals. The experimental results presented in Chapter 5 showed good, but them evinced a likely metallization mistake of the MIM capacitor used for the produced chip design. The linearity, Sensitivity and ENC measures exhibited a good matching with the simulation results, but the peaking time measures showed a significant difference if compared with the simulations. This difference was attributed to a metallization mistake. The pulse waveform for the negative polarity of the $160 \mathrm{~ns}$ cases presented a small undershoot which is undesirable for the TPC electronic system. This pulse undershoot was generated by a feedback effect from the pulse shaper to the CSA through the bias network. This effect was reproduced after more accurate post-layout simulations.

The techniques for bias noise cancellation presented in this thesis can be applied to others circuits as capacitive amplifiers for MEMs and neural amplifiers where noise is a critical specification. A propose for future works is explored new topologies for these circuits using the NMOS-PMOS input transistor configuration and the $C_{r}$ feedback topology to improve the PSRR at the $\mathrm{MHz}$ frequency band. Furthermore, new CSA topologies can be studied considering the noise cancellation of the input transistor which is the main noise contribution to the total noise specification. 


\section{Bibliography}

ALICE, Collaboration The. Upgrade of the ALICE Inner Tracking System. Geneva, 2013.

ALME, J. et al. The ALICE TPC, a large 3-dimensional tracking device with fast readout for ultra-high multiplicity events. Nuclear Instruments and Methods in Physics Research Section A: Accelerators, Spectrometers, Detectors and Associated Equipment, v. 622, n. 1, p. 316-367, 2010.

ASPELL, P. et al. Super-Altro 16: a Front-End System on Chip for DSP Based Readout of Gaseous Detectors. Feb 2013.

BLUM, Walter; RIEGLER, Werner; ROLANDI, Luigi. Particle detection with drift chambers; 2nd ed. Berlin: Springer, 2008.

BLUM, Werner Riegler Walter; ROLANDI, Luigi. Particle Detection with Drift Chambers. [S.I.]: Springer, 2008.

BOSCH, R.E. et al. The ALTRO chip: a 16-channel A/D converter and digital processor for gas detectors. In: Nuclear Science Symposium Conference Record, 2002 IEEE. [S.I.: s.n.], 2002. v. 1.

CERN Gas Detector Development group. Disponível em: <gdd.web.cern.ch/GDD>.

CHONG, Z.Y.; SANSEN, W.M.C. Low-Noise Wide-Band Amplifiers in Bipolar and CMOS Technologies. [S.I.]: Springer, 1991. (Kluwer international series in engineering and computer science: VLSI, computer architecture, and digital signal processing).

COLLABORATION, ALICE et al. ALICE: Physics Performance Report, Volume I. Journal of Physics G: Nuclear and Particle Physics, v. 30, n. 11, p. 1517, 2004.

COLLABORATION, The ALICE. ALICE Technical Design Report of the Time Projection Chamber. December 2013.

ENZ, Christian C.; KRUMMENACHER, François; VITTOZ, Eric A. An Analytical MOS Transistor Model Valid in All Regions of Operation and Dedicated to Low-voltage and Low-current Applications. Analog Integrated Circuits and Signal Processing, Kluwer Academic Publishers, Hingham, MA, USA, v. 8, n. 1, p. 83-114, jul. 1995.

GASPARI, Massimiliano De. System on Chip for High Energy Physics. Tese - Heidelberg University, Ruperto Carola, July 2012.

GRAMEGNA, G. et al. "\{CMOS\} preamplifier for low-capacitance detectors ". Nuclear Instruments and Methods in Physics Research Section A: Accelerators, Spectrometers, Detectors and Associated Equipment, v. 390, n. 1-2, p. 241-250, 1997. 
INIEWSKI, Krzysztof. Electronics for Radiation Detection. [S.I.]: Taylor \& Francis Group, 2010.

JOHNS, D.A.; MARTIN, K. Analog Integrated Circuit Design. [S.I.]: Wiley India Pvt. Limited, 2008.

KARLEN, Dean et al. "investigation of gem space point resolution for a tpc tracker". v. 578 , p. 817-820, 2001.

LEE, Dongwoo; BLAAUW, D.; SYLVESTER, D. Gate oxide leakage current analysis and reduction for VLSI circuits. Very Large Scale Integration (VLSI) Systems, IEEE Transactions on, v. 12, n. 2, p. 155-166, Feb 2004.

LIN, M.B. Introduction to VLSI Systems: A Logic, Circuit, and System Perspective. [S.I.]: Taylor \& Francis, 2011.

LOINAZ, Marc J.; WOOLEY, Bruce A. A CMOS Multichannel IC for Pulse Timing Measurements with 1-mV Sensitivity. IEEE Journal Solid-State Circuits, v. 30, n. 12, p. 1339-1340, December 1995.

MUSA, Luciano. The time projection chamber for the ALICE experiment . Nuclear Physics A, v. 715, n. 0, p. 843c - 848c, 2003. Quark Matter 2002 Proceedings of the 16th International Conference on Ultra-Relativistic Nucleus-Nucleus Collisions.

MUSA, Luciano. The ALICE TPC Front End Electronics in short. 2007. Disponível em: <http://ep-ed-alice-tpc.web.cern.ch/ep-ed-alice-tpc/>.

PROSPECTS for charge sensitive amplifiers in scaled \{CMOS\}. Nuclear Instruments and Methods in Physics Research Section A: Accelerators, Spectrometers, Detectors and Associated Equipment, v. 480, n. 2-3, p. 713 - 725, 2002.

SANSEN, Willy; CHANG, Z-Y. Feedforward compensation techniques for highfrequency cmos amplifiers. Solid-State Circuits, IEEE Journal of, v. 25, n. 6, p. 15901595, Dec 1990.

SANSEN, W.; CHANG, Zhong-Yuan. Limits of low noise performance of detector readout front ends in CMOS technology. Circuits and Systems, IEEE Transactions on, v. 37, n. 11, p. 1375-1382, Nov 1990.

SAULI, Fabio. GEM: a new concept for electron amplification in gas detectors. Nuclear Instruments and Methods in Physics Research A386, p. 531, 1997.

SOLTVEIT, H K et al. The PreAmplifier ShAper for the ALICE TPC-Detector. Nucl. Instrum. Methods Phys. Res., A, v. 676, n. arXiv:1203.3564, p. 106-119. 29 p, Mar 2012.

SPIELER, Helmuth. Analog and Digital Electronics for Detectors. "Proceedings of the 2003 ICFA School on Instrumentation, Itacuruca, Brazil", 2003.

SPIELER, Helmuth. Semiconductor Detector Systems. [S.I.]: Oxford University Press, 2005. (Series on semiconductor science and technology). ISBN 9780191713248. 
SRINIVASAN, V.S. Senthil; PANDYA, Arun. Dosimetry aspects of hafnium oxide metaloxide-semiconductor (mos) capacitor. Thin Solid Films, v. 520, n. 1, p. $574-577$, 2011. ISSN 0040-6090. Disponível em: <http://www.sciencedirect.com/science/article/ pii/S0040609011013976>.

STEYAERT, M.S.J.; SANSEN, W. Power supply rejection ratio in operational transconductance amplifiers. Circuits and Systems, IEEE Transactions on, v. 37, n. 9, p. 1077-1084, Sep 1990.

TSIVIDIS, Yannis. Operation and Modeling of the MOS Transistor. New York, NY, USA: McGraw-Hill, Inc., 1987. 